\title{
Republic of Moldova: Request for a Three-Year Arrangement Under the Extended Credit Facility and Request for an Extended Arrangement
}

The following documents have been released and are included in this package:

- $\quad$ The staff report, prepared by a staff team of the IMF, following discussions that ended on October 28, 2009, with the officials of the Republic of Moldova on economic developments and policies. Based on information available at the time of these discussions, the staff report was completed on January 15, 2010. The views expressed in the staff report are those of the staff team and do not necessarily reflect the views of the Executive Board of the IMF.

- $\quad$ A supplement to the staff report—Debt Sustainability Analysis

- $\quad$ A staff statement.

- $\quad$ A Press Release.

- $\quad$ A statement by the Executive Director for the Republic of Moldova.

The document(s) listed below will be separately released.

Letter of Intent sent to the IMF by the authorities of the Republic of Moldova*

Memorandum of Economic and Financial Policies by the authorities of the Republic of Moldova *

Technical Memorandum of Understanding*

*Also included in Staff Report

The policy of publication of staff reports and other documents allows for the deletion of market-sensitive information.

\author{
Copies of this report are available to the public from \\ International Monetary Fund • Publication Services \\ $70019^{\text {th }}$ Street, N.W. • Washington, D.C. 20431 \\ Telephone: (202) 623-7430 • Telefax: (202) 623-7201 \\ E-mail: publications@imf.org Internet: http://www.imf.org
}

\section{International Monetary Fund Washington, D.C.}


INTERNATIONAL MONETARY FUND

\title{
REPUBLIC OF MOLDOVA \\ Request for a Three-Year Arrangement Under the Extended Credit Facility and Request for an Extended Arrangement
}

\author{
Prepared by the European Department \\ (In consultation with other departments) \\ Approved by Adam Bennett and Christian Mumssen
}

January 15, 2010

\section{Executive Summary}

- Fund Arrangements. In the attached letter, the Moldovan authorities are requesting combined Extended Credit Facility/Extended Fund Facility Arrangements each running for three years with access totaling SDR 369.6 million (300 percent of quota, or US\$588 million) split equally between the two facilities. An initial purchase of SDR 60 million becomes available upon approval of this request. The request is in support of the authorities' economic program, which aims to restore fiscal and external sustainability, preserve financial stability, and support growth. The program rests on four pillars: (i) fiscal policies to restore sustainability while safeguarding public investment and social spending priorities; (ii) flexible monetary and exchange rate policies to keep inflation under control, facilitate adjustment to shocks, and rebuild foreign reserves; (iii) policies to ensure financial stability by early detection of bank difficulties and strengthening the legal framework for bank rehabilitation and resolution; and (iv) structural reforms to raise the economy's potential.

- Discussions. During October 14-28, 2009, staff met with Prime Minister Filat, Deputy Prime Ministers Lazar and Osipov, Minister of Finance Negruta, First Deputy Governor of the National Bank of Moldova Cibotaru, Deputy Governors Tabirta and Molosag, Minister of State Bodiu, Minister of Labor, Social Protection, and Family Buliga, other senior officials, ambassadors, and representatives of the trade unions, banking, and business community. The mission also met with the leaders of the four parties in the ruling coalition. The mission held a press conference and issued a press statement.

- Staff. Mr. Gueorguiev (head), Messrs. Srour and Gorbanyov (EUR), Ms. Jurzyk (SPR), and Mr. Karam (FAD). Mr. Mathisen and Mr. Mirzoev, outgoing and incoming resident representatives, assisted the mission. The mission cooperated closely with World Bank staff on structural issues. Mr. Yotsov, Advisor to the Executive Director for Moldova, attended most meetings. 


\section{List of Acronyms}

\begin{tabular}{|c|c|}
\hline ANRE & National Agency for Energy Regulation \\
\hline CAR & Capital asset ratio \\
\hline CEDB & Council of Europe Development Bank \\
\hline CIRR & Commercial interest reference rates \\
\hline DGF & Deposit Guarantee Fund \\
\hline DSA & Debt sustainability analysis \\
\hline EBRD & European Bank for Reconstruction and Development \\
\hline EC & European Commission \\
\hline ECF & Extended Credit Facility \\
\hline EFF & Extended Fund Facility \\
\hline EGPRSP & Economic Growth and Poverty Reduction Strategy Paper \\
\hline EIB & European Investment Bank \\
\hline FAD & IMF Fiscal Affairs Department \\
\hline FDI & Foreign direct investment \\
\hline FX & Foreign exchange \\
\hline IFI & International financial institution \\
\hline GRA & General Resources Account \\
\hline JSAN & Joint Staff Advisory Note \\
\hline LGD & Deposit Guarantee Law \\
\hline LOI & Letter of Intent \\
\hline MDL & Moldovan leu \\
\hline MEFP & Memorandum on Economic and Financial Policies \\
\hline MoF & Ministry of Finance \\
\hline MoU & Memorandum of Understanding \\
\hline NDA & Net Domestic Assets \\
\hline NFA & Net Foreign Assets \\
\hline NIR & Net International Reserves \\
\hline NBM & National Bank of Moldova \\
\hline NBS & National Bureau of Statistics \\
\hline NDS & National Development Strategy \\
\hline NPL & Nonperforming loan \\
\hline OECD & Organisation for Economic Co-operation and Development \\
\hline PA & Prior action \\
\hline PV & Present value \\
\hline PPP & Purchasing power parity \\
\hline PRGF & Poverty Reduction and Growth Facility \\
\hline REER & Real effective exchange rate \\
\hline SDR & Special Drawing Rights \\
\hline TMU & Technical Memorandum of Understanding \\
\hline VAT & Value added tax \\
\hline
\end{tabular}


I. Background and Recent Challenges ................................................................4

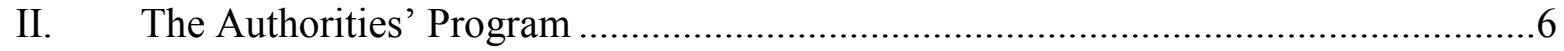

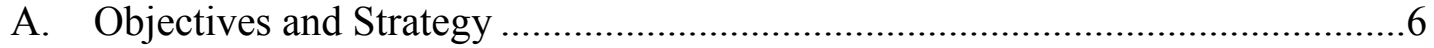

B. Macroeconomic Framework and Risks ........................................................ 7

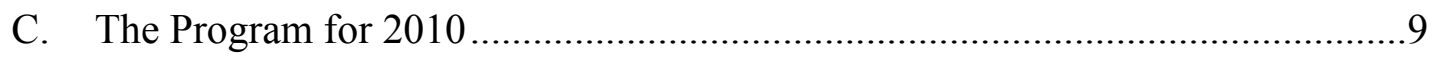

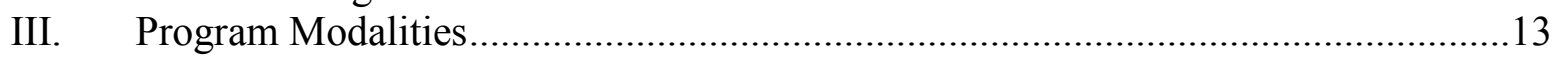

A. Access and Phasing ..............................................................................13

B. Capacity to Repay the Fund and Risks to the Program .................................14

C. Program Monitoring and Conditionality................................................. 14

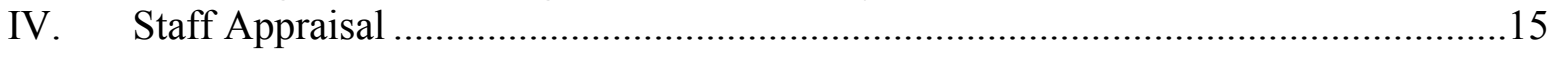

Figures

1. A Difficult Macroeconomic Situation ............................................................. 17

\section{Tables}

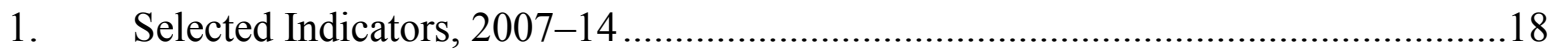

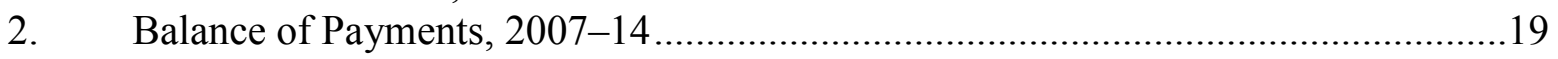

3. General Government Budget, 2007-14 ............................................................20

4. Accounts of the National Bank of Moldova and Monetary Survey, 2007-10..........22

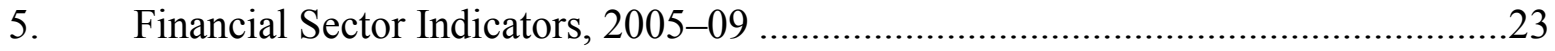

6. External Financing Requirements and Sources, 2009-12 .....................................24

7. Disbursements, Purchases, and Timing of Reviews Under the ECF/EFF

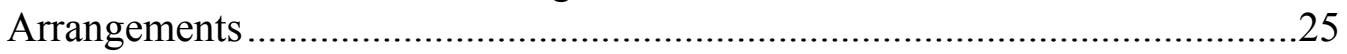

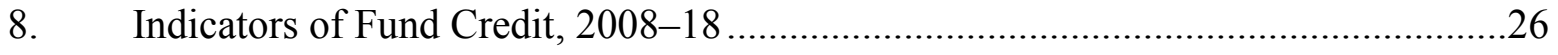

9. Localized Millennium Development Goals .....................................................27

Attachments

I. Letter of Intent, Memorandum of Economic and Financial Policies, and Technical Memorandum of Understanding .28 


\section{BACKGROUND AND RECENT CHALLENGES}

1. Moldova experienced strong growth over 2006-08, accompanied by signs of overheating. Growth averaged 5 percent, boosted by remittances and foreign investment. Buoyant domestic demand, however, pushed the current account deficit to 17 percent of GDP and generated inflation pressures. These pressures, together with large inflows, resulted in substantial appreciation of Moldova's real effective exchange rate (REER). Monetary policy kept real interest rates low despite double-digit inflation (Figure 1). Although fiscal policy maintained a small headline budget deficit under the IMF PRGF-supported program, it grew increasingly procyclical, with the estimated structural balance deteriorating from a small surplus in 2005 to a deficit of over 4 percent of GDP by 2008.

\section{The economy remained overregulated and hampered by relative price}

distortions. High barriers to entry and low competition in telecommunications, trade, and food processing kept domestic prices significantly above international prices of many consumer products. In contrast, utility tariffs generally remained well below cost-recovery levels, leading to substantial arrears and underinvestment.

3. The global economic crisis led to a sharp weakening of the economy. In the first half of 2009, falling demand in trading partners led to a severe downturn in exports and remittances. While GDP dropped by nearly 8 percent over the same period, domestic demand declined even faster, and the current account deficit contracted to about 11 percent of period GDP. At the same time, the balance of payments moved from a surplus to a large deficit as FDI and other capital inflows fell dramatically. Deflation pressures emerged, with the 12month inflation at -0.7 percent in November (Figure 1).

\section{Two rounds of parliamentary elections marked a rise in political instability} in 2009. The April elections were followed by strong protests against alleged irregularities and boycott of the parliament by the opposition parties. A repeat election held in July led to the formation of a new four-party coalition government with a narrow majority. However, political uncertainty persists as parliament failed to elect a President of the republic.

\section{The crisis and pre-election spending hikes resulted in a large increase in the} fiscal deficit. Over January-September 2009, budget revenue dropped by about 10 percent in real terms relative to 2008 as absorption-related tax receipts declined. At the same time, current fiscal expenditure increased by over 13 percent (also in real terms), driven by large increases in wages and pensions in the run-up to the April elections. The fiscal deficit increased from 1 percent of GDP in 2008 to 61/4 percent of GDP in January-September 2009, financed mainly by a drawdown of balances in budget accounts and heavy domestic borrowing. The budget began to experience financing shortfalls, and arrears started to accumulate. Policy slippages derailed the PRGF-supported program, which expired in May 2009 without completion of the last two reviews. 


\section{The authorities used most of their SDR allocation for budget financing in}

late 2009. The Ministry of Finance assumed an obligation of SDR 114.3 million to the members of the SDR department (out of a total allocation of SDR 117.7 million). Given the severe external financing shortages and the difficulties in adjusting inherited expenditure commitments in the short run, this operation reduced reliance on expensive short-term domestic financing and cleared accumulated expenditure arrears. The NBM's gross international reserves were not affected as the foreign exchange equivalent of the SDRs was sold to the central bank.

7. Monetary policy has been tight despite falling inflation. Resisting sustained depreciation pressures, the National Bank of Moldova (NBM) sold about a third of its international reserves in early 2009, sharply tightening liquidity despite rapidly falling inflation. Nevertheless, the REER depreciated notably in January-April 2009, and again in December (10 percent), offsetting in large part the appreciation seen in previous years. Since May, the NBM partially replenished its reserves, cut its base rate by 6 percentage points and lowered reserve requirements in half. Nonetheless, commercial banks' lending rates remain

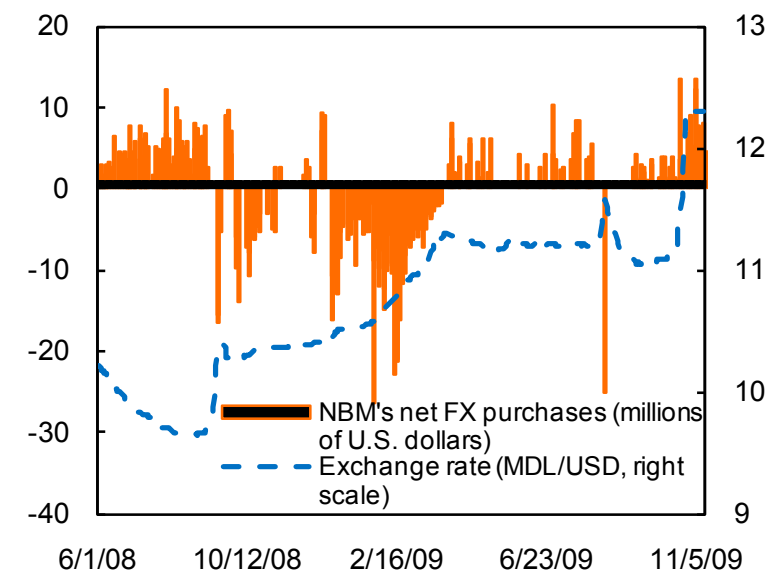
very high in real terms, contributing - together with the depressed economy - to a sharp drop in demand for credit (Figure 1). The supply of credit has also tightened, as banks channel the released liquidity into T-bills or keep it deposited with the NBM.

\section{While the financial system appears} stable, the recession is affecting credit quality, and one bank has been closed. The decline in credit and bank capital increases brought the capital adequacy ratio of the system to 32.7 percent in November 2009, well above the required minimum of 12 percent. Foreign banks have been maintaining exposure to their Moldovan subsidiaries, which account for 26 percent of banking system assets. Stress tests conducted by the NBM confirm that most banks' portfolios are robust to various risks. However, the nonperforming loan ratio

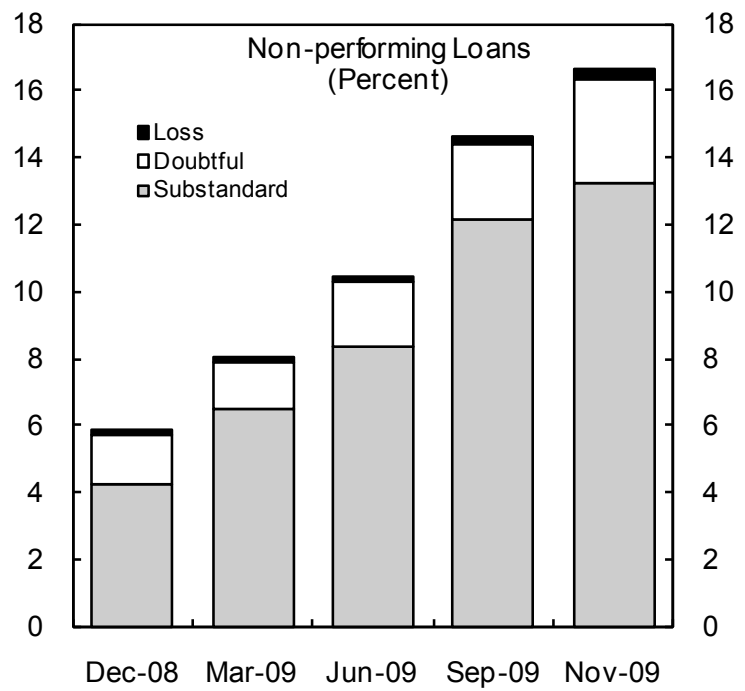
reached 16.6 percent in November 2009, and provisioning remains low. Moreover, one medium-size bank (Investprivatbank, IPB) became insolvent in June as a result of high 
portfolio concentration in recession-hit sectors and risk management irregularities. At the same time, low bank interconnectedness limits the systemic threat from individual bank failures. Moreover, the NBM has stepped up its supervision and regulation (MEFP $\mid 6$ ).

9. Difficulties in maintaining cost-recovery tariffs for district heating continue, creating a budget risk. The municipality of Chisinau has kept the district heating tariff at its 2007 level despite the significant cost increase since then. As a result, the heating company has been running large losses and accumulating arrears to its suppliers, exceeding 3 percent of GDP by late 2009. Nonpayment to the gas supply company risks a repeat of the 2008 cut-off in gas supplies to heat producers. The tension between the below-cost tariff and the need to ensure adequate payments to suppliers creates a risk for the consolidated budget, as it may be called upon for financing.

10. Debt is sustainable at present, but vulnerabilities are rising. The debt sustainability analysis (DSA) indicates that Moldova's risk of debt distress is low given presently envisaged borrowing, mainly from IFIs. However, alternative scenarios suggest that rapid accumulation of nonconcessional debt - faster than US\$125 million per year-can increase the risk of debt distress, warranting caution. Moreover, high private external debt for a developing country (44 percent of GDP) also signals heightened vulnerabilities.

\section{The Authorities' Program}

\section{A. Objectives and Strategy}

11. The program aims to restore fiscal and external sustainability, preserve financial stability, and raise growth. Specifically, program objectives include: (i) reversing the structural fiscal deterioration that occurred in 2008-2009 while safeguarding funds for public investment and priority social spending; (ii) keeping inflation under control while rebuilding foreign reserves to cushion the economy from external shocks; (iii) ensuring financial stability by enabling early detection of problems and strengthening the framework for bank rehabilitation and resolution; and (iv) raising the economy's potential through structural reforms.

12. The authorities are embarking on a path to restore fiscal sustainability by 2012. They target a structural budget deficit adjustment of about 6 percentage points of GDP, steadily reducing the headline deficit from 9 percent of GDP in 2009 to 7 percent in 2010, 5 percent in 2011, and 3 percent by 2012. This fiscal effort should bring the budget back to sustainability at a speed matching the economy's recovery. It will be achieved mainly through reform-based reduction of current expenditure to affordable levels, while public investment will be raised and the social safety net enhanced. In addition, raising revenue from consumption-based taxes, reinstating the corporate income tax in the medium term, and modernizing tax administration should support the adjustment. 
13. The NBM is strengthening its monetary policy framework around the objective of price stability. The NBM has approved a strategy clarifying the objectives, instruments, and means of communication of monetary policy. The strategy upholds price stability as the primary objective of monetary policy, operationalized as an annual inflation target, and the NBM's base rate as the main policy instrument. The clarity brought by the strategy is expected to help lower the large differential between the NBM policy rate and bank lending and deposit rates. A relatively high degree of financial dollarization, however, limits monetary policy effectiveness. Going forward, promoting de-dollarization and domestic currency debt markets will help strengthen the interest rate channel of monetary policy transmission.

\section{Structural reforms will aim to improve the business environment and support}

the fiscal effort. Over the whole program period, a massive program of deregulation and liberalization will free business initiative, stimulate competition, and strongly support the economic recovery. In 2010, actions in the district heating sector will address the acute fiscal risk stemming from prices set below cost. Reforms in the social insurance system aim to keep it sustainable by phasing out early retirement and improving the mechanism for sick leave compensation. In 2011-12, continuing efforts to reform public administration and the education system would raise efficiency and yield further savings. The planned restructuring of the energy and telecom sectors would draw private investment in, lower costs, and raise the economy's long-term growth rate. Privatization will accelerate as well once investor interest rekindles. Specific plans for reforms beyond 2010 will be fleshed out in the context of program reviews and the 2010 Article IV consultation. The authorities are collaborating closely with the World Bank in the implementation of their ambitious structural agenda.

\section{The program will help mobilize resources for successful implementation of the} poverty reduction agenda. Initially, progress in this area will be guided by the government's poverty reduction strategy named National Development Strategy (NDS), which covers $2008-11$. To promote poverty reduction, the program sets a floor on priority social spending. To benchmark the progress in addressing poverty and refine the strategy, the government will update the NDS by late 2010. The NDS evaluation in the Joint Staff Advisory Note (JSAN) dated February 20, 2008 remains appropriate.

\section{B. Macroeconomic Framework and Risks}

\section{The program's macroeconomic objectives for $\mathbf{2 0 1 0}$ are cautiously optimistic,} reflecting the expected external environment and program policies. Growth is projected to reach $1 \frac{1}{2}$ percent, with evenly balanced risks, on the back of gradual recovery in external and domestic demand and improved business environment. Despite the large output gap, inflation is projected to reach 5 percent by end-2010, pushed mainly by higher international energy prices. The current account deficit would widen modestly to $101 / 4$ percent of GDP from about 9 percent in 2009 reflecting higher prices of imported energy and a recovery in 
investment. Bolstered by sizable official assistance, gross reserves would recover from the fall in 2009.

\section{Over the medium term, the economy should return to its potential, while}

inflation remains low and stable. Growth is expected to reach 5 percent by 2012, led by a rebound in investment and exports, with remittances gradually recovering to their 2008 level. Inflation should remain in mid-single digits, anchored by monetary policy focused on price stability. The current account deficit would eventually stabilize around 8-9 percent of GDP, a level that should be easily financed by FDI and official assistance. It will be helped by the fiscal consolidation and improvements in competitiveness brought by structural reforms and increased exchange rate flexibility. Sizable official assistance and a recovery in private inflows should help reserves grow to comfortable levels.

\begin{tabular}{|c|c|c|c|c|c|c|c|c|}
\hline \multicolumn{9}{|c|}{ Medium-Term Outlook, 2007-14 } \\
\hline & 2007 & 2008 & 2009 & 2010 & 2011 & 2012 & 2013 & 2014 \\
\hline & \multicolumn{8}{|c|}{ Projections } \\
\hline & \multicolumn{8}{|c|}{ (Percent change, unless otherwise indicated) } \\
\hline Real GDP & 3.0 & 7.8 & -9.0 & 1.5 & 3.0 & 5.0 & 5.0 & 5.0 \\
\hline \multirow[t]{2}{*}{ Inflation (end of period, y-o-y) } & 13.1 & 7.4 & 2.5 & 5.0 & 5.0 & 5.0 & 4.0 & 4.0 \\
\hline & \multicolumn{8}{|c|}{ (Percent of GDP, unless otherwise indicated) } \\
\hline Current account balance & -16.5 & -17.3 & -8.9 & -10.2 & -11.2 & -10.2 & -8.9 & -8.4 \\
\hline Private investments & 26.6 & 27.0 & 16.7 & 18.6 & 19.6 & 20.2 & 20.8 & 21.2 \\
\hline Fiscal balance & -0.2 & -1.0 & -9.0 & -7.0 & -5.0 & -3.0 & -1.5 & -0.9 \\
\hline External debt stock & 64.2 & 55.9 & 66.0 & 78.6 & 83.6 & 85.7 & 84.3 & 82.2 \\
\hline $\begin{array}{l}\text { Gross reserves in percent of next year } \\
\text { short-term debt and current account deficit }\end{array}$ & 52.8 & 73.5 & 59.9 & 71.9 & 78.5 & 89.2 & 92.9 & 96.9 \\
\hline
\end{tabular}

Sources: Moldovan authorities; and IMF staff estimates and projections.

18. The medium-term macroeconomic framework may be affected by a possible large credit line from China. The authorities are engaged in preliminary discussions with a Chinese construction company that could undertake public infrastructure projects financed by China's Eximbank up to US\$1 billion (181/2 percent of GDP). The specific projects (in road construction, power supply, etc.) would be executed over the medium term and their consistency with the program - based on updated DSA and macroframework — will be examined as and when they materialize in the context of the program's reviews. Staff's main recommendations to the authorities include phasing disbursements over a number of years and preserving flexibility to adjust the design of the projects as needed to ensure sustainability and achieve the program's objectives. In the event the credit line is utilized as per staff's advice, its macroeconomic implications could be similar to the scenarios in Section IV of the DSA. 


\section{The program allows for sufficient flexibility to deal with economic risks. A}

stronger than envisaged growth recovery would allow faster fiscal consolidation, while the impact of weaker growth on fiscal accounts would be addressed by speeding up the expenditure reform agenda. Lower than expected capital inflows would be cushioned by greater exchange rate flexibility and slower than programmed reserve accumulation; conversely, stronger inflows would imply more ambitious reserve accumulation. Moreover, the authorities have committed to implement additional policy measures if needed to achieve the program targets in case of unfavorable shocks.

\section{The Program for $\mathbf{2 0 1 0}$}

20. In 2010, the program focuses on four main policy challenges:

- $\quad$ Reduce the budget deficit while increasing funds for public investment and social protection spending;

- $\quad$ Relax monetary policy to support growth (as long as inflation remains well below target) and allow substantial exchange rate flexibility;

- $\quad$ Strengthen the NBM's legal toolkit for bank rehabilitation and resolution;

- $\quad$ Arrest the build-up of quasi-fiscal arrears in the energy sector.

\section{The path to fiscal sustainability}

\section{The starting fiscal position} is unsustainable. Spending commitments assumed by the previous government and falling GDP have raised the general government expenditure to a projected $46^{3} / 4$ percent of GDP in 2009, a level typical for higher-income emerging markets (chart), while structural revenue and grants are estimated at 381/4 percent of GDP (table overleaf). In particular, the wage bill in 2009 would surpass 12 percent of GDP — making it one of the highest in Central and Eastern Europe - and the average public sector wage would grow

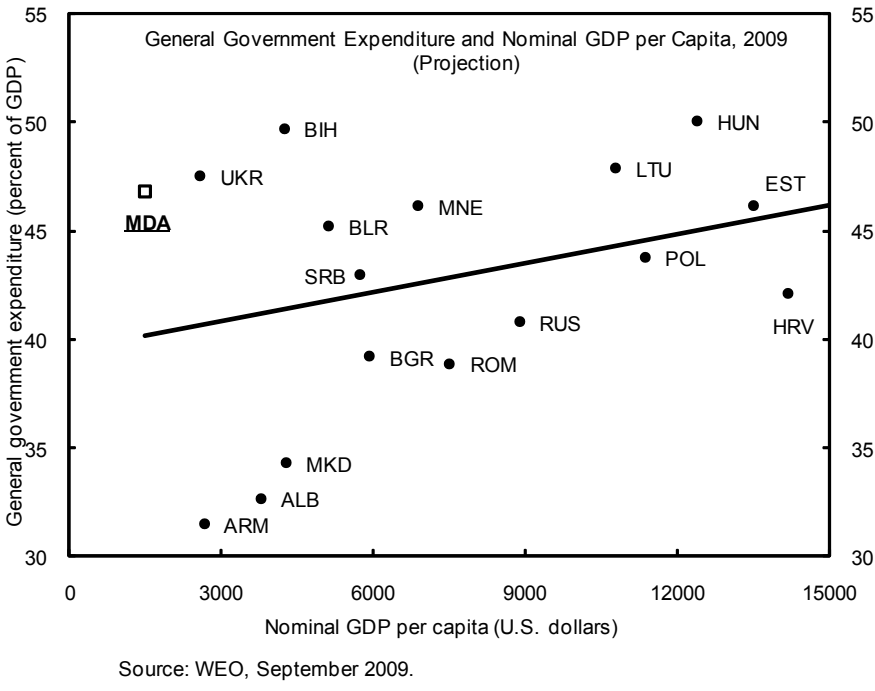

Selected Countries: 2009 Public Sector Wage Bill

\begin{tabular}{lccc}
\hline & $\begin{array}{c}\text { Percent of } \\
\text { GDP }\end{array}$ & $\begin{array}{c}\text { Percent of } \\
\text { government } \\
\text { expenditure }\end{array}$ & $\begin{array}{c}\text { 2009 average real } \\
\text { wage increase, } \\
\text { percent }\end{array}$ \\
\hline Lithuania & 14.7 & 30.8 & -11.7 \\
Moldova & $\mathbf{1 2 . 1}$ & $\mathbf{2 5 . 8}$ & $\mathbf{1 9 . 2}$ \\
Moldova, 2008 & $\mathbf{9 . 1}$ & $\mathbf{2 1 . 9}$ & $\ldots$ \\
Ukraine & 11.6 & 24.5 & -10.9 \\
Romania & 9.2 & 24.2 & -5.2 \\
Estonia & 8.4 & 16.9 & $\ldots$ \\
Belarus & 6.7 & 19.4 & 0.9 \\
Bulgaria & 6.4 & 16.4 & 1.4 \\
Armenia & 2.3 & 8.7 & 8.0 \\
\hline Soures: & & &
\end{tabular}


by almost 20 percent in real terms, in sharp contrast with other countries in the region (table). A structural deficit of $8 \frac{1}{2}$ percent of GDP cannot be financed from sources usually available to Moldova.

Headline and Structural Fiscal Balances, 2008-12

(General government; percent of GDP)

\begin{tabular}{|c|c|c|c|c|c|c|}
\hline & \multirow[t]{2}{*}{2008} & 2009 & \multicolumn{2}{|c|}{2010} & \multirow{2}{*}{$\begin{array}{l}2011 \\
\text { Prog. }\end{array}$} & \multirow{2}{*}{$\begin{array}{l}2012 \\
\text { Prog. }\end{array}$} \\
\hline & & Proj. & $\begin{array}{l}\text { Proj. w/o } \\
\text { measures }\end{array}$ & Prog. & & \\
\hline Headline revenue and grants & 40.6 & 37.8 & 36.7 & 39.0 & 39.2 & 39.3 \\
\hline Structural revenue & 35.6 & 36.2 & 36.4 & 37.0 & 37.5 & 37.8 \\
\hline Grants & 1.7 & 2.1 & 1.4 & 3.2 & 2.5 & 1.8 \\
\hline Automatic stabilizers & 3.3 & -0.5 & -1.2 & -1.2 & -0.7 & -0.3 \\
\hline Expenditure and net lending & 41.6 & 46.8 & 50.7 & 46.0 & 44.2 & 42.3 \\
\hline Current & 34.5 & 41.6 & 44.7 & 40.0 & 37.3 & 35.4 \\
\hline Capital & 7.0 & 5.3 & 6.1 & 6.1 & 7.0 & 7.0 \\
\hline Headline fiscal balance & -1.0 & -9.0 & -14.0 & -7.0 & -5.0 & -3.0 \\
\hline Structural fiscal balance (incl. grants) & -4.3 & -8.5 & -12.8 & -5.8 & -4.3 & -2.7 \\
\hline
\end{tabular}

Source: IMF staff estimates.

22. The program seeks to balance the needed sizable fiscal adjustment with large public investment and social spending needs. On unchanged policies the commitmentbased budget deficit would have reached 14 percent of GDP in 2010. To bring the deficit down, the authorities have rescheduled large unaffordable wage increases and rationalized spending on goods and services and subsidies (MEFP $q 13$ ). Increases in consumption-based taxes will help the adjustment as well. At the same time, the budget envisages an increase in public investment by 26 percent to support growth, and a rise in social assistance spending by 36 percent to help vulnerable households weather the crisis and transition to the new targeted social assistance scheme without a break in benefits

Planned Fiscal Measures in 2010 1/

\begin{tabular}{lc}
\hline \multicolumn{1}{c}{ Measure } & $\begin{array}{c}\text { Effect } \\
\text { (Percent of GDP) }\end{array}$ \\
\hline Expenditure & 4.7 \\
Reduce wage bill & 3.6 \\
Rationalize spending on goods and services & 0.7 \\
Reduce subsidies & 0.4 \\
Revenue & 0.8 \\
Excise tax measures & 0.9 \\
VAT measures & 0.2 \\
Effect of the reduced public sector wage bill & -0.4 \\
\cline { 2 - 2 } Total & 5.4 \\
\hline
\end{tabular}

Source: IMF staff estimates.

1/ Relative to unchanged policies

(MEFP $\mid 25)$. Together with increased external grants, these measures result in a deficit target of 7 percent of GDP in 2010, a structural adjustment of about $23 / 4$ percentage points of GDP 
from 2009. The authorities and staff agreed that should revenue underperform, further measures may need to be implemented to keep the targeted adjustment.

23. Structural reforms with medium-term payoff will support the fiscal effort. To improve the financial sustainability of the social insurance system, early retirement privileges of civil servants, judges, and prosecutors will begin to be phased out in 2010, and sick leave compensation would be revamped (MEFP $\mid 23$ ). Motivated by a steadily declining student population, the authorities will begin closing redundant education institutions and freezing enrollment in pedagogical colleges.

24. To ensure adequate budget financing in 2010, the authorities request that the equivalent of SDR 95 million (US\$150 million) of the access under the arrangements be used for direct budget support. Moldova lacks access to international capital markets and the domestic market for government securities has limited capacity. The alternatives to Fund financing are sharp procyclical expenditure cuts, accumulation of arrears, or central bank budget financing (currently prohibited by law), which would compromise NBM's credibility with potentially destabilizing effects on the exchange rate and inflation. According to a Memorandum of Understanding (MoU) between the Ministry of Finance and the NBM, the domestic-currency counterpart of this amount, split between the first three tranches (MEFP Table 1), will be deposited in the Ministry of Finance's account with the NBM, while the foreign-currency counterpart will augment the NBM's reserves, alleviating the balance of payments need. The MoU also specifies the procedures for repayments of the funds for budget support. The programmed fiscal adjustment for 2011 and beyond will ensure that the budget will not become permanently dependent on Fund resources.

\section{Easier monetary policy to support growth while maintaining low inflation}

25. As inflation remains very low, the NBM aims to ease policy in the short run, consistent with the 2010 inflation objective of 5 percent. Given the large premium incorporated in bank lending rates and ample bank liquidity, further base rate and reserve requirement cuts are unlikely to make a notable difference. To discourage bank hoarding of liquidity and promote credit expansion, the NBM has restarted open market operations with a view to assure banks that liquidity support—against good collateral—will be available if needed. Moreover, the NBM plans substantial real reserve money growth in 2010 ( $81 / 2$ percent on average, after a contraction by 7 percent in 2009), which will pressure bank interest rates down. Should the 2010 inflation objective be threatened, however, monetary policy will adjust promptly.

26. The NBM's intervention in the foreign exchange market will be limited to counteracting erratic fluctuations without resisting trends. Moldova's shallow foreign exchange market justifies occasional intervention to resist sharp exchange rate movements 
triggered by random events. ${ }^{1}$ However, resistance of sustained trends should be avoided as it slows down the adjustment of the economy to shocks and wastes precious reserves. The NBM has committed to allow substantial exchange rate flexibility, while using intervention as an exceptional tool in times of heightened volatility in line with its inflation objective. Staff analysis indicates that the banks' direct and indirect credit risk from exchange rate movements is manageable, as currency mismatches are limited and borrowing in foreign currencies is subject to strict rules.

27. After the recent correction, the real exchange rate seems broadly in line with fundamentals. Versions of the macroeconomic balance approach span a range from moderate overvaluation of 7 percent to slight undervaluation of 2 percent, suggesting that the projected medium-term current account deficit is in line with the norm. The external sustainability approach suggests at most moderate overvaluation of 10 percent. These estimates need to be interpreted cautiously, given large uncertainties about structural changes in the economy that can impact productivity and patterns of capital inflows.

28. Taking advantage of the return of confidence in the leu, the NBM is replenishing its foreign exchange reserves. Specifically, gross international reserves increased to almost US\$1500 million at end-2009 to create a comfortable buffer against external shocks. This corresponds to a cover of 4.4 months of 2010 imports and 87 percent of short-term debt (Table 2). Staff calculations of optimal level of reserves yield a higher estimate for 2010 (US\$1550 million), which will be reached early in the year. ${ }^{2}$ The ensuing injection of liquidity is in line with targeted money growth.

\section{Strengthening of bank resolution framework and financial sector monitoring}

29. Prompted by the Investprivatbank failure, the NBM has been working on strengthening its ability to intervene early in problem banks and improve the effectiveness of the Deposit Guarantee Fund (DGF; MEFP 119). Legal amendments will introduce the instrument of "official administration" through which the NBM can, prior to revoking their license, establish control over troubled banks to rehabilitate or resolve them. Another set of amendments will ensure prompt payment to insured depositors from the DGF in case of a bank failure, as well as improve the DGF's governance structure.

30. The NBM is closely monitoring banks' financial soundness and stands ready to take preemptive action as needed (MEFP q20). It will continue to perform monthly stress testing of each bank's balance sheet and periodically review the banks' internal control and risk management systems. The results of the stress tests and the ongoing independent

\footnotetext{
1 "Intervention" is meant in the narrow sense as an action aimed to influence the exchange rate.

${ }^{2}$ See Jeanne, O. and R. Ranciere (2006), “The Optimal Level of International Reserves for Emerging Market Countries: Formulas and Applications ”, IMF Working Paper No. 06/229.
} 
diagnostics study of banks' portfolios will be used for early detection of the need for capital injections so that banks' capital-asset ratios remain safely above the statutory ratio of 12 percent.

\section{Stemming the tide of quasi-fiscal arrears in the heating sector}

\section{In collaboration with the World Bank, the authorities have depoliticized the} setting of heating tariffs and raised the tariff in Chisinau. Parliament transferred the responsibility for tariff setting from the municipal authorities to the energy regulator ANRE. In January, ANRE adjusted the heating tariff in Chisinau to a level sufficient to cover heating costs, ensuring current payments to suppliers (MEFP $\mid 24$ ). The municipality supports lowincome households by targeted assistance for heating. With the assistance of the World Bank, the authorities are considering a plan to clear the stock of accumulated arrears.

\section{Program Modalities}

\section{A. Access and Phasing}

32. Moldova faces protracted balance of payments needs. To prevent sharp growthreducing contraction in imports and secure a comfortable level of reserves, staff estimate that external financing of US\$1.25 billion will be required over 2010-12, including US\$633 million for reserve accumulation (Table 6). The programmed level of reserves is adequate to cushion shortfalls in private inflows should such shortfalls emerge.

33. The program is fully financed. From the Fund, staff propose total access of 300 percent of quota (SDR 369.6 million, US\$588 million, about half of needed funds), split evenly between 36-month ECF and EFF. ${ }^{3}$ This choice of facilities reflects Moldova's protracted balance of payments need and the significant structural transformation that is needed to stabilize the economy and ensure sustainable growth. Staff propose that SDR 60 million be disbursed upfront, with the remainder coming in semi-annual tranches upon conclusion of program reviews. The access would be somewhat frontloaded, with almost half of the funds made available in 2010. Such a schedule would address the most critical balance of payments needs as the economy remains exposed to the global economic crisis. To better balance the capacity to repay, staff propose to frontload the concessional resources, with increasing share of access to GRA funds later in the program period when economic conditions are expected to improve (Table 7). Other partners - the European

\footnotetext{
${ }^{3}$ Moldova is eligible to use the Fund's concessional facilities and is presumed to use a blend of concessional and GRA resources, given its relatively high per capita income level. Consistent with the blending policy, concessional and GRA resources are used in equal proportions and the concessional portion does not exceed an annual average of 50 percent of quota. At end-2009, Moldova's outstanding concessional credit from the Fund was 79.7 percent of quota.
} 
Commission, the World Bank, the EIB, the EBRD, and the CEDB-would provide the other half of the financial package (Table 6).

\section{B. Capacity to Repay the Fund and Risks to the Program}

34. Moldova's capacity to repay is good. Fund exposure is projected to peak at 12.2 percent of GDP in 2012. Total debt service to the Fund would reach 1.1 percent of total exports in 2014, or 0.6 percent of GDP, and peak at 2.7 percent of exports in 2017, or $1 \frac{1}{2}$ percent of GDP. Under current projections, the stock of external public and publicly guaranteed debt would peak at $34 \frac{1}{2}$ percent of GDP by 2012. The blend between concessional and GRA resources and their proposed phasing will ensure that the increase in repurchase obligations be gradual and temporary.

35. Nevertheless, some repayment risks exist. Owing to unusually large private debt for a LIC, total external debt is projected to reach 86 percent in 2012, and debt service will remain high at 19 percent of exports of goods and services by 2014 . While private debt rollover risk appears limited, private debt service relies for timely payments on the same pool of resources as its public counterpart, thus posing some risks to Moldova's ability to service its obligations to the Fund. Moreover, significant frontloading of the possible loans for infrastructure projects from China could raise the debt burden in the medium term. Finally, Moldova has had instances of debt distress when external financing turned scarce, namely a Eurobond rescheduling in 2002 and a partial Paris Club debt rescheduling in 2006.

36. Moreover, program implementation is subject to political risks. Inability to elect a President may provoke elections in the second half of 2010 with attendant risks for program policies and objectives. Such a development may reduce external financial support, pressuring the balance of payments again.

37. The program includes policies to mitigate the risks to program implementation and to Moldova's capacity to repay the Fund. Key fiscal and reserve accumulation measures were prior actions for the Board consideration of the request for the use of Fund facilities. Programmed reserve accumulation would help secure the necessary resources for timely repayments of external liabilities. Finally, large foreign-financed project loans would trigger additional fiscal adjustment, thus alleviating repayment risks posed by the possible significant bilateral nonconcessional borrowing in the medium term.

\section{Program Monitoring and Conditionality}

38. The program will be monitored through semi-annual reviews, prior actions, quantitative performance criteria and indicative targets, and structural benchmarks (MEFP Tables 1-3). The first review will be based on end-March 2010 targets. Quantitative performance criteria and indicative targets reflect the program's main policy objectives 
(MEFP q27 and MEFP Table 2). ${ }^{4}$ Structural benchmarks for the first review include actions to strengthen the bank resolution framework and extend the new targeted social assistance system.

39. Safeguards. An updated safeguards assessment will be completed by the first review under the arrangements. The safeguards mission will also review the MoU between the Ministry of Finance and the NBM on the use of Fund disbursements for budget support. The authorities have already provided the documentation necessary to complete the update and have authorized their external auditors to hold discussions with Fund staff.

\section{Staff Appraisal}

40. Moldova's macroeconomic developments and policies deteriorated sharply in 2009. Exports, remittances, and FDI fell dramatically as a result of the downturn in trading partners. Exacerbating the effects of the crisis, fiscal policy fell prey to the electoral cycle, while monetary policy remained too tight defending the exchange rate, despite some relaxation since May.

41. Restoring fiscal and external sustainability will require steady adherence to the authorities' adjustment program and the provision of adequate external financing. Strong policies will ensure that the deterioration in the structural budget balance is reversed; inflation is kept under control while international reserves are brought up to adequate levels; and the banking system remains stable. External financing support from Moldova's development partners would cushion the economy from the worst effects of the global crisis and allow a return to macroeconomic stability.

42. The fiscal strategy appropriately balances needed adjustment with large public investment and social needs. The programmed fiscal adjustment equivalent to 6 percent of GDP over three years would restore a manageable structural fiscal balance while providing for a sizable increase in funds for public investment and social protection. The possible credit line from China could help upgrade public infrastructure and the economy's export potential, subject to being consistent with debt sustainability and the program's stability objectives.

\section{Prudent monetary policy management and the introduction of a clear and well- communicated policy framework will contribute a great deal to the stabilization and}

\footnotetext{
${ }^{4}$ The program includes as a performance criterion a ceiling of US $\$ 125$ million on contracting or guaranteeing of nonconcessional external debt by the general government. This ceiling is consistent with keeping government debt in safe territory, as shown in section IV of the DSA. Conditionality on debt accumulation will be reviewed at the time of the first review, in light of the new policy on debt limits. Moldova was assessed to be a higher capacity/lower debt vulnerability country, and could therefore be eligible to the more flexible conditionality options under the new policy. Such options were not considered for the program request, as discussions with the authorities took place before the new policy was implemented.
} 
growth of the economy. As inflation remains well below the NBM's target, monetary policy can ease further and support output recovery. The new NBM policy framework should help reduce the risk premia embedded in commercial bank interest rates and thus raise monetary policy effectiveness while fostering financial development. The framework envisages substantial exchange rate flexibility and only occasional interventions to calm disorderly markets.

44. Financial sector measures will improve the NBM's ability to resolve troubled banks and raise confidence in banks' financial soundness. The forthcoming legal amendments will give the NBM additional tools for early action on troubled banks and strengthen the reliability of the Deposit Guarantee Fund. The results of the ongoing diagnostic procedures by independent auditors will clarify the condition of commercial banks' portfolios, thus removing a key source of uncertainty regarding bank soundness. Should the diagnostics reveal the need for preemptive measures, the NBM has committed to act swiftly as so far.

45. The heating sector losses and arrears are beginning to be addressed. The transfer of responsibility for heating tariff setting to the energy regulator and the implemented tariff adjustment are welcome steps lessening the risks to the budget and to the supply of heat.

46. The program is subject to certain risks. These include slower than projected recovery in trading partners and the possibility of waning political support should elections be held in late 2010. While the program design and conditionality safeguard against these risks, continuous policy dialogue with the Fund would remain essential for the success of the program, along with the commitment to adjust policies as needed to achieve program objectives.

47. In view of Moldova's balance of payments needs and the strong program proposed by the authorities, staff supports the authorities' request for combined 3-year ECF/EFF in the amount of SDR 369.6 million. Steadfast implementation of the program will help Moldova overcome current difficulties and restore macroeconomic stability and growth. 
Figure 1. Moldova: A Difficult Macroeconomic Situation
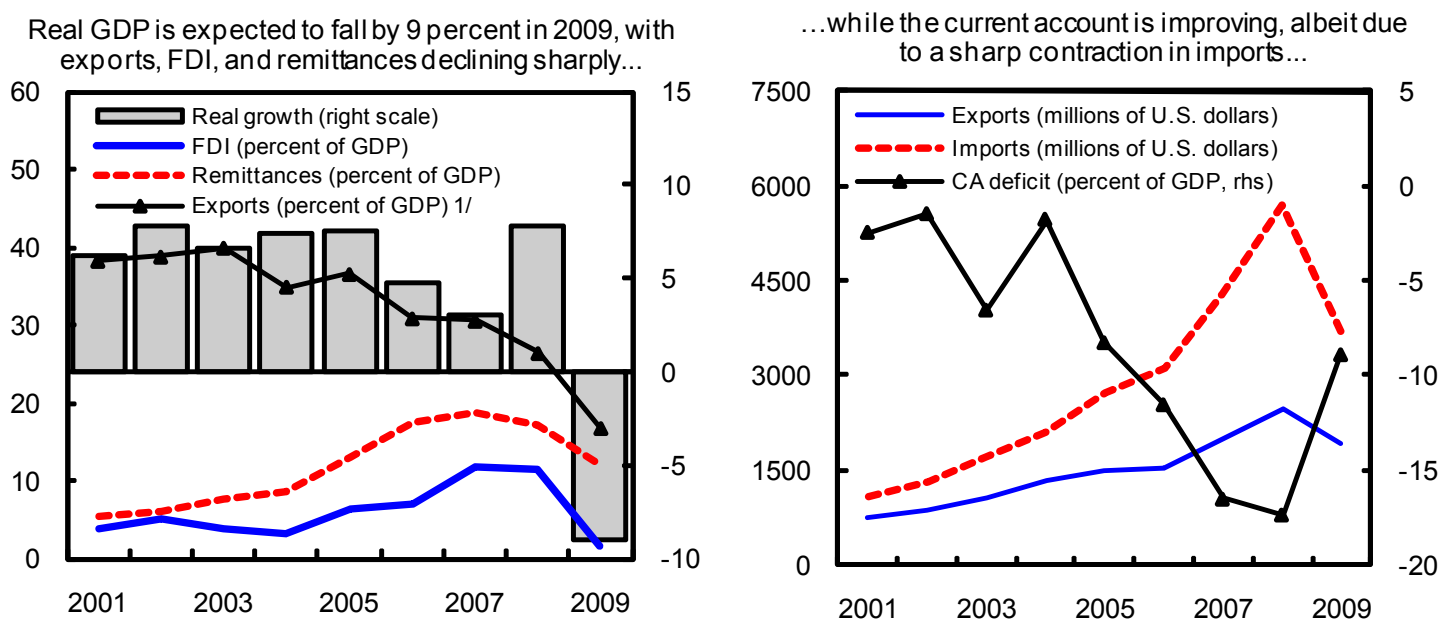

...with deflation pressures, reflecting both the economic downturn and declining commodity prices.

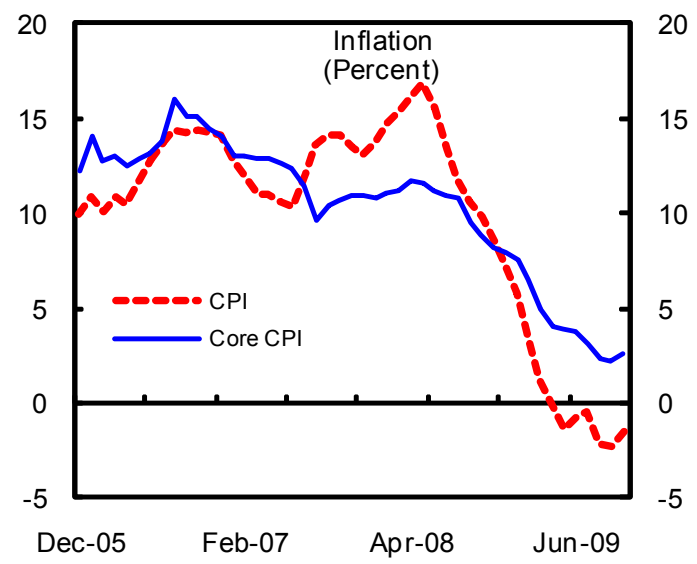

The NBM has begun to recoup lost reserves and the REER has stabilized at its new level ...

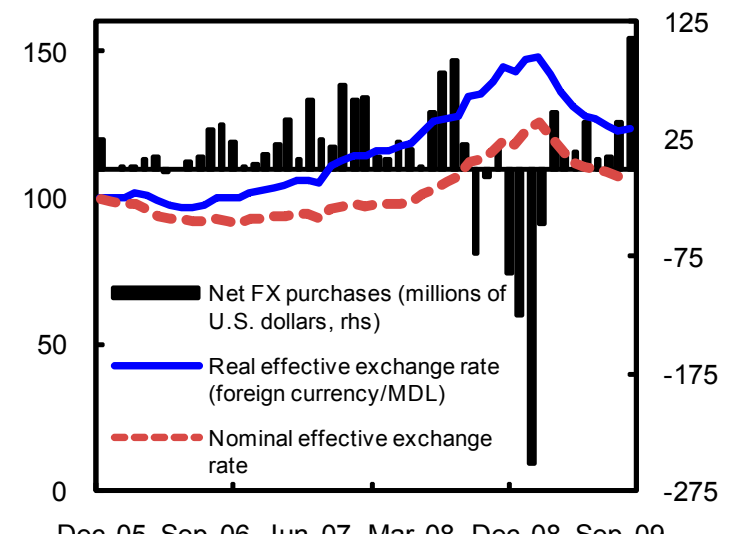

Dec-05 Sep-06 Jun-07 Mar-08 Dec-08 Sep-09

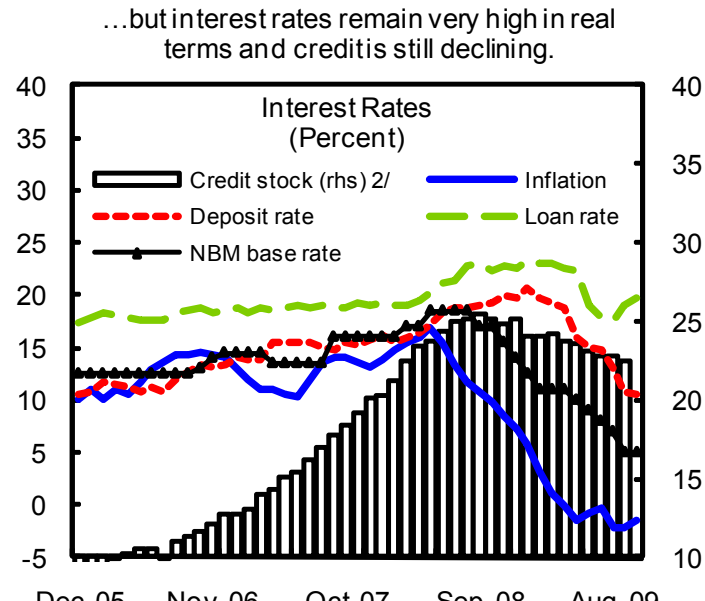

The budget has run into huge deficit, with revenue declining and expenditure increasing.

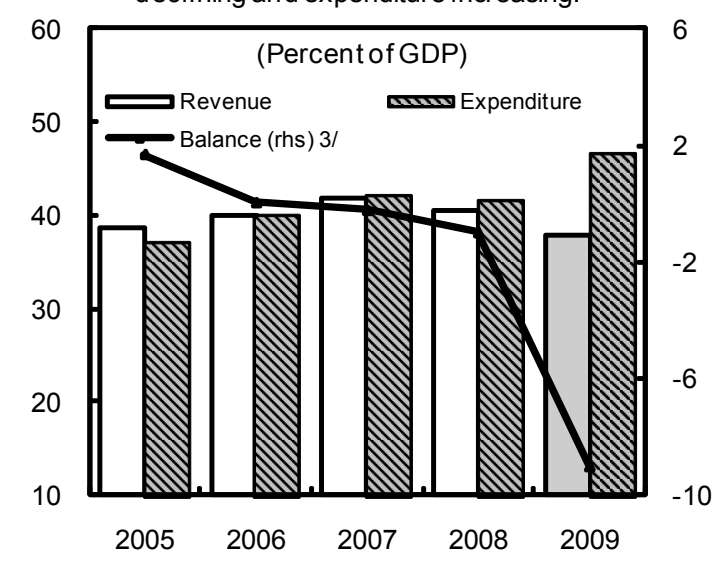

Sources: IMF, World Economic Outlook, October 2009; National Bureau of Statistics; and IMF staff calculations. $1 /$ Data as of September 2009.

2/In billions of Moldovan lei at a constant exchange rate of MDL10.4/US\$1 as of end-2008.

$3 /$ Refers to overall balance. 
Table 1. Moldova: Selected Indicators, 2007-14 1/

\begin{tabular}{|c|c|c|c|c|c|c|c|c|}
\hline & 2007 & 2008 & 2009 & 2010 & 2011 & 2012 & 2013 & 2014 \\
\hline & & & \multicolumn{6}{|c|}{ Projection } \\
\hline I. Real sector indicators & \multicolumn{8}{|c|}{ (Percent change, unless otherwise indicated) } \\
\hline \multicolumn{9}{|l|}{ Gross domestic product } \\
\hline Real growth rate & 3.0 & 7.8 & -9.0 & 1.5 & 3.0 & 5.0 & 5.0 & 5.0 \\
\hline Demand & 7.8 & 5.7 & -18.9 & 2.3 & 3.3 & 4.2 & 4.7 & 4.8 \\
\hline Consumption & 3.8 & 5.7 & -7.0 & -1.7 & 0.4 & 3.5 & 3.6 & 4.2 \\
\hline Private & 1.8 & 5.8 & -10.6 & -1.1 & 1.4 & 4.8 & 3.7 & 4.3 \\
\hline Public & 13.5 & 5.0 & 9.5 & -3.5 & -3.1 & -1.1 & 3.1 & 3.8 \\
\hline Gross fixed capital formation & 25.5 & 2.2 & -38.4 & 6.1 & 10.2 & 7.2 & 9.2 & 7.4 \\
\hline Private & 33.9 & 4.1 & -41.0 & 5.0 & 8.0 & 8.0 & 8.0 & 8.0 \\
\hline Public & 2.9 & -4.6 & -28.4 & 9.5 & 16.8 & 4.8 & 12.8 & 5.7 \\
\hline Nominal GDP (billions of Moldovan lei) & 53.4 & 62.9 & 59.5 & 64.3 & 69.4 & 76.2 & 83.5 & 91.2 \\
\hline Nominal GDP (billions of U.S. dollars) & 4.4 & 6.1 & 5.4 & 5.1 & 5.3 & 5.7 & 6.1 & 6.6 \\
\hline Consumer price index (average) & 12.4 & 12.7 & 0.3 & 6.2 & 5.0 & 5.0 & 4.5 & 4.0 \\
\hline Consumer price index (end of period) & 13.1 & 7.4 & 2.5 & 5.0 & 5.0 & 5.0 & 4.0 & 4.0 \\
\hline GDP deflator & 15.9 & 9.2 & 3.9 & 6.5 & 4.7 & 4.7 & 4.3 & 4.0 \\
\hline Average monthly wage (Moldovan lei) & 2,063 & 2,529 & 2,750 & $\ldots$ & $\ldots$ & $\ldots$ & $\ldots$ & $\ldots$ \\
\hline Average monthly wage (U.S. dollars) & 170 & 243 & 248 & $\ldots$ & $\ldots$ & $\ldots$ & $\ldots$ & $\ldots$ \\
\hline Saving-investment balance & \multicolumn{8}{|c|}{ (Percent of GDP) } \\
\hline Foreign saving & 16.5 & 17.3 & 8.9 & 10.2 & 11.2 & 10.2 & 8.9 & 8.4 \\
\hline National saving & 17.6 & 16.7 & 13.1 & 14.5 & 15.4 & 17.1 & 19.3 & 20.3 \\
\hline Private & 10.3 & 10.6 & 16.8 & 15.4 & 13.4 & 13.1 & 13.3 & 13.7 \\
\hline Public & 7.3 & 6.0 & -3.7 & -0.8 & 2.0 & 4.0 & 6.0 & 6.6 \\
\hline Gross investment & 34.1 & 34.0 & 22.0 & 24.7 & 26.6 & 27.2 & 28.3 & 28.7 \\
\hline Private & 26.6 & 27.0 & 16.7 & 18.6 & 19.6 & 20.2 & 20.8 & 21.2 \\
\hline Public & 7.5 & 7.0 & 5.3 & 6.1 & 7.0 & 7.0 & 7.5 & 7.5 \\
\hline \multicolumn{9}{|l|}{ II. Fiscal indicators (general government) } \\
\hline Primary balance (cash) & 1.0 & 0.2 & -7.6 & -5.7 & -3.7 & -1.9 & -0.5 & 0.0 \\
\hline Overall balance (cash) & -0.2 & -1.0 & -9.0 & -7.0 & -5.0 & -3.0 & -1.5 & -0.9 \\
\hline Stock of general government debt & 26.8 & 21.3 & 30.9 & 36.9 & 39.6 & 41.0 & 38.6 & 36.0 \\
\hline III. Financial indicators & \multicolumn{8}{|c|}{ (End of period percent change, unless otherwise indicated) } \\
\hline Broad money (M3) & 39.8 & 15.9 & -0.2 & 9.3 & $\ldots$ & $\ldots$ & $\ldots$ & $\ldots$ \\
\hline Velocity (GDP/end-period M3; ratio) & 2.0 & 2.0 & 1.9 & 1.9 & $\ldots$ & $\ldots$ & $\ldots$ & $\ldots$ \\
\hline Reserve money & 46.4 & 22.0 & -6.3 & 7.4 & $\ldots$ & $\ldots$ & $\ldots$ & $\ldots$ \\
\hline Credit to the economy & 51.7 & 20.3 & -7.1 & 10.7 & $\ldots$ & $\ldots$ & $\ldots$ & $\ldots$ \\
\hline IV. External sector indicators & \multicolumn{8}{|c|}{ (Millions of U.S. dollars, unless otherwise indicated) } \\
\hline Current account balance & -726 & $-1,049$ & -478 & -518 & -597 & -579 & -547 & -554 \\
\hline Current account balance (percent of GDP) & -16.5 & -17.3 & -8.9 & -10.2 & -11.2 & -10.2 & -8.9 & -8.4 \\
\hline Remittances and compensation of employees (net) & 1,419 & 1,796 & 1,135 & 1,237 & 1,363 & 1,530 & 1,695 & 1,871 \\
\hline Gross official reserves & 1,334 & 1,672 & 1,456 & 1,695 & 1,875 & 2,089 & 2,245 & 2,444 \\
\hline Gross official reserves (months of imports) & 2.8 & 5.5 & 4.4 & 4.5 & 4.5 & 4.6 & 4.6 & 4.6 \\
\hline Real effective exchange rate, end-year, change (percent) & 16.0 & 23.3 & -19.7 & $\ldots$ & $\ldots$ & $\ldots$ & $\ldots$ & \\
\hline External debt (percent of GDP) $2 /$ & 64.2 & 55.9 & 66.0 & 78.6 & 83.6 & 85.7 & 84.3 & 82.2 \\
\hline Debt service (percent of exports of goods and services) & 13.7 & 16.6 & 19.2 & 20.8 & 16.8 & 18.3 & 16.8 & 19.2 \\
\hline
\end{tabular}

Sources: Moldovan authorities; and IMF staff estimates and projections.

1/ Data exclude Transnistria.

2/ Includes private and public debt. 
Table 2. Moldova: Balance of Payments, 2007-14

(Millions of U.S. dollars, unless otherwise indicated)

\begin{tabular}{|c|c|c|c|c|c|c|c|c|}
\hline & 2007 & 2008 & 2009 & 2010 & 2011 & 2012 & 2013 & 2014 \\
\hline & & & \multicolumn{6}{|c|}{ Projection } \\
\hline Current account balance & -726 & $-1,049$ & -478 & -518 & -597 & -579 & -547 & -554 \\
\hline Merchandise trade balance & $-2,303$ & $-3,220$ & $-1,775$ & $-1,909$ & $-2,059$ & $-2,174$ & $-2,323$ & $-2,490$ \\
\hline Exports & 1,373 & 1,646 & 1,325 & 1,448 & 1,762 & 2,000 & 2,228 & 2,461 \\
\hline Of which: wine and alcohol & 136 & 196 & 174 & 181 & 184 & 195 & 209 & 225 \\
\hline Imports & $-3,676$ & $-4,866$ & $-3,100$ & $-3,357$ & $-3,821$ & $-4,174$ & $-4,551$ & $-4,950$ \\
\hline Services balance & -6 & 12 & 9 & 9 & 21 & 31 & 43 & 55 \\
\hline Exports of services & 625 & 837 & 582 & 632 & 745 & 832 & 921 & 1,016 \\
\hline Imports of services & -631 & -825 & -574 & -623 & -724 & -800 & -879 & -961 \\
\hline Income balance & 416 & 598 & 319 & 265 & 312 & 349 & 404 & 430 \\
\hline Compensation of employees & 593 & 763 & 489 & 536 & 604 & 675 & 744 & 819 \\
\hline Income on direct and portfolio investment & -170 & -141 & -118 & -178 & -187 & -200 & -214 & -230 \\
\hline Income on other investment & -6 & -24 & -52 & -93 & -106 & -126 & -126 & -159 \\
\hline Current transfer balance & 1,167 & 1,561 & 969 & 1,116 & 1,129 & 1,215 & 1,330 & 1,451 \\
\hline Remittances & 826 & 1,033 & 646 & 700 & 759 & 855 & 952 & 1,052 \\
\hline Budget transfers & 73 & 120 & 82 & 161 & 131 & 103 & 103 & 103 \\
\hline Other transfers & 267 & 408 & 241 & 255 & 240 & 257 & 275 & 296 \\
\hline Capital and financial account balance & 995 & 1,253 & 276 & 651 & 628 & 656 & 726 & 786 \\
\hline Capital account balance & -8 & -15 & -11 & -11 & -11 & -12 & -13 & -14 \\
\hline Financial account balance & 1,003 & 1,268 & 287 & 661 & 640 & 668 & 739 & 800 \\
\hline Foreign direct investment balance & 522 & 691 & 91 & 197 & 259 & 295 & 363 & 423 \\
\hline Portfolio investment and derivatives & -5 & 7 & -6 & -6 & -6 & -5 & -5 & -6 \\
\hline Other investment balance & 485 & 569 & 202 & 470 & 387 & 378 & 381 & 382 \\
\hline Loans & 272 & 356 & 165 & 370 & 307 & 298 & 296 & 290 \\
\hline General government, net $1 /$ & -15 & -21 & 145 & 245 & 121 & 108 & 88 & 65 \\
\hline Private sector, net & 287 & 378 & 20 & 126 & 186 & 190 & 208 & 225 \\
\hline Other capital flows & 213 & 212 & 37 & 100 & 80 & 80 & 86 & 92 \\
\hline SDR allocation & & $\ldots$ & 175 & $\ldots$ & $\ldots$ & $\ldots$ & $\ldots$ & $\ldots$ \\
\hline Errors and omissions & 114 & 77 & -67 & 0 & 0 & 0 & 0 & 0 \\
\hline Overall balance & 383 & 280 & -269 & 132 & 32 & 77 & 179 & 232 \\
\hline Financing & -383 & -280 & 269 & -131 & -31 & -76 & -178 & -231 \\
\hline Gross international reserves (increase: -) & -529 & -452 & 217 & -239 & -180 & -214 & -156 & -198 \\
\hline Use of Fund credit, net & 11 & 12 & -15 & 108 & 149 & 138 & -22 & -32 \\
\hline Purchases 2/ & 33 & 38 & 0 & 117 & 155 & 155 & 0 & 0 \\
\hline Repurchases & -22 & -25 & -15 & -9 & -6 & -16 & -22 & -32 \\
\hline Exceptional financing & 135 & 160 & 69 & 0 & 0 & 0 & 0 & 0 \\
\hline Memorandum items: & \multicolumn{8}{|c|}{ (Percents of GDP, unless otherwise indicated) } \\
\hline Financing gap before IMF arrangement and donors' assistance & $\ldots$ & $\ldots$ & $\ldots$ & 521 & 383 & 344 & $\ldots$ & $\ldots$ \\
\hline Gross official reserves (millions of U.S. dollars) $3 /$ & 1,334 & 1,672 & 1,456 & 1,695 & 1,875 & 2,089 & 2,245 & 2,444 \\
\hline Months of imports of good and services & 2.8 & 5.5 & 4.4 & 4.5 & 4.5 & 4.6 & 4.6 & 4.6 \\
\hline Percent of short-term debt at remaining maturity & 89.1 & 101.3 & 87.2 & 103.4 & 110.2 & 122.4 & 124.9 & 132.9 \\
\hline Current account balance & -16.5 & -17.3 & -8.9 & -10.2 & -11.2 & -10.2 & -8.9 & -8.4 \\
\hline Goods and services trade balance & -52.5 & -53.0 & -33.0 & -37.3 & -38.2 & -37.6 & -37.3 & -37.0 \\
\hline Exports of goods and services & 45.4 & 41.0 & 35.6 & 40.8 & 47.0 & 49.6 & 51.5 & 52.8 \\
\hline Imports of goods and services & -97.9 & -94.0 & -68.5 & -78.1 & -85.3 & -87.2 & -88.7 & -89.8 \\
\hline Remittances & 32.2 & 29.7 & 21.2 & 24.3 & 25.6 & 26.8 & 27.7 & 28.4 \\
\hline Foreign direct investment balance & 11.9 & 11.4 & 1.7 & 3.9 & 4.9 & 5.2 & 5.9 & 6.4 \\
\hline Total debt service (percent of exports of goods and services) & 13.7 & 16.6 & 19.2 & 20.8 & 16.8 & 18.3 & 16.8 & 19.2 \\
\hline
\end{tabular}

Sources: Moldovan authorities; and IMF staff estimates and projections.

1/ For 2010, projections include IMF disbursement to the Ministry of Finance amounting to US\$150 million.

2/ For 2010, total IMF disbursement is projected to amount to US\$267 million, of which US\$150 million will be disbursed directly to the Ministry of Finance, and included in the financial account as indicated in footnote 1.

$3 /$ Includes revaluation changes, which are not captured by changes of gross official reserves in the BOP. 
Table 3. Moldova: General Government Budget, 2007-14

(Millions of Moldovan lei, unless otherwise indicated)

\begin{tabular}{|c|c|c|c|c|c|c|c|c|c|}
\hline \multirow{3}{*}{$\begin{array}{l}\text { Revenues and grants } \\
\text { Re }\end{array}$} & \multirow{3}{*}{$\begin{array}{r}2007 \\
22,292\end{array}$} & \multirow{3}{*}{ 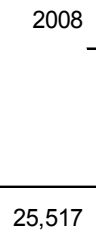 } & \multirow{3}{*}{$\begin{array}{r}2009 \\
\text { Proj. } \\
22,469\end{array}$} & \multicolumn{2}{|c|}{2010} & \multirow{2}{*}{$\begin{array}{c}2011 \\
\text { Prog. }\end{array}$} & \multirow{2}{*}{$\begin{array}{l}2012 \\
\text { Prog. }\end{array}$} & \multirow{2}{*}{$\begin{array}{l}2013 \\
\text { Proj. }\end{array}$} & \multirow{2}{*}{$\begin{array}{l}2014 \\
\text { Proj. }\end{array}$} \\
\hline & & & & $\begin{array}{c}\text { Proj. } \\
\text { without } \\
\text { measures }\end{array}$ & Prog. & & & & \\
\hline & & & & 23,595 & 25,105 & 27,186 & 29,939 & 34,043 & 37,373 \\
\hline Revenues & 19,798 & 22,604 & 19,576 & 21,067 & 21,569 & 23,722 & 26,629 & 30,520 & 33,635 \\
\hline Tax revenues & 18,171 & 21,030 & 18,597 & 19,946 & 20,448 & 22,581 & 25,375 & 29,146 & 32,135 \\
\hline Profit tax & 1,388 & 718 & 356 & 216 & 216 & 316 & 347 & 1,217 & 1,339 \\
\hline Personal income tax & 1,329 & 1,480 & 1,393 & 1,524 & 1,493 & 1,631 & 1,793 & 1,964 & 2,358 \\
\hline VAT & 7,587 & 9,097 & 7,393 & 8,053 & 8,188 & 9,132 & 10,432 & 11,738 & 12,904 \\
\hline Excises & 1,392 & 1,574 & 1,388 & 1,388 & 1,995 & 2,018 & 2,280 & 2,508 & 2,652 \\
\hline Foreign trade taxes & 900 & 1,150 & 853 & 964 & 964 & 1,070 & 1,181 & 1,314 & 1,451 \\
\hline Other taxes & 368 & 424 & 420 & 411 & 411 & 506 & 562 & 633 & 678 \\
\hline Social fund contributions & 4,366 & 5,430 & 5,468 & 5,911 & 5,731 & 6,325 & 7,040 & 7,866 & 8,672 \\
\hline Health fund contributions & 841 & 1,157 & 1,327 & 1,479 & 1,449 & 1,583 & 1,740 & 1,906 & 2,081 \\
\hline Non-tax revenues & 1,628 & 1,575 & 979 & 1,121 & 1,121 & 1,141 & 1,254 & 1,374 & 1,500 \\
\hline Grants & 970 & 1,068 & 1,236 & 896 & 2,067 & 1,704 & 1,374 & 1,404 & 1,424 \\
\hline Budget support grants & 5 & 716 & 717 & 362 & 1,532 & 1,184 & 840 & 858 & 870 \\
\hline Foreign financed projects grants & 965 & 352 & 390 & 517 & 517 & 520 & 535 & 546 & 554 \\
\hline Revenues of special funds & 1,524 & 1,844 & 1,657 & 1,632 & 1,469 & 1,760 & 1,935 & 2,119 & 2,314 \\
\hline Expenditure and net lending & 22,416 & 26,147 & 27,837 & 32,588 & 29,579 & 30,632 & 32,223 & 35,267 & 38,168 \\
\hline Current expenditure & 18,467 & 21,693 & 24,738 & 28,734 & 25,725 & 25,855 & 26,971 & 29,096 & 31,433 \\
\hline Wages & 4,876 & 5,730 & 7,193 & 9,833 & 7,531 & 7,531 & 7,609 & 8,251 & 8,782 \\
\hline Goods and services & 4,656 & 5,838 & 6,249 & 6,755 & 6,290 & 6,236 & 6,673 & 7,237 & 7,900 \\
\hline Of which: health fund & 1,895 & 2,548 & 3,073 & 3,376 & 3,376 & 3,597 & 3,955 & 4,332 & 4,729 \\
\hline Interest payments & 635 & 733 & 848 & 808 & 808 & 914 & 853 & 801 & 766 \\
\hline Domestic & 435 & 569 & 640 & 472 & 472 & 589 & 533 & 492 & 472 \\
\hline Foreign & 200 & 163 & 208 & 337 & 337 & 325 & 320 & 309 & 294 \\
\hline Transfers & 7,979 & 8,875 & 10,095 & 10,917 & 10,676 & 10,757 & 11,378 & 12,290 & 13,421 \\
\hline Transfers to economy & 1,769 & 1,681 & 1,299 & 1,402 & 1,161 & 1,122 & 1,209 & 1,298 & 1,417 \\
\hline Transfers to households & 6,210 & 7,193 & 8,796 & 9,515 & 9,515 & 9,635 & 10,168 & 10,992 & 12,004 \\
\hline Of which: social fund & 5,231 & 6,015 & 7,599 & 8,261 & 8,261 & 8,007 & 8,539 & 9,354 & 10,210 \\
\hline Other current expenditure & 322 & 517 & 354 & 420 & 420 & 417 & 458 & 517 & 564 \\
\hline Net lending & -83 & 36 & -40 & -86 & -86 & -78 & -85 & -94 & -102 \\
\hline Capital expenditure & 4,032 & 4,419 & 3,138 & 3,940 & 3,940 & 4,855 & 5,337 & 6,264 & 6,838 \\
\hline Statistical discrepancy & 21 & 0 & $\ldots$ & $\ldots$ & $\ldots$ & $\ldots$ & $\ldots$ & $\ldots$ & $\ldots$ \\
\hline Overall balance (cash) & -103 & -630 & $-5,368$ & $-8,992$ & $-4,474$ & $-3,446$ & $-2,284$ & $-1,224$ & -795 \\
\hline Primary balance (cash) & 532 & 103 & $-4,520$ & $-8,184$ & $-3,665$ & $-2,532$ & $-1,431$ & -423 & -29 \\
\hline Change in arrears $(+$, increase $)$ & 14 & 0 & 0 & 0 & 0 & 0 & 0 & 0 & 0 \\
\hline Financing & 103 & 630 & 5,368 & 8,992 & 4,474 & 3,446 & 2,284 & 1,224 & 795 \\
\hline Budget financing & -377 & 283 & 4,850 & 1,918 & 3,395 & 1,967 & 912 & -109 & -284 \\
\hline Central government & -301 & 104 & 4,152 & 1,580 & 3,057 & 1,885 & 822 & -199 & -371 \\
\hline Net domestic & -156 & -519 & 841 & 1,204 & 814 & 1,531 & 458 & -480 & -122 \\
\hline Net foreign (excl. project loans) $1 /$ & -345 & -313 & 3,212 & 77 & 1,943 & 24 & 6 & -206 & -249 \\
\hline Privatization & 200 & 936 & 100 & 300 & 300 & 330 & 358 & 486 & 0 \\
\hline Local governments & -21 & 343 & 250 & 75 & 75 & 82 & 90 & 90 & 87 \\
\hline Of which: privatization & -21 & 343 & 150 & 75 & 75 & 82 & 0 & 0 & 0 \\
\hline Social fund & 87 & -48 & 197 & 263 & 263 & 0 & 0 & 0 & 0 \\
\hline Health fund & -142 & -117 & 251 & 0 & 0 & 0 & 0 & 0 & 0 \\
\hline Project loans & 480 & 347 & 518 & 1,079 & 1,079 & 1,496 & 1,389 & 1,351 & 1,094 \\
\hline Financing gap/unidentified financing & 0 & 0 & 0 & 5,996 & 0 & 0 & 0 & 0 & 0 \\
\hline \multicolumn{10}{|l|}{ Memorandum items: } \\
\hline GDP & 53,430 & 62,922 & 59,493 & 64,310 & 64,310 & 69,357 & 76,247 & 83,523 & 91,169 \\
\hline
\end{tabular}

Sources: Moldovan authorities; and IMF staff estimates and projections.

1/ In 2010, includes US\$150 million direct budget support from the IMF. 
Table 3. Moldova: General Government Budget, 2007-14 (Concluded)

(Percent of GDP, unless otherwise indicated)

\begin{tabular}{|c|c|c|c|c|c|c|c|c|c|}
\hline \multirow{3}{*}{ Revenues and grants } & \multirow{3}{*}{$\begin{array}{r}2007 \\
\\
41.7 \\
\end{array}$} & \multirow{3}{*}{$\begin{array}{r}2008 \\
40.6\end{array}$} & \multirow{2}{*}{$\begin{array}{l}2009 \\
\text { Proj. }\end{array}$} & \multicolumn{2}{|c|}{2010} & \multirow{2}{*}{$\begin{array}{l}2011 \\
\text { Prog. }\end{array}$} & \multirow{2}{*}{$\begin{array}{l}2012 \\
\text { Prog. }\end{array}$} & \multirow{2}{*}{$\begin{array}{l}2013 \\
\text { Proj. }\end{array}$} & \multirow{2}{*}{$\begin{array}{l}2014 \\
\text { Proj. }\end{array}$} \\
\hline & & & & $\begin{array}{c}\text { Proj. } \\
\text { without } \\
\text { measures }\end{array}$ & Prog. & & & & \\
\hline & & & 37.8 & 36.7 & 39.0 & 39.2 & 39.3 & 40.8 & 41.0 \\
\hline Revenues & 37.1 & 35.9 & 32.9 & 32.8 & 33.5 & 34.2 & 34.9 & 36.5 & 36.9 \\
\hline Tax revenues & 34.0 & 33.4 & 31.3 & 31.0 & 31.8 & 32.6 & 33.3 & 34.9 & 35.2 \\
\hline Profit tax & 2.6 & 1.1 & 0.6 & 0.3 & 0.3 & 0.5 & 0.5 & 1.5 & 1.5 \\
\hline Personal income tax & 2.5 & 2.4 & 2.3 & 2.4 & 2.3 & 2.4 & 2.4 & 2.4 & 2.6 \\
\hline VAT & 14.2 & 14.5 & 12.4 & 12.5 & 12.7 & 13.2 & 13.7 & 14.1 & 14.2 \\
\hline Excises & 2.6 & 2.5 & 2.3 & 2.2 & 3.1 & 2.9 & 3.0 & 3.0 & 2.9 \\
\hline Foreign trade taxes & 1.7 & 1.8 & 1.4 & 1.5 & 1.5 & 1.5 & 1.5 & 1.6 & 1.6 \\
\hline Other taxes & 0.7 & 0.7 & 0.7 & 0.6 & 0.6 & 0.7 & 0.7 & 0.8 & 0.7 \\
\hline Social fund contributions & 8.2 & 8.6 & 9.2 & 9.2 & 8.9 & 9.1 & 9.2 & 9.4 & 9.5 \\
\hline Health fund contributions & 1.6 & 1.8 & 2.2 & 2.3 & 2.3 & 2.3 & 2.3 & 2.3 & 2.3 \\
\hline Non-tax revenues & 3.0 & 2.5 & 1.6 & 1.7 & 1.7 & 1.6 & 1.6 & 1.6 & 1.6 \\
\hline Grants & 1.8 & 1.7 & 2.1 & 1.4 & 3.2 & 2.5 & 1.8 & 1.7 & 1.6 \\
\hline Budget support grants & 0.0 & 1.1 & 1.2 & 0.6 & 2.4 & 1.7 & 1.1 & 1.0 & 1.0 \\
\hline Foreign financed projects grants & 1.8 & 0.6 & 0.7 & 0.8 & 0.8 & 0.8 & 0.7 & 0.7 & 0.6 \\
\hline Revenues of special funds & 2.9 & 2.9 & 2.8 & 2.5 & 2.3 & 2.5 & 2.5 & 2.5 & 2.5 \\
\hline Expenditure and net lending & 42.0 & 41.6 & 46.8 & 50.7 & 46.0 & 44.2 & 42.3 & 42.2 & 41.9 \\
\hline Current expenditure & 34.6 & 34.5 & 41.6 & 44.7 & 40.0 & 37.3 & 35.4 & 34.8 & 34.5 \\
\hline Wages & 9.1 & 9.1 & 12.1 & 15.3 & 11.7 & 10.9 & 10.0 & 9.9 & 9.6 \\
\hline Goods and services & 8.7 & 9.3 & 10.5 & 10.5 & 9.8 & 9.0 & 8.8 & 8.7 & 8.7 \\
\hline Interest payments & 1.2 & 1.2 & 1.4 & 1.3 & 1.3 & 1.3 & 1.1 & 1.0 & 0.8 \\
\hline Domestic & 0.8 & 0.9 & 1.1 & 0.7 & 0.7 & 0.0 & 0.7 & 0.6 & 0.5 \\
\hline Foreign & 0.4 & 0.3 & 0.3 & 0.5 & 0.5 & 0.0 & 0.4 & 0.4 & 0.3 \\
\hline Transfers & 14.9 & 14.1 & 17.0 & 17.0 & 16.6 & 15.5 & 14.9 & 14.7 & 14.7 \\
\hline Transfers to economy & 3.3 & 2.7 & 2.2 & 2.2 & 1.8 & 1.6 & 1.6 & 1.6 & 1.6 \\
\hline Transfers to households & 11.6 & 11.4 & 14.8 & 14.8 & 14.8 & 13.9 & 13.3 & 13.2 & 13.2 \\
\hline Of which: social insurance fund & 9.8 & 9.6 & 12.8 & 12.8 & 12.8 & 11.5 & 11.2 & 11.2 & 11.2 \\
\hline Other current expenditure & 0.6 & 0.8 & 0.6 & 0.7 & 0.7 & 0.6 & 0.6 & 0.6 & 0.6 \\
\hline Net lending & -0.2 & 0.1 & -0.1 & -0.1 & -0.1 & -0.1 & -0.1 & -0.1 & -0.1 \\
\hline Capital expenditure & 7.5 & 7.0 & 5.3 & 6.1 & 6.1 & 7.0 & 7.0 & 7.5 & 7.5 \\
\hline Statistical discrepancy & 0.0 & 0.0 & $\ldots$ & $\ldots$ & $\ldots$ & $\ldots$ & $\ldots$ & $\ldots$ & $\ldots$ \\
\hline Overall balance (cash) & -0.2 & -1.0 & -9.0 & -14.0 & -7.0 & -5.0 & -3.0 & -1.5 & -0.9 \\
\hline Primary balance (cash) & 1.0 & 0.2 & -7.6 & -12.7 & -5.7 & -3.7 & -1.9 & -0.5 & 0.0 \\
\hline Change in arrears $(+$, increase $)$ & 0.0 & 0.0 & 0.0 & 0.0 & 0.0 & 0.0 & 0.0 & 0.0 & 0.0 \\
\hline Financing & 0.2 & 1.0 & 9.0 & 14.0 & 7.0 & 5.0 & 3.0 & 1.5 & 0.9 \\
\hline Budget financing & -0.7 & 0.4 & 8.2 & 3.0 & 5.3 & 2.8 & 1.2 & -0.1 & -0.3 \\
\hline Central government & -0.6 & 0.2 & 7.0 & 2.5 & 4.8 & 2.7 & 1.1 & -0.2 & -0.4 \\
\hline Net domestic & -0.3 & -0.8 & 1.4 & 1.9 & 1.3 & 2.2 & 0.6 & -0.6 & -0.1 \\
\hline Net foreign (excl. project loans) 1/ & -0.6 & -0.5 & 5.4 & 0.1 & 3.0 & 0.0 & 0.0 & -0.2 & -0.3 \\
\hline Privatization & 0.4 & 1.5 & 0.2 & 0.5 & 0.5 & 0.5 & 0.5 & 0.6 & 0.0 \\
\hline Local governments & 0.0 & 0.5 & 0.4 & 0.1 & 0.1 & 0.1 & 0.1 & 0.1 & 0.1 \\
\hline Of which: privatization & 0.0 & 0.5 & 0.4 & 0.1 & 0.1 & 0.1 & 0.1 & 0.1 & 0.1 \\
\hline Social fund & 0.2 & -0.1 & 0.3 & 0.4 & 0.4 & 0.0 & 0.0 & 0.0 & 0.0 \\
\hline Health fund & -0.3 & -0.2 & 0.4 & 0.0 & 0.0 & 0.0 & 0.0 & 0.0 & 0.0 \\
\hline Project loans & 0.9 & 0.6 & 0.9 & 1.7 & 1.7 & 2.2 & 1.8 & 1.6 & 1.2 \\
\hline Financing gap/unidentified financing & 0.0 & 0.0 & 0.0 & 9.3 & 0.0 & 0.0 & 0.0 & 0.0 & 0.0 \\
\hline Stock of public and publicly guaranteed debt & 26.8 & 21.3 & 30.9 & 36.6 & 36.9 & 39.6 & 41.0 & 38.6 & 36.0 \\
\hline Domestic debt & 7.0 & 5.6 & 8.6 & 7.9 & 8.3 & 7.4 & 6.4 & 5.6 & 4.9 \\
\hline Domestic expenditure arrears & 0.5 & 0.0 & 0.0 & 0.0 & 0.0 & 0.0 & 0.0 & 0.0 & 0.0 \\
\hline External debt & 19.8 & 15.7 & 22.4 & 28.6 & 28.6 & 32.3 & 34.5 & 33.0 & 31.1 \\
\hline \multicolumn{10}{|l|}{ Memorandum items: } \\
\hline GDP (millions of Moldovan lei) & 53,430 & 62,922 & 59,493 & 64,310 & 64,310 & 69,357 & 76,247 & 83,523 & 91,169 \\
\hline
\end{tabular}

Sources: Moldovan authorities; and IMF staff estimates and projections.

1/ In 2010, includes US\$150 million direct budget support from the IMF. 
Table 4. Moldova: Accounts of the National Bank of Moldova and Monetary Survey, 2007-10

(Millions of Moldovan lei, unless otherwise indicated)

\begin{tabular}{|c|c|c|c|c|c|c|c|c|}
\hline & \multirow[t]{3}{*}{2007} & \multirow[t]{3}{*}{2008} & \multicolumn{2}{|c|}{2009} & \multicolumn{4}{|c|}{2010} \\
\hline & & & Q3 & Q4 & Q1 & Q2 & Q3 & Q4 \\
\hline & & & & Proj. & Prog. & Prog. & Prog. & Prog. \\
\hline \multicolumn{9}{|l|}{ National Bank of Moldova } \\
\hline Net foreign assets & 13,254 & 15,650 & 10,896 & 16,014 & 16,850 & 17,753 & 18,342 & 18,488 \\
\hline NFA (convertible) & 13,280 & 15,655 & 10,906 & 16,023 & 16,860 & 17,763 & 18,352 & 18,498 \\
\hline Gross reserves & 14,869 & 17,393 & 14,848 & 17,905 & 19,116 & 20,224 & 21,103 & 21,862 \\
\hline Reserve liabilities & $-1,816$ & $-1,738$ & $-3,942$ & $-1,882$ & $-2,256$ & $-2,462$ & $-2,751$ & $-3,365$ \\
\hline Net domestic assets & $-3,717$ & $-4,016$ & $-1,352$ & $-5,114$ & $-6,066$ & $-6,864$ & $-7,342$ & $-6,772$ \\
\hline Net domestic assets at program exchange rates & $-5,301$ & $-8,720$ & $-1,491$ & $-5,090$ & $-5,834$ & $-6,411$ & $-6,661$ & $-5,867$ \\
\hline Net claims on general government & -92 & -479 & 948 & -762 & -269 & -306 & 92 & 1,205 \\
\hline Credit to banks & $-3,014$ & $-4,183$ & $-1,479$ & $-1,789$ & $-2,961$ & $-3,437$ & $-4,013$ & $-4,251$ \\
\hline Other items (net) & -610 & 646 & -822 & $-2,563$ & $-2,836$ & $-3,121$ & $-3,420$ & $-3,726$ \\
\hline Reserve money & 9,537 & 11,634 & 9,544 & 10,900 & 10,784 & 10,889 & 10,999 & 11,716 \\
\hline Currency in circulation & 6,665 & 7,551 & 7,258 & 8,284 & 8,196 & 8,276 & 8,360 & 8,904 \\
\hline Banks' reserves & 2,872 & 4,079 & 2,284 & 2,616 & 2,588 & 2,613 & 2,640 & 2,812 \\
\hline Required reserves & 1,998 & 2,896 & 994 & 1,140 & 1,262 & 1,218 & 1,195 & 1,316 \\
\hline Excess reserves & 874 & 1,183 & 1,290 & 1,476 & 1,326 & 1,395 & 1,445 & 1,496 \\
\hline \multicolumn{9}{|l|}{ Monetary survey } \\
\hline Net foreign assets & 11,705 & 12,540 & 11,032 & 15,038 & 14,569 & 15,222 & 15,547 & 15,448 \\
\hline NFA (convertible) & 11,855 & 12,746 & 11,174 & 15,230 & 14,755 & 15,417 & 15,746 & 15,645 \\
\hline Of which: commercial banks & $-1,425$ & $-2,909$ & 268 & -793 & $-2,104$ & $-2,346$ & $-2,606$ & $-2,852$ \\
\hline Foreign assets of commercial banks & 2,370 & 2,573 & 5,176 & 4,454 & 3,607 & 3,633 & 3,644 & 3,671 \\
\hline Foreign liabilities of commercial banks & $-3,796$ & $-5,482$ & $-4,908$ & $-5,247$ & $-5,711$ & $-5,979$ & $-6,250$ & $-6,523$ \\
\hline NFA (non-convertible) & -150 & -206 & -141 & -192 & -186 & -195 & -199 & -198 \\
\hline Net domestic assets & 15,639 & 19,141 & 18,546 & 16,572 & 18,310 & 17,444 & 17,121 & 19,115 \\
\hline Net claims on general government & 578 & -109 & 2,487 & 1,728 & 1,998 & 1,739 & 1,913 & 2,804 \\
\hline Credit to economy & 20,884 & 25,123 & 23,505 & 23,332 & 25,261 & 25,118 & 25,292 & 25,872 \\
\hline Moldovan lei & 11,769 & 14,780 & 13,260 & 12,379 & 14,175 & 13,899 & 13,941 & 14,388 \\
\hline Foreign exchange & 9,115 & 10,343 & 10,245 & 10,953 & 11,086 & 11,219 & 11,351 & 11,484 \\
\hline Other items (net) & $-5,823$ & $-5,873$ & $-7,446$ & $-7,205$ & $-8,488$ & $-8,949$ & $-9,412$ & $-10,084$ \\
\hline Broad money (M3) & 27,344 & 31,681 & 29,579 & 31,610 & 32,879 & 32,667 & 32,668 & 34,563 \\
\hline Broad money (M2: excluding foreign currency deposits) & 18,397 & 21,774 & 18,427 & 19,687 & 20,812 & 20,455 & 20,312 & 22,062 \\
\hline Currency in circulation & 6,665 & 7,579 & 7,258 & 8,284 & 8,196 & 8,276 & 8,360 & 8,904 \\
\hline Total deposits & 20,679 & 24,102 & 22,321 & 23,326 & 24,684 & 24,391 & 24,309 & 25,658 \\
\hline Domestic currency deposits & 11,714 & 14,179 & 11,166 & 11,403 & 12,616 & 12,179 & 11,953 & 13,158 \\
\hline Foreign currency deposits & 8,947 & 9,907 & 11,152 & 11,923 & 12,067 & 12,212 & 12,356 & 12,501 \\
\hline \multicolumn{9}{|l|}{ Memorandum items: } \\
\hline Reserve money growth (percent change, annual) & 46.4 & 22.0 & -15.3 & -6.3 & 22.1 & 14.5 & 15.3 & 7.5 \\
\hline Broad money growth (percent change, annual) & 39.8 & 15.9 & -8.6 & -0.2 & 16.9 & 12.4 & 10.4 & 9.3 \\
\hline Credit to economy (percent change, annual) & 51.7 & 20.3 & -7.5 & -7.1 & 2.4 & 5.3 & 7.6 & 10.9 \\
\hline Gross international reserves (millions of U.S. dollars) & 1,334 & 1,672 & 1,317 & 1,456 & 1,536 & 1,605 & 1,656 & 1,695 \\
\hline Percent of domestic-currency broad money & 81 & 80 & 81 & 91 & 92 & 99 & 104 & 99 \\
\hline Net international reserves (millions of U.S. dollars) & 1,173 & 1,505 & 980 & 1,303 & 1,354 & 1,410 & 1,440 & 1,434 \\
\hline $\begin{array}{l}\text { Net international reserves at program exchange rate (millions } \\
\text { of U.S. dollars) }\end{array}$ & $\ldots$ & $\ldots$ & 980 & 1,300 & 1,351 & 1,407 & 1,436 & 1,430 \\
\hline Broad money multiplier & 2.9 & 2.7 & 3.1 & 2.9 & 3.0 & 3.0 & 3.0 & 3.0 \\
\hline
\end{tabular}

Sources: National Bank of Moldova; and IMF staff projections. 
Table 5: Financial Sector Indicators, 2005-09

(End of period; percent, unless otherwise indicated)

\begin{tabular}{|c|c|c|c|c|c|c|}
\hline & 2005 & 2006 & 2007 & 2008 & 2009 & 2009 \\
\hline & & & & & June & Nov \\
\hline \multicolumn{7}{|l|}{ Size } \\
\hline Number of banks & 16 & 15 & 15 & 16 & 15 & 15 \\
\hline Total assets of the banking system (millions of Moldovan lei', & 17,939 & 22,749 & 31,979 & 39,122 & 36,587 & 38,103 \\
\hline Total loans of the banking system (percent of GDP) & 26.8 & 30.9 & 38.8 & 39.4 & 37.6 & 36.3 \\
\hline Total assets of the banking system (percent of GDP) & 47.6 & 50.8 & 59.9 & 62.2 & 61.5 & 64.0 \\
\hline \multicolumn{7}{|l|}{ Capital adequacy } \\
\hline Capital adequacy ratio $1 /$ & 27.2 & 27.9 & 29.1 & 32.2 & 32.8 & 32.7 \\
\hline \multicolumn{7}{|l|}{ Liquidity } \\
\hline Liquid assets (millions of Moldovan lei) & 6,609 & 7,625 & 9,296 & 11,983 & 11,510 & 13,553 \\
\hline Total Deposits (millions of Moldovan lei) & 13,235 & 17,243 & 23,086 & 27,196 & 24,183 & 25,031 \\
\hline Liquidity ratio $2 /$ & 48.0 & 44.2 & 40.3 & 44.1 & 47.6 & 54.1 \\
\hline Liquid assets as a share of total assets (percent) & 36.8 & 33.5 & 29.1 & 30.6 & 31.5 & 35.6 \\
\hline \multicolumn{7}{|l|}{ Asset quality } \\
\hline Gross loans (millions of Moldovan lei) & 10,078 & 13,830 & 20,753 & 24,772 & 22,379 & 21,625 \\
\hline Nonperforming loans (millions of Moldovan lei) & 535 & 595 & 768 & 1,276 & 2,343 & 3,594 \\
\hline Loan loss provisions & 529 & 707 & 873 & 1,247 & 1,471 & 1,888 \\
\hline Nonperforming loans as a share of total loans & 5.3 & 4.4 & 3.7 & 5.2 & 10.5 & 16.6 \\
\hline Loan-loss provisioning/nonperforming loans & 98.9 & 118.7 & 113.7 & 97.7 & 62.8 & 52.5 \\
\hline \multicolumn{7}{|l|}{ Profitability } \\
\hline Return on equity & 15.4 & 20.5 & 24.2 & 19.1 & 2.57 & 1.0 \\
\hline Return on assets & 3.2 & 3.4 & 3.9 & 3.4 & 0.5 & 0.2 \\
\hline \multicolumn{7}{|l|}{ Interest rates } \\
\hline Domestic currency average lending rate & 17.3 & 18.6 & 18.9 & 21.0 & 18.9 & 18.9 \\
\hline Domestic currency average deposit rate & 10.6 & 13.4 & 15.7 & 18.1 & 15.0 & 10.6 \\
\hline Interest rate spread, domestic currency & 6.8 & 5.2 & 3.2 & 2.9 & 3.9 & 8.3 \\
\hline Foreign currency average lending rate & 10.6 & 11.0 & 10.8 & 14.6 & 13.1 & 11.8 \\
\hline Foreign currency average deposit rate & 5.2 & 5.6 & 6.5 & 9.6 & 8.2 & 4.2 \\
\hline Interest rate spread, foreign currency & 5.5 & 5.4 & 4.3 & 5.0 & 4.9 & 7.6 \\
\hline 182-day T-bill (nominal yield) & 4.3 & 13.0 & 16.4 & 19.2 & 13.6 & 10 \\
\hline \multicolumn{7}{|l|}{ Foreign currency deposits and loans } \\
\hline Share of foreign currency denominated liabilities in total & $\ldots$ & $\ldots$ & 48.2 & 48.0 & 55.2 & 50.0 \\
\hline Share of foreign currency deposits in total deposits & 41.8 & 49.0 & 43.3 & 41.1 & 55.2 & 53.0 \\
\hline Share of foreign currency denominated loans in total loans & 37.4 & 38.2 & 43.6 & 41.2 & 44.4 & 44.3 \\
\hline
\end{tabular}

Source: National Bank of Moldova.

$1 /$ Total regulatory capital over total risk-weighted assets.

2/ Liquid assets over total deposits. 
Table 6. Moldova: External Financing Requirements and Sources, 2009-12

(Millions of U.S. Dollars, unless otherwise indicated)

\begin{tabular}{|c|c|c|c|c|}
\hline & 2009 & 2010 & 2011 & 2012 \\
\hline 1 Total requirements & 3,588 & 3,840 & 3,969 & 4,171 \\
\hline \multicolumn{4}{|c|}{ Current account deficit (excl. current transfers and compensation of } & 2,469 \\
\hline \multicolumn{5}{|l|}{ Of which } \\
\hline Exports of goods & 1,325 & 1,448 & 1,762 & 2,000 \\
\hline Imports of goods & $-3,100$ & $-3,357$ & $-3,821$ & $-4,174$ \\
\hline Debt amortization & 1,651 & 1,669 & 1,639 & 1,702 \\
\hline Public and publicly guaranteed & 49 & 46 & 44 & 46 \\
\hline Private & 1,602 & 1,623 & 1,595 & 1,656 \\
\hline 2 Identified financing sources & 3,371 & 3,559 & 3,767 & 4,041 \\
\hline Capital Account & -11 & -11 & -11 & -12 \\
\hline Foreign direct investment (net) & 91 & 197 & 259 & 295 \\
\hline Portfolio investment & -6 & -6 & -6 & -5 \\
\hline New borrowing & 1,817 & 1,774 & 1,783 & 1,847 \\
\hline Public & 195 & 26 & 2 & 2 \\
\hline Private & 1,622 & 1,749 & 1,781 & 1,846 \\
\hline Other capital flows & 37 & 100 & 80 & 80 \\
\hline Current transfers & 969 & 977 & 1,065 & 1,178 \\
\hline Worker's remittances & 646 & 700 & 759 & 855 \\
\hline Official transfers & 82 & 22 & 66 & 66 \\
\hline Other transfers & 241 & 255 & 240 & 257 \\
\hline Compensation of employees (income) & 489 & 536 & 604 & 675 \\
\hline Use of Fund credit & -15 & -9 & -6 & -16 \\
\hline Errors and omissions & -67 & 0 & 0 & 0 \\
\hline Exceptional financing & 69 & 0 & 0 & 0 \\
\hline 3 Gross international reserve accumulation (increase) & -217 & 239 & 180 & 214 \\
\hline 4 Financing gap & $\ldots$ & 521 & 383 & 344 \\
\hline Millions of SDR & $\ldots$ & 335 & 246 & 221 \\
\hline Percent of quota & $\ldots$ & 272 & 200 & 180 \\
\hline 5 Prospective financing & $\ldots$ & 521 & 383 & 344 \\
\hline IMF & $\ldots$ & 265 & 155 & 155 \\
\hline Millions of SDR & $\ldots$ & 170 & 120 & 80 \\
\hline Percent of quota & $\ldots$ & 138 & 97 & 65 \\
\hline Other donors & $\ldots$ & 256 & 228 & 189 \\
\hline European Commission & $\ldots$ & 114 & 115 & 86 \\
\hline World Bank & $\ldots$ & 113 & 60 & 60 \\
\hline EIB/EBRD/CEDB & $\ldots$ & 29 & 53 & 42 \\
\hline
\end{tabular}

Sources: Moldovan authorities; and IMF staff projections. 
Table 7. Moldova: Disbursements, Purchases, and Timing of Reviews Under the ECF/EFF Arrangements 1/

\begin{tabular}{|c|c|c|c|c|c|c|c|}
\hline \multirow[t]{2}{*}{ Date of availability } & \multirow[t]{2}{*}{ Conditions } & \multicolumn{3}{|c|}{ Amount (millions of SDRs) } & \multicolumn{3}{|c|}{ Percent of quota } \\
\hline & & Total & ECF & EFF & Total & ECF & EFF \\
\hline January 29, 2010 & Board approval of the arrangements & 60.00 & 40.00 & 20.00 & $48.70 \%$ & $32.5 \%$ & $16.2 \%$ \\
\hline June 30, 2010 & Observance of end-March 2010 performance criteria and completion of first review & 60.00 & 40.00 & 20.00 & $48.70 \%$ & $32.5 \%$ & $16.2 \%$ \\
\hline December 31, 2010 & Observance of end-September 2010 performance criteria and completion of second review & 50.00 & 40.00 & 10.00 & $40.58 \%$ & $32.5 \%$ & $8.1 \%$ \\
\hline June 30, 2011 & Observance of end-March 2011 performance criteria and completion of third review & 50.00 & 20.00 & 30.00 & $40.58 \%$ & $16.2 \%$ & $24.4 \%$ \\
\hline December 31, 2011 & Observance of end-September 2011 performance criteria and completion of fourth review & 50.00 & 16.96 & 33.04 & $40.58 \%$ & $13.8 \%$ & $26.8 \%$ \\
\hline June 30, 2012 & Observance of end-March 2012 performance criteria and completion of fifth review & 50.00 & 13.92 & 36.08 & $40.58 \%$ & $11.3 \%$ & $29.3 \%$ \\
\hline \multirow[t]{2}{*}{ December 31, 2012} & Observance of end-September 2012 performance criteria and completion of sixth review & 49.60 & 13.92 & 35.68 & $40.26 \%$ & $11.3 \%$ & $29.0 \%$ \\
\hline & Total: & 369.60 & 184.80 & 184.80 & $300.0 \%$ & $150.0 \%$ & $150.0 \%$ \\
\hline
\end{tabular}

Sources: IMF staff estimates and projections.

Note: A total of SDR 95 million of access under the ECF will be disbursed to the account of the Ministry of Finance at the National bank of Moldova for budget support. This amount will be spread over the first three purchases as follows: (i) SDR 40 million from the first purchase; (ii) SDR 40 million from the second purchase, and (iii) SDR 15 million from the third purchase.

1/ Moldova's quota is SDR 123.2 million 
Table 8. Moldova: Indicators of Fund Credit, 2008-18 1/

\begin{tabular}{|c|c|c|c|c|c|c|c|c|c|c|c|}
\hline & 2008 & 2009 & 2010 & 2011 & 2012 & 2013 & 2014 & 2015 & 2016 & 2017 & 2018 \\
\hline & Actual & \multicolumn{10}{|c|}{ Projection } \\
\hline \multicolumn{12}{|l|}{$\begin{array}{l}\text { Fund obligations based on existing credit } \\
\text { (millions of SDRs) }\end{array}$} \\
\hline Principal & 16.0 & 9.7 & 5.5 & 3.9 & 10.5 & 14.2 & 17.6 & 17.6 & 16.5 & 8.9 & 3.4 \\
\hline Charges and interest & 0.9 & 0.3 & 0.0 & 0.0 & 0.4 & 0.4 & 0.3 & 0.2 & 0.1 & 0.0 & 0.0 \\
\hline \multicolumn{12}{|l|}{ Fund obligations based on existing and prospective credit } \\
\hline Principal & 16.0 & 9.7 & 5.5 & 3.9 & 10.5 & 14.2 & 20.9 & 36.4 & 64.3 & 72.5 & 71.2 \\
\hline Charges and interest & 0.9 & 0.3 & 0.5 & 1.1 & 2.7 & 3.1 & 3.1 & 2.9 & 2.6 & 2.2 & 1.7 \\
\hline \multicolumn{12}{|l|}{ Total obligations based on existing and prospective credit } \\
\hline Millions of SDRs & 16.9 & 10.0 & 6.1 & 5.0 & 13.2 & 17.3 & 24.0 & 39.3 & 66.9 & 74.7 & 72.9 \\
\hline Millions of U.S. dollars & 26.7 & 15.3 & 9.5 & 7.7 & 20.6 & 26.8 & 37.2 & 60.9 & 103.7 & 115.7 & 112.9 \\
\hline Percent of exports of goods and services & 1.1 & 0.8 & 0.5 & 0.3 & 0.7 & 0.9 & 1.1 & 1.6 & 2.6 & 2.7 & 2.5 \\
\hline Percent of debt service $2 /$ & 32.7 & 17.8 & 10.8 & 9.2 & 20.5 & 23.9 & 31.0 & 36.7 & 40.1 & 37.5 & 33.7 \\
\hline Percent of GDP & 0.4 & 0.3 & 0.2 & 0.1 & 0.4 & 0.4 & 0.6 & 0.9 & 1.4 & 1.5 & 1.4 \\
\hline Percent of gross international reserves & 1.6 & 1.1 & 0.6 & 0.4 & 1.0 & 1.2 & 1.5 & 2.4 & 4.0 & 4.3 & 4.1 \\
\hline Percent of quota & 13.7 & 8.1 & 4.9 & 4.0 & 10.8 & 14.0 & 19.5 & 31.9 & 54.3 & 60.6 & 59.2 \\
\hline \multicolumn{12}{|l|}{ Outstanding Fund credit } \\
\hline Millions of SDRs & 107.9 & 98.2 & 262.6 & 358.7 & 447.8 & 433.6 & 412.7 & 376.2 & 311.9 & 239.4 & 168.3 \\
\hline Millions of U.S. dollars & 170.5 & 150.4 & 408.7 & 557.3 & 695.2 & 672.7 & 639.0 & 582.6 & 483.0 & 370.8 & 260.5 \\
\hline Percent of exports of goods and services & 6.9 & 7.9 & 19.6 & 22.2 & 24.6 & 21.4 & 18.4 & 15.5 & 12.0 & 8.6 & 5.7 \\
\hline Percent of debt service $2 /$ & 208.9 & 174.1 & 466.9 & 661.7 & 692.7 & 599.9 & 533.6 & 350.7 & 187.0 & 120.1 & 77.8 \\
\hline Percent of GDP & 2.8 & 2.8 & 8.0 & 10.5 & 12.2 & 11.0 & 9.7 & 8.3 & 6.5 & 4.7 & 3.1 \\
\hline Percent of gross international reserves & 10.2 & 10.3 & 24.1 & 29.7 & 33.3 & 30.0 & 26.2 & 22.7 & 18.6 & 13.9 & 9.4 \\
\hline Percent of quota & 87.6 & 79.7 & 213.2 & 291.2 & 363.5 & 352.0 & 335.0 & 305.4 & 253.2 & 194.4 & 136.6 \\
\hline Net use of Fund credit (millions of SDRs) & 6.9 & -9.7 & 164.5 & 96.1 & 89.1 & -14.2 & -20.9 & -36.4 & -64.3 & -72.5 & -71.2 \\
\hline Disbursements and purchases & 22.9 & 0.0 & 170.0 & 100.0 & 99.6 & 0.0 & 0.0 & 0.0 & 0.0 & 0.0 & 0.0 \\
\hline Repayments and repurchases & 16.0 & 9.7 & 5.5 & 3.9 & 10.5 & 14.2 & 20.9 & 36.4 & 64.3 & 72.5 & 71.2 \\
\hline \multicolumn{12}{|l|}{ Memorandum items: } \\
\hline Exports of goods and services (millions of U. S. dollars) & $2,483.2$ & $1,907.2$ & $2,080.4$ & $2,506.4$ & $2,831.7$ & $3,149.4$ & $3,476.7$ & $3,751.2$ & $4,019.1$ & $4,306.1$ & $4,590.9$ \\
\hline Debt service (millions of U. S. dollars) 2/ & 81.6 & 86.4 & 87.5 & 84.2 & 100.4 & 112.1 & 119.8 & 166.1 & 258.3 & 308.6 & 334.7 \\
\hline Nominal GDP (millions of U.S. dollars) 2/ & $6,054.8$ & $5,359.0$ & $5,094.8$ & $5,330.4$ & $5,705.7$ & $6,119.2$ & $6,581.8$ & $6,982.5$ & $7,407.0$ & $7,857.4$ & $8,335.1$ \\
\hline Gross International Reserves (millions of U.S. dollars) & $1,672.4$ & $1,455.7$ & $1,695.0$ & $1,875.2$ & $2,089.4$ & $2,245.3$ & $2,443.5$ & $2,563.6$ & $2,596.3$ & $2,660.2$ & $2,773.8$ \\
\hline Average exchange rate: SDR/US\$ & 0.6 & 0.7 & 0.6 & 0.6 & 0.6 & 0.6 & 0.6 & 0.6 & 0.6 & 0.6 & 0.6 \\
\hline Quota (millions of SDRs) & 123.2 & 123.2 & 123.2 & 123.2 & 123.2 & 123.2 & 123.2 & 123.2 & 123.2 & 123.2 & 123.2 \\
\hline
\end{tabular}

Sources: IMF staff estimates and projections.

1/ Assumes prospective disbursements of SDR 120 million in 2010, SDR 36.96 million in 2011, and SDR 27.84 million in 2012 under the ECF and purchases of SDR 50 million in 2010, SDR 66.04 million in 2011, and SDR 71.76 in 2012 under the EFF.

2/ Total debt service includes IMF repurchases and repayments. 
Table 9: Moldova: Localized Millennium Development Goals

\begin{tabular}{|c|c|c|c|c|c|c|c|c|c|}
\hline & \multirow[b]{2}{*}{2002} & \multirow[b]{2}{*}{2003} & \multirow[b]{2}{*}{2004} & \multirow[b]{2}{*}{2005} & \multirow[b]{2}{*}{2006} & \multirow[b]{2}{*}{2007} & \multirow[b]{2}{*}{2008} & \multicolumn{2}{|c|}{ Targets } \\
\hline & & & & & & & & 2010 & 2015 \\
\hline \multicolumn{10}{|l|}{ Goal: Reduce extreme poverty and hunger } \\
\hline - Population with consumption below $\$ 4.3$ (PPP) a day (percent) 1/ & $\ldots$ & $\ldots$ & $\ldots$ & $\ldots$ & 34.5 & 29.8 & 30.4 & 29.0 & 23.0 \\
\hline - Proportion of people under the absolute poverty line $1 /$ & 40.4 & 29.0 & 26.5 & 29.1 & 30.2 & 25.8 & 26.4 & 25.0 & 20.0 \\
\hline - Proportion of people under the extreme poverty line $1 /$ & 26.2 & 15.0 & 14.7 & 16.1 & 4.5 & 2.8 & 3.2 & 4.0 & 3.5 \\
\hline \multicolumn{10}{|l|}{ Goal. Achieve universal access to general secondary education } \\
\hline - Gross enrollment ratio in general secondary education (percent) & 95.1 & 95.1 & 94.6 & 94.4 & 92.0 & 91.6 & 90.9 & 95.0 & 98.0 \\
\hline - Literacy rate for the $15-24$ year-old population $1 /$ & $\ldots$ & $\ldots$ & $\ldots$ & $\ldots$ & 99.6 & 99.6 & 99.6 & 99.5 & 99.5 \\
\hline - Enrollment rate for pre-school programs for 3-6 year-old children & 57.0 & 61.1 & 66.1 & 70.7 & 70.1 & 72.6 & 74.4 & 75.0 & 78.0 \\
\hline - Enrollment rate for pre-school programs for 6-7 year-old children & 66.5 & 78.8 & 69.1 & 75.6 & 81.7 & $\ldots$ & $\ldots$ & 95.0 & 98.0 \\
\hline \multicolumn{10}{|l|}{ Goal: Reduce child mortality } \\
\hline - Infant mortality rate (per 1,000 live births) 1/ & 14.7 & 14.4 & 12.2 & 12.4 & 11.8 & 11.3 & 12.2 & 16.3 & 13.2 \\
\hline - Under-five mortality rate (per 1,000) 1/ & 18.2 & 17.8 & 15.3 & 15.6 & 14.0 & 14.0 & 14.4 & 18.6 & 15.3 \\
\hline - Immunization, measles (percent of children under 2 years old) & 94.3 & 95.7 & 96.3 & 96.9 & 96.9 & 94.7 & 94.4 & $>96 \%$ & $>96 \%$ \\
\hline \multicolumn{10}{|l|}{ Goal: Improve maternal health protection } \\
\hline - Maternal mortality ratio (per 100,000 births) & 28.0 & 21.9 & 23.5 & 18.6 & 16.0 & 15.8 & 38.4 & 15.5 & 13.3 \\
\hline - Births attended by skilled health personnel (percent) & 99.1 & 99.4 & 99.4 & 99.5 & 99.6 & 99.5 & 99.5 & 99.0 & 99.0 \\
\hline \multicolumn{10}{|l|}{ Goal: Combat HIVIAIDS, tuberculosis and other diseases } \\
\hline - HIV/AIDS incidence (per 100,000 people) $2 /$ & 4.7 & 6.2 & 8.4 & 12.5 & 14.7 & 17.4 & 19.4 & 9.6 & 8.0 \\
\hline - HIV incidence among $15-24$ year-olds $2 /$ & 9.0 & 9.8 & 13.4 & 20.1 & 18.8 & 21.2 & 16.1 & 11.2 & 11.0 \\
\hline - Mortality rate associated with tuberculosis (deaths per 100,000 people) $2 /$ & 17.3 & 16.9 & 17.1 & 19.1 & 19.3 & 20.2 & 17.1 & 15.0 & 10.0 \\
\hline \multicolumn{10}{|l|}{ Goal: Ensure environmental sustainability } \\
\hline - Proportion of land areas covered by forest (percent) & 10.3 & 10.5 & 10.6 & 10.7 & 10.7 & 10.7 & 10.9 & 12.1 & 13.2 \\
\hline - Ratio of area protected to maintain biological diversity (percent) & 1.96 & 1.96 & 1.96 & 1.96 & 4.7 & 4.7 & 4.8 & 4.65 & 4.65 \\
\hline - Share of population with access to improved water sources (percent) & 38.5 & 39.7 & 44.5 & 45.0 & 46.0 & 47.0 & 53.0 & 59.0 & 65.0 \\
\hline - The share of population with access to sewage & 31.3 & 31.7 & 32.8 & 43.8 & 43.3 & 43.9 & 45.7 & 50.3 & 65.0 \\
\hline
\end{tabular}

Sources: Economic Growth and Poverty Reduction Strategy Paper (EGPRSP) 2004-06, EGPRSP Monitoring Unit

1/ The methodology was changed from 2006.

2/ Including data from left bank of river Nistru. 


\section{ATTACHMENT I: LETTER OF INTENT}

Chişinău, January 14, 2010

Mr. Dominique Strauss-Kahn

Managing Director

International Monetary Fund

$70019^{\text {th }}$ Street NW

Washington, DC 20431 USA

Dear Mr. Strauss-Kahn:

The attached Memorandum of Economic and Financial Policies (MEFP) lays out the economic objectives, policies, and measures of the Government of Moldova for 2010-12. These objectives and policies are consistent with our Economic Stabilization and Recovery Plan, our engagements with the European Union, and our National Development Strategy. Based on our balance of payments needs and the policies described in the MEFP we request the approval of a blend of two three-year Fund arrangements - the Extended Credit Facility (ECF) and the Extended Fund Facility (EFF) - in the amount of the equivalent of SDR 369,600 million (300 percent of quota) in total for the period January 2010 through January 2013.

The overarching objective of the Government and the National Bank of Moldova is to improve the well-being of the population by promoting sustainable growth and reducing poverty. To this end, the policies set forth in the attached memorandum aim at ensuring macroeconomic stability, growth recovery, and financial sector development, improving the business environment, including through reduction of the footprint of the state in the economy, and strengthening the social safety net. In addition, in consultation with the IMF, we will take additional measures that may become appropriate for reaching these objectives.

The Government believes that the policies set forth in the attached MEFP are adequate to achieve the objectives of its program, but it will take any further measures that may become appropriate for this purpose. The Moldovan authorities will consult with the Fund on the adoption of these measures, and in advance of revisions to the policies contained in the MEFP, in accordance with the Fund's policies on such consultation. We will provide the Fund with the information it requests for monitoring progress during program implementation. We will also consult the Fund on our economic policies after the expiration of the arrangement, in line with Fund policies on such consultations, while we have outstanding purchases in the upper credit tranches. 
Sincerely yours,

$\frac{/ \mathrm{s} /}{\text { Vladimir Filat }}$
Prime Minister
Government of the Republic of Moldova

$/ \mathrm{s} /$

Valeriu Lazăr

Deputy Prime Minister Minister of Economy

$\frac{/ \mathrm{s} /}{\text { Veaceslav Negruţa }}$
Minister
Ministry of Finance

$/ \mathrm{s} /$

Dorin Drăguţanu

Governor

National Bank of Moldova

Attachments: Memorandum of Economic and Financial Policies

Technical Memorandum of Understanding 


\title{
Attachment II: Memorandum of Economic And Financial Policies for 2010-12
}

\author{
January 14, 2010
}

\section{BACKGROUND}

1. During 2006-2008, Moldova's economy grew strongly, but showed signs of overheating. Growth averaged about 5 percent, and reached nearly 8 percent in 2008 , boosted in part by remittances and foreign investment. The strong domestic demand, however, kept inflation in double digits during most of this period, and the current account deficit reached 17-18 percent of GDP. While fiscal policy maintained a small budget deficit and kept public debt low within the framework of an economic program supported by a PRGF arrangement with the IMF, it did not build up sufficient fiscal buffers against growing macroeconomic imbalances.

2. The global economic crisis led to a sharp rebalancing of the economy, putting considerable strains on society. In the first half of 2009, weak demand in trading partners led to a severe downturn in exports and worker remittances. The balance of payments moved from a surplus of US\$144 million to a deficit of US\$556 million over the same period as foreign direct investment and other capital inflows fell dramatically. While GDP dropped by 7.8 percent, domestic demand declined even faster, and the current account contracted to 11.2 percent of GDP. Alongside, deflation pressures have emerged, with the 12-month inflation registering -0.7 percent in November. The poverty level and unemployment rose significantly.

3. The crisis and pre-election spending hikes resulted in a large fiscal gap. Over the first nine months, public revenue dropped over 10 percent relative to 2008 due mainly to a drop in VAT receipts, nontax revenue, and foreign trade taxes. At the same time, current fiscal expenditure increased by over 13 percent, driven by large increases in wage and pension commitments well above available budget resources in the run-up to the April elections. The fiscal deficit increased from 1 percent of GDP in 2008 to about 6 percent of GDP in January-September 2009, financed mainly by a drawdown of previously accumulated balances in budget accounts and heavy domestic borrowing.

\section{Facing sustained depreciation pressures, the NBM sold about a third of its} international reserves defending the leu in early 2009. The sales (over US\$500 million) also led to sharp withdrawal of liquidity, thereby tightening monetary conditions despite falling inflation. Moreover, despite some 8 percent depreciation in January-April, the exchange rate strengthened vis-à-vis trading partners, negatively affecting the competitiveness of Moldova's exports. Since May the foreign exchange market stabilized and the NBM replenished a fraction of its foreign reserves (about US\$290 million), cut its base rate from 11 to 5 percent and lowered reserve requirements from $171 / 2$ to 8 percent, bringing commercial banks lending rates down. Nonetheless, at 17-20 percent the lending 
rates remain very high in real terms, contributing - together with the depressed economic conditions - to a sharp drop in demand for credit. The supply of credit has also tightened, as banks seem to prefer to channel the released liquidity into T-bills.

5. While the financial system remains stable, the recession has taken its toll on credit quality, and one bank has been closed. The decline in credit and capital increases in various banks have brought the average risk-weighted capital adequacy ratio of the system to 32.7 percent in November 2009, well above the required minimum of 12 percent. Liquidity remains abundant as well. Stress tests conducted by the NBM confirm that most banks' portfolios are robust to various risks. However, the share of nonperforming loans in total loans increased by over 10 percentage points from the beginning of the year, amounting to 16.6 percent in November 2009, and one medium-size bank (Investprivatbank) became insolvent in June. The insolvency of Investprivatbank resulted from a number of factors, including high portfolio concentration in recession-hit sectors and a number of risk management irregularities not reported to the NBM. Although individual depositors were paid in full, the bank has yet to be resolved pending the results of the valuation of its portfolio and tangible assets.

6. The NBM has responded by stepping up supervision and regulation. Banks have been requested to undergo a diagnostic study performed by an independent reputable audit firm to assess the quality of their assets and review their risk management methods. The NBM has also initiated close and enhanced monitoring of bank activity on the basis of monthly bank reports on financial soundness indicators and stress tests. To reduce the risk related to banks' large exposures and exposures to related parties, the NBM amended the respective regulations, reducing the limit of net exposures related to any person or a group of persons from 25 to 15 percent of capital, and the gross exposure related to any affiliated person or a group of affiliated persons from 20 to 10 percent of capital. It has also advised banks to: (i) increase the level of transparency of the ownership structure of banks; (ii) put in place well-defined liquidity management policies; and (iii) undertake stress tests to identify potential vulnerabilities.

7. The impact of the crisis was exacerbated by delays in implementation of marketoriented reforms and properly targeted social safety nets for the poor. A number of sectors of the national economy were excessively regulated with high barriers to entry and low competition. Consequently, domestic prices vastly exceeded international prices on many consumer products. Utility tariffs generally remained well below cost-recovery levels, leading to substantial arrears and underinvestment in these sectors. The introduction of the targeted social assistance system was delayed, posing undue hardship to many vulnerable groups while the budget allocation for the existing "nominal" (category-based) compensation social assistance scheme was exhausted by mid- 2009 .

8. In response to the economic crisis, we have launched an Economic Stabilization and Recovery Plan, which puts forward strong measures intended to help enterprises and 
households overcome the economic crisis. The plan's measures, a large part of which are included in this Memorandum, aim to stabilize public finances, restart economic growth, and protect vulnerable households. We have already implemented some first steps, aimed at promoting a market-based economy and improving the business climate to build a sustainable foundation for strong economic growth:

- To create a modern European public administration, a series of reforms have been launched to streamline and enhance the efficiency of the civil service. Several agencies and ministries have been closed, consolidated in existing structures, or reorganized to enhance the capacity to provide high-quality service delivery while minimizing the burden imposed on the private sector.

- To provide a boost to trade and promote competition, we have removed many formal and informal export and import restrictions. Specifically, we removed restrictions on wine, grapes, and grain exports that account for a sizable part of our exports. We have also abolished mandatory certification of every single shipment of imports, which should help improve the availability and lower the costs of imported goods.

- Our plan is calibrated to alleviate the impact on the most vulnerable. We expect the economic crisis to impact poverty indicators significantly, especially since 30 percent of households are recipients of remittances, which are expected to decrease more than 35 percent in 2009. Under such conditions, our goal is to balance public finances while protecting the most vulnerable households and supporting economic recovery.

\section{Program ObJectives for 2010-12}

9. The program for which we request Fund assistance aims to restore fiscal and external sustainability, preserve financial stability, and support growth. To these ends, our macroeconomic policies are built around four pillars: (i) fiscal policies to reverse the structural deterioration in 2008-2009 over the next three years while safeguarding public investment and social spending priorities; (ii) flexible monetary and exchange rate policies to keep inflation under control, facilitate adjustment to shocks, and rebuild foreign reserves; (iii) ensuring financial stability by strengthening the legal framework for bank resolution and close monitoring of the financial sector, accompanied by swift actions if and when necessary; and (iv) structural reforms to unlock and raise the economy's potential.

\section{Starting from an unfavorable position in 2009, our program aims to restore}

fiscal sustainability by 2012. After our decisive actions to bring expenditure closer to available resources, the 2009 budget deficit would be limited to 9 percent of GDP. We intend to build on this effort, lowering the headline deficit to 3 percent in 2012. These targets will be achieved mainly by steady, reform-based reduction of current expenditure to levels that are in line with Moldova's resources, while public investment will be raised and the social 
safety net enhanced. Moreover, revenue measures to broaden the tax base and modernize tax administration should raise revenue and support the adjustment.

11. Our macroeconomic objectives for $\mathbf{2 0 1 0}$ are cautiously optimistic, influenced by the expected gradual recovery in our trading partners. We expect the recession to be over by the first quarter of 2010, and the annual growth rate to reach $1 \frac{1}{2}$ percent. Monetary policy would aim to achieve a 5 percent inflation rate by year-end, a level we believe is appropriate for Moldova given the relatively high inflation in some major trading partners. The current account deficit would widen modestly to 101/4 percent of GDP from 9 percent in 2009 as imports are boosted by higher prices of imported energy and a recovery in investment.

12. Over the medium term, we expect the economy to recover to its potential, while inflation is kept low and stable. Growth is expected to reach 5 percent by 2012, led by a rebound in investment and exports, with remittances slowly growing back to their 2008 level. Inflation should remain in mid-single digits, anchored by growing credibility and transparency of monetary policy. The current account deficit would stabilize around 10 percent of GDP - a level that should be readily financed by FDI and official assistance. It will be helped by the fiscal consolidation and improvements in competitiveness brought by structural reforms and increased exchange rate flexibility, while reserves should remain at adequate levels.

\section{POLICIES FOR 2010}

\section{A. Fiscal Policy}

13. We intend to reduce the general government deficit to 7 percent in 2010 mainly by rationalizing current expenditure. A key challenge is to rein in the ballooning wage bill in the budget sector that would have reached an unaffordable 15.3 percent of GDP in 2010 on unchanged policies; the measures listed below would limit it to 11.7 percent of GDP. A second important objective is to reduce spending on goods and services by about 0.7 percentage point of GDP. Finally, we will cut low-priority subsidies to the real sector by 0.4 percentage points of GDP. On the revenue side, we have raised indirect taxes to obtain an additional 1.1 percentage point of GDP, while the reduction in the budget sector wage bill would lead to a loss of 0.4 percentage points of GDP relative to unchanged policies. The main policy measures are listed below.

\section{Main measures to reduce spending:}

a. $\quad$ Amend the Budget System Wage Law 355 to modify wage increases that are unaffordable in the current environment: (i) postpone the 20 percent raise for civil servants, the military, and staff of defense, security, and public order bodies until 2012; (ii) reschedule the wage increases for education sector employees so as to be implemented more gradually over 2009-2011; (iii) postpone all kinds of wage 
increases scheduled for 2010 to $2012-13$. These measures would generate savings of 1.8 billion lei in 2010 ( 2.8 percent of GDP) relative to unchanged policies.

b. Freeze budgetary sector employment to 232,000 employees, cutting staff positions that are permanently vacant, and reduce the budgeted 2010 wage bill by three percent on account of temporary vacancies, for total savings of about 400 million lei (0.6 percent of GDP).

c. Continue implementation of a plan adopted by the previous government to optimize the number of employees in the budgetary sector. In 2010, we will eliminate at least 4,000 positions, which will cut wage costs by about 50 million lei for the year and about 100 million lei on a full-year basis.

d. Apply a 20 percent average reduction in budget allocations for low-priority goods and services; to avoid arrears, enhance controls of the procurement process with a view to prevent the assumption of unaffordable commitments.

e. Reduce agricultural subsidies to 350 million lei, which is 300 million lei $(0.4$ percent of GDP) less than the 2009 allocation.

\section{Main measures to enhance revenue:}

f. Raise excise tax rates on a number of products (including gasoline, diesel, tobacco products, liquor, cosmetics, luxury cars, etc.) closer to the levels of neighboring countries. This will increase revenue in 2010 by about 600 million lei ( 0.9 percent of GDP);

g. Raise the VAT rate on natural gas from 5 to 6 percent. Net of the effect of VAT regime simplifications in agriculture, the budget will gain about 135 million lei (0.2 percent of GDP);

To ensure adequate budget financing in 2010, we request that the equivalent of SDR 95 million (about US\$150 million) from the total access under the arrangement, split between the first three tranches as shown in the attached Table 1, be used for budget support. This request is motivated by Moldova's lack of access to international capital markets and the limited absorption capacity of the domestic market for government securities, already near saturation after the heavy domestic borrowing in 2009.

We will pass the amended 2009 budget and the 2010 budget, as well as the amendments to Budget System Wage Law 355 as prior actions to our request for an IMF arrangement. We stand ready to implement additional fiscal measures to achieve the targeted budget deficit in the event revenues deteriorate or financing will not come as expected. 
14. To facilitate the return to fiscal sustainability in the medium term, we will also adopt measures that will have an effect after 2010. Specifically,

a. We will reinstate the corporate income tax with a low single rate (10 percent) and a broad, uniform base across sectors and regions. In order not to raise the tax burden on companies before the economy recovers from the crisis, this measure will apply to income earned from 2012 onwards. We are announcing it now to give time to companies to prepare. We will need technical assistance from the IMF to ensure best practice in defining the tax base with a view to minimize the burden on investment activity while ensuring uniform treatment of various economic agents.

b. Within the context of our education reform, we will optimize the network of education institutions, closing in 2010 four vocational schools and freezing the enrollment in three pedagogical colleges (to be closed in 2012); moreover, we will advance the consolidation of financial autonomy of universities.

\section{B. Monetary and Exchange Rate Policy}

\section{Monetary policy will continue to focus on steering inflation toward the NBM} target, and there is scope for further easing in the short run. The recent drop in inflation provides an opportunity to keep it at single digits in the period ahead, thereby providing a nominal anchor for the economy. At the same time, the large undershooting of the NBM's 2009 inflation target amid signs of deflation is worrisome. On balance, also taking into account the possible risk to inflation stemming from the expected recovery of energy prices and demand, these trends suggest that some monetary policy easing would be desirable, provided it does not compromise the 2010 inflation objectives.

\section{More generally, there is a need for a clear, credible, and operational monetary} policy framework, which should be communicated to the public and supported by further capacity building. To this effect, by March 31, 2010 the NBM's Council of Administration will adopt such a framework along with an action plan for its implementation with well-defined objectives, responsibilities, and deadlines to guide the necessary operational and communication efforts. Meanwhile, the NBM will restart open market operations, work toward enhancing its forecasting and analytical capacity, and promote transparency through regular communication with the public regarding the objectives and instruments of monetary policy. Starting in January 2010, the NBM will publish quarterly reports providing analyses of monetary policy performance as well as forecasts of inflation and key macroeconomic indicators. The NBM and the NBS will agree on a methodology and division of responsibilities for compiling and publishing core inflation, so that regular publication of monthly core inflation could begin no later than January 2010.

17. Meanwhile, the NBM's intervention in the foreign exchange market will be aimed only at counteracting erratic fluctuations without resisting trends. Recent developments have underscored Moldova's vulnerability to external shocks, justifying 
occasional foreign exchange market intervention. However, resisting sustained trends should be avoided because it raises output and fiscal costs of economic adjustment and wastes reserves. In this context, the NBM will promote a policy of sufficiently flexible exchange rate and will use intervention as an exceptional tool in times of heightened volatility.

\section{Taking advantage of the easing of global financial tensions, the NBM is} replenishing its foreign exchange reserves. Specifically, net international reserves as defined in the TMU are being raised to US\$1300 million to maintain a comfortable buffer against external shocks (prior action). This will broadly correspond to a cover of $4 \frac{1}{2}$ months of 2010 imports in terms of gross reserves. This coverage-expressed in an already depressed level of imports - seems appropriate given the still high downside risks to external stability. The ensuing injection of liquidity is also in line with the goal of short-run monetary policy easing and the need to support bank credit to the real sector.

\section{Safeguarding Financial Stability}

\section{The NBM has identified the need for legal amendments to strengthen its ability} to resort to early intervention in problem banks and address possible legal challenges to such actions. To this end, by February 15, 2010 the Cabinet will approve and submit to parliament a set of amendments to the Law on Financial Institutions that will strengthen the tools available to resolve problem banks (structural benchmark). The amendments will, in particular:

- Introduce a framework for early intervention through official administration whereby the NBM can bring troubled banks under its control with the goal of rehabilitating or resolving them quickly and efficiently;

- $\quad$ Strengthen the framework for carrying out purchase and assumption transactions;

- $\quad$ To the extent not covered under existing legislation, authorize and regulate the use of bridge banks and other similar instruments as a resolution mechanism; and

- $\quad$ Provide for NBM oversight of both official administrators and liquidators.

In light of the existing regime, the authorities will also review, in collaboration with Fund staff, the risks of litigation that may diminish the effectiveness of the bank resolution measures. Where necessary and appropriate, the amendments will include measures balancing adequate accountability through judicial review with need for effective bank resolutions that help preserve financial stability and provide insured depositors with speedy access to their funds.

Before April 15, 2010, the Cabinet will approve and submit to parliament a set of amendments to the Law about guaranteeing the deposits of physical persons in the banking system (LGD) that provides depositors with early access to their deposit funds in the event of a bank failure (structural benchmark). The amendments will, in particular: 
- $\quad$ Authorize early payment to insured depositors by the Deposit Guarantee Fund (DGF) based on bank records rather than on a lengthy validation process by the liquidator;

- $\quad$ Authorize the use of DGF funds to facilitate a purchase and assumption transaction;

- $\quad$ Provide for coordination and information-sharing between the DGF and the NBM and ensure confidentiality of information by revising procedures on appointment and qualifications for membership on the DGF board.

\section{The NBM will continue to monitor closely bank liquidity and foreign exchange} activity. To this end, it will perform monthly stress testing of each bank and will assess the banks' internal control systems and capacity to manage risks. The results of the stress tests and the ongoing independent diagnostics study of banks' portfolios will be used to determine the need for capital injections so that banks' CAR remain safely above the statutory ratio of 12 percent throughout the course of the program. The NBM will collect and share with Fund staff the results of the ongoing diagnostic studies in all banks that use conservative methods of asset valuation previously advised by Fund staff (structural benchmark).

\section{Structural Reforms}

\section{We strongly believe that implementing substantial reforms to improve the} business climate will be crucial to secure an economic recovery. To stimulate the domestic and foreign investment, a massive simplification of business regulation will be a key priority for the new government. Specifically, the government will:

- drastically reduce the number of categories of goods subject to mandatory compliance certification and licensing requirements and other types of authorizations imposed on business activities;

- $\quad$ enforce the one-stop shop policy to facilitate relations between companies and government without the need to interact with other government agencies;

- $\quad$ modernize the legal framework for construction authorization to reduce the duration and number of procedures related to obtaining construction permits;

- $\quad$ amend legislation on joint stock companies to bring it in line with best practice in investor protection;

- $\quad$ reduce the tax reporting burden by promoting the use of electronic statements;

- $\quad$ simplify procedures for business registration and liquidation;

- $\quad$ eliminate duplication of information requirements imposed by public agencies;

- $\quad$ substantially improve the regulatory framework of the sanitary and veterinary services. 
22. As part of our continuing efforts to reform the civil service, we intend to introduce a new merit- and performance-based wage system for public sector employees. Given the current unemployment situation, the new system will be introduced in 2011 once the pressure of the crisis begins to unwind.

23. We intend to reform the social insurance system with a view to improve its financial sustainability. The policies to achieve these include: (i) from 2010, phasing out early retirement privileges for civil servants, judges, and prosecutors by raising retirement age by 6 months every year until they reach the regular retirement age; (ii) extending the requirement to pay social insurance contributions to all persons employed in Moldova in line with bilateral treaties; and (iii) improving in 2010 the mechanism for sick leave compensation to align it better with the incentives of employers and employees.

24. We believe that the elimination of quasi-fiscal costs in the utility sector continues to be a key priority. Heating tariffs in particular should not be subject to political influence and should instead reflect an accurate and transparent assessment of costs. Therefore, by end2009 we will amend current legislation to ensure depoliticization of the tariff setting for heating by moving this responsibility to the National Agency for Energy Regulation (ANRE) (prior action). The new tariffs for heating, to be set by ANRE by January 14, 2010 will cover amortization and all variable costs that are necessary to produce, transport, distribute, and supply heating; moreover, it will be legally binding (prior action). We will also take steps to ensure independence of the regulator from the executive by transferring to parliament the power to appoint the ANRE's council of administration and approve its budget.

\section{E. Protecting Low-Income Households}

25. To help mitigate the impact of the recession on the most vulnerable, we will strengthen the efforts to ensure full implementation of the new targeted social assistance scheme. By March 31, 2010 the cabinet will approve a plan for the speedy expansion of the new targeted social assistance system with a view to cover at least $2 / 3$ of all eligible recipients by end-2010 (adoption of the plan will be a structural benchmark). An intensive communication campaign is already underway to promote enrollment of eligible households into the new system. The Guaranteed Minimum Income will be substantially increased to the expected 2010 extreme poverty level and new norms for determining eligibility will be approved by the Cabinet by end-January 2010. The 2010 budget allocation for social assistance, including unemployment benefits, will be raised by 36 percent to 630 million lei to ensure full financing of the new system. To minimize overlap of payments, we have accelerated phasing out the old "nominal" (category-based) untargeted system, discontinuing registration of new entrants and freezing the amount of benefits paid from January 1, 2010. 


\section{F. Safeguards Assessment}

26. We recognize the importance of completing a safeguards assessment by the first review under the arrangement. The NBM commits to receiving a safeguards mission, and to provide that mission with all requested information without delay.

\section{G. Program Monitoring}

27. The program will be monitored through semi-annual reviews, prior actions, quantitative performance criteria and indicative targets, and structural benchmarks. The first review under the program is expected to be completed by June 30, 2010, and the second review_-by December 31, 2010. Quantitative performance criteria (set for March 31, 2010 and September 30, 2010) include: (i) a ceiling on the general government deficit; (ii) a ceiling on the NBM's net domestic assets; (iii) a floor on the NBM's net international reserves; (iv) a ceiling on contracting or guaranteeing of nonconcessional external debt of the general government; and (v) non-accumulation of external payment arrears (continuous). Indicative targets are set for (i) a ceiling on reserve money; (ii) a ceiling on domestic expenditure arrears of the general government; (iii) a ceiling on the general government wage bill, and (iv) a floor on priority social spending. The indicators set as performance criteria will also serve as indicative targets at end-June 2010 and end-December 2010. The phasing of purchases under the arrangement and the review schedule are set out in Table 1 of this memorandum; the quantitative targets for end-March 2010, end-June 2010, endSeptember 2010, and end-December 2010, and continuous performance criteria are set out in Table 2; and the prior actions and structural benchmarks are set out in Table 3. Prior actions and structural benchmarks for the second review will be specified at the time of the first review. The understandings regarding the quantitative performance criteria and the structural measures described in this memorandum are further specified in the TMU attached to this memorandum.

28. In addition to the policies outlined in the attached MEFP, the government stands ready to take additional policy measures as appropriate to ensure the attainment of these objectives. We will consult with the Fund on adoption of new measures and provide the Fund with the information it requests for monitoring progress during program implementation. We will also consult the Fund on our economic policies after the expiration of the arrangement, in line with Fund policies on such consultations. 
Table 1. Moldova: Disbursements, Purchases, and Timing of Reviews Under the ECF/EFF Arrangements 1/

\begin{tabular}{|c|c|c|c|c|c|c|c|}
\hline \multirow[t]{2}{*}{ Date of availability } & \multirow[t]{2}{*}{ Conditions } & \multicolumn{3}{|c|}{ Amount (millions of SDRs) } & \multicolumn{3}{|c|}{ Percent of quota } \\
\hline & & Total & ECF & EFF & Total & ECF & EFF \\
\hline January 29, 2010 & Board approval of the arrangements & 60.00 & 40.00 & 20.00 & $48.70 \%$ & $32.5 \%$ & $16.2 \%$ \\
\hline June 30, 2010 & Observance of end-March 2010 performance criteria and completion of first review & 60.00 & 40.00 & 20.00 & $48.70 \%$ & $32.5 \%$ & $16.2 \%$ \\
\hline December 31, 2010 & Observance of end-September 2010 performance criteria and completion of second review & 50.00 & 40.00 & 10.00 & $40.58 \%$ & $32.5 \%$ & $8.1 \%$ \\
\hline June 30, 2011 & Observance of end-March 2011 performance criteria and completion of third review & 50.00 & 20.00 & 30.00 & $40.58 \%$ & $16.2 \%$ & $24.4 \%$ \\
\hline December 31, 2011 & Observance of end-September 2011 performance criteria and completion of fourth review & 50.00 & 16.96 & 33.04 & $40.58 \%$ & $13.8 \%$ & $26.8 \%$ \\
\hline June 30, 2012 & Observance of end-March 2012 performance criteria and completion of fifth review & 50.00 & 13.92 & 36.08 & $40.58 \%$ & $11.3 \%$ & $29.3 \%$ \\
\hline \multirow[t]{2}{*}{ December 31, 2012} & Observance of end-September 2012 performance criteria and completion of sixth review & 49.60 & 13.92 & 35.68 & $40.26 \%$ & $11.3 \%$ & $29.0 \%$ \\
\hline & Total: & 369.60 & 184.80 & 184.80 & $300.0 \%$ & $150.0 \%$ & $150.0 \%$ \\
\hline
\end{tabular}

Sources: IMF staff estimates and projections.

Note: A total of SDR 95 million of access under the ECF will be disbursed to the account of the Ministry of Finance at the National bank of Moldova for budget support. This amount will be spread over the first three purchases as follows: (i) SDR 40 million from the first purchase; (ii) SDR 40 million from the second purchase, and (iii) SDR 15 million from the third purchase.

1/ Moldova's quota is SDR 123.2 million 
Table 2. Moldova: Quantitative Performance Criteria and Indicative Targets, September 2009-December 2010 (Cumulative from the beginning of calendar year; millions of Moldovan lei unless otherwise indicated)

\begin{tabular}{|c|c|c|c|c|c|c|}
\hline & \multicolumn{2}{|c|}{2009} & \multicolumn{4}{|c|}{2010} \\
\hline & Sept. 30 & Dec. 31 & March 31 & June 30 & Sept. 30 & Dec. 31 \\
\hline & \multicolumn{2}{|r|}{ Projected } & $\begin{array}{l}\text { Performance } \\
\text { criteria }\end{array}$ & Indicative & $\begin{array}{l}\text { Performance } \\
\text { criteria }\end{array}$ & Indicative \\
\hline \multicolumn{7}{|l|}{ 1. Quantitative performance criteria } \\
\hline Ceiling on the overall cash deficit of the general government $1 /$ & 2,880 & 5,368 & 1,161 & 2,254 & 3,308 & 4,474 \\
\hline Ceiling on net domestic assets of the NBM (stock) $2 / 3 /$ & $-1,491$ & $-5,090$ & $-5,834$ & $-6,411$ & $-6,661$ & $-5,867$ \\
\hline Floor on net international reserves of the NBM (stock, millions of U.S. dollars) 2/ 3/ & 980 & 1,300 & 1,351 & 1,407 & 1,436 & 1,430 \\
\hline $\begin{array}{l}\text { Ceiling on contracting or guaranteeing of non-concessional external debt of the gene } \\
\text { government (millions of U.S. dollars) } 2 /\end{array}$ & $\cdots$ & $\cdots$ & 65 & 125 & 125 & 125 \\
\hline \multicolumn{7}{|l|}{ 2. Continuous performance criteria } \\
\hline Ceiling on accumulation of external payment arrears (millions of U.S. dollars) 2/ & 0 & 0 & 0 & 0 & 0 & 0 \\
\hline \multicolumn{7}{|l|}{ 3. Indicative targets } \\
\hline Ceiling on reserve money (stock) $3 /$ & 9,544 & 10,900 & 10,784 & 10,889 & 10,999 & 11,716 \\
\hline Ceiling on change in domestic expenditure arrears of the general government 4/ & 454 & 0 & 0 & 0 & 0 & 0 \\
\hline Ceiling on the general government wage bill & 5,081 & 7,193 & 1,888 & 3,905 & 5,665 & 7,531 \\
\hline Floor on priority social spending of the general government $5 /$ & 6,369 & 8,779 & 2,161 & 4,504 & 7,005 & 9,317 \\
\hline \multicolumn{7}{|l|}{ Memorandum items: } \\
\hline EC Macro Financial Assistance budgetary grants (millions of euros) & 0 & 0 & 0 & 25 & 25 & 50 \\
\hline \multicolumn{7}{|l|}{ Official external budget support and project grants and loans from the European } \\
\hline Commission and the World Bank (millions of U.S. dollars) & 48 & 97 & 33 & 126 & 169 & 243 \\
\hline Foreign-financed project loans & 293 & 518 & 175 & 441 & 924 & 1117 \\
\hline
\end{tabular}

Sources: Moldovan authorities; and IMF staff estimates and projections.

1/ Adjusters apply to the ceiling on the overall cash deficit of the general government in accordance with TMU (TMU paragraphs 21-23)

2/ Program target based on the program exchange rates set in the TMU (TMU paragraph 3).

$3 /$ Adjusters apply to ceiling on net domestic assets, floor on net international reserves and ceiling on reserve money in accordance with TMU (TMU paragraphs 24-25).

4/ Definition specified in the TMU (TMU paragraph 17). Amounts shown at end-September and end-December 2009 refer to stocks at that time.

$5 /$ The priority social spending of the general government is defined as the sum of essential recurrent expenditures directed to social assistance (TMU paragraph 19). 
Table 3. Moldova: Prior Actions and Structural Benchmarks 1/

\begin{tabular}{llll}
\hline Measure & Due & Status & Objective \\
\hline
\end{tabular}

\section{Prior actions}

Parliamentary passage of an amended 2009 budget with a deficit target in line with the MEFP (\$10).

Parliamentary passage of a 2010 budget with a deficit target in line with the MEFP (\$13).

Parliamentary passage of amendments to Budget System Wage Law 355 to bring wages in line with the wage bill allocation in the 2010 budget and with the MEFP ( $(13)$

The NBM's net international reserves, as defined in the TMU, will amount to US $\$ 1300$ million by endDecember (\$18).

Parliamentary passage of legislation transfering to ANRE the authority to set tariffs for heat directly, rather than indirectly through the municipalities as at present (\24).

The new heating tariff, to be set by ANRE by January 14, 2010, will cover at least amortization and all variable costs and will be legally binding (\24).

\section{Structural Benchmarks}

Cabinet approval and submission to parliament of amendments to the Law on Financial Institutions to strengthen the tools available to resolve problem banks (П19).

The NBM will collect and share with Fund staff the results of the ongoing diagnostic studies in all banks that use conservative methods of asset valuation previously agreed with Fund staff (\20).

The Cabinet will adopt a plan for the speedy expansion of the new targeted social assistance system with a view to cover at least $2 / 3$ of all eligible recipients by end-2010 (\$25).

Cabinet approval and submission to parliament of amendments to the Law about guaranteeing the deposits of physical persons in the banking system that provides depositors with early access to their deposit funds in the event of a bank failure (โ19).
PA Done To bring budget allocations in line with available resources.

PA Done

To facilitate macroeconomic stability and mitigate rising fiscal sustainability risks.

PA Done

To ensure the credibility of the budget and rationalize spending on wages to increase resources available for investment and social protection.

PA

Done

To provide for an adequate level of reserves and enhance market confidence by offsetting in part the loss of reserves in early 2009 .

PA Done To depoliticize tariff setting and foster cost recovery in the district heating sector.

PA

Done

To alleviate fiscal risks stemming from inadequate tariffs. 


\section{ATtACHMENT III: TeChNiCAL Memorandum OF Understanding}

1. This Technical Memorandum of Understanding (TMU) defines the variables subject to quantitative targets (prior actions, performance criteria and indicative benchmarks) established in the Memorandum of Economic and Financial Policies (MEFP) and describes the methods to be used in assessing the program performance with respect to these targets.

\section{A. Quantitative Program Targets}

2. The program will be assessed through performance criteria and indicative targets. Performance criteria are set with respect to:

- $\quad$ the ceiling on the overall cash deficit of the general government;

- $\quad$ the ceiling on the net domestic assets (NDA) of the National Bank of Moldova (NBM);

- $\quad$ the floor on the net international reserves (NIR) of NBM;

- the ceiling on contracting or guaranteeing of nonconcessional external debt of the general government;

- the ceiling on accumulation of external payment arrears of the central government (continuous).

Indicative targets are set on:

- $\quad$ the ceiling on reserve money;

- the ceiling on change in domestic expenditure arrears of the general government;

- $\quad$ the ceiling on the general government wage bill;

- the floor on priority social spending of the general government.

\section{B. Program Assumptions}

3. For program monitoring purposes, U.S. dollar denominated components of the NBM balance sheet will be valued at program exchange rates. The program exchange rate of the Moldovan leu (MDL) to the U.S. dollar has been set at $12.3000 \mathrm{MDL} / \mathrm{US} \$$. Amounts denominated in other currencies will be converted for program purposes into U.S. dollar amounts using the cross rates as of end-September 2009 published on the IMF web site 
http://www.imf.org, including US\$/EUR $=1.4643, \mathrm{JPY} / \mathrm{US} \$=89.7700, \mathrm{CHF} / \mathrm{US} \$=1.0290$, $\mathrm{US} \$ / \mathrm{GBP}=1.6113, \mathrm{CNY} / \mathrm{US} \$=6.8290, \mathrm{SDR} / \mathrm{US} \$=0.631164$.

\section{Institutional Definitions}

4. The general government is defined as comprising the central government and local governments. The central government includes the state budget (including special funds and special means, as well as foreign-financed projects), state social insurance budget, and health insurance budget. The local governments include special funds and special means, as well as foreign-financed projects. No new special or extrabudgetary funds will be created during the program period. Excluded from this definition are any government-owned entities with a separate legal status.

\section{Program Definitions}

5. Net international reserves (NIR) of the NBM in convertible currencies are defined as gross reserves minus reserve liabilities in convertible currencies. For program monitoring purposes, gross reserves of the NBM are defined as monetary gold, holdings of SDRs, reserve position in the Fund, and holdings of foreign exchange in convertible currencies that are readily available and controlled by the NBM, including holdings of securities denominated in convertible currencies that are freely usable for settlement of international transactions, calculated using program assumptions on bilateral exchange rates. Excluded from reserve assets are capital subscriptions to foreign financial institutions, long-term nonfinancial assets, funds disbursed by the World Bank or other international institutions assigned for on-lending and project implementation, assets in nonconvertible currencies, and foreign assets pledged as collateral or otherwise encumbered, including claims in foreign exchange arising from transactions in derivative assets (futures, forwards, swaps, and options). Reserve liabilities in convertible currencies are defined as use of Fund credit by the NBM, and convertible currency liabilities of the NBM to nonresidents with an original maturity of up to and including one year. Liabilities arising from use of Fund credit by the NBM do not include liabilities arising from the use of SDR allocation and use of Fund credit by the general government. Excluded from reserve liabilities are liabilities with original maturities longer than one year.

6. Reserve money is defined as currency in circulation (outside banks), vault cash of banks, total required reserves, and balances on correspondent accounts of banks in the NBM in lei. For the purpose of assessing compliance with the program targets, the value of reserve money will be calculated as arithmetic average of its values for the last 5 working days before and including the program test date. 
7. Net foreign assets (NFA) of the NBM are defined as gross reserves in convertible currencies (defined in paragraph 5) plus foreign assets in nonconvertible currencies, funds disbursed by the World Bank or other international institutions assigned for on-lending and project implementation, and foreign assets pledged as collateral or otherwise encumbered, including claims in foreign exchange arising from transactions in derivative assets, and net other foreign assets, minus foreign exchange liabilities of the NBM to nonresidents.

8. Net domestic assets (NDA) of the NBM is defined as the difference between reserve money (defined in paragraph 6) and net foreign assets (NFA) of the NBM. For the purpose of assessing compliance with the program targets, the value of NDA will be calculated as arithmetic average of its values for the last 5 working days before and including the program test date.

9. For the purposes of calculating overall cash deficit of the general government, net credit of the banking system to the general government is defined as outstanding claims of the banking system on the general government (exclusive of the claims associated with accrued interest, tax and social contribution payments by commercial banks, and foreign financed on-lending by banks), including overdrafts, direct credit and holdings of government securities, less deposits of the general government (excluding accrued interest on government deposits, and including the accounts for foreign-financed projects). ${ }^{5}$ The Ministry of Finance will provide data on the holdings of government securities and foreignfinanced projects.

10. The ceilings on the overall cash deficit of the general government are cumulative from the beginning of calendar year and will be monitored from the financing side as the sum of net credit of the banking system to the general government, the general government's net placement of securities outside the domestic banking system, other net credit from the domestic non-banking sector to the general government, the general government's receipt of disbursements from external $\mathrm{debt}^{6}$ for direct budgetary support and for specific projects minus amortization paid, and privatization proceeds stemming from the sale of the general government's assets, after deduction of the costs directly associated with the sale of these assets.

\footnotetext{
${ }^{5}$ For the calculation of the net credit of the banking system to general government the following accounts will be excluded: 1731, 1732, 1733, 1735, 1761, 1762, 1763, 1801, 1802, 1805, 1807, 2711, 2717, 2721, 2727, 2732, 2733, 2796, 2801 and 2802.

${ }^{6}$ Debt is defined as in footnote 3 .
} 
11. Government securities in the form of coupon-bearing instruments sold at face value will be treated as financing items in the fiscal accounts, in the amount actually received from buyers. On redemption date, the sales value (face value) will be recorded as amortization, and the coupon payments will be recorded as domestic interest payments.

12. External debt ceilings apply to the contracting or guaranteeing by the general government or any other agency acting on behalf of the general government of (i) short-term external debt (with an original maturity of up to and including one year) and (ii) nonconcessional medium- and long-term debt with original maturities of more than one year. Debt denominated in currencies other than the U.S. dollar shall be converted to the U.S. dollars using program assumptions on bilateral exchange rates. The debt ceilings will not apply (i) to loans classified as international reserve liabilities of the NBM, (ii) to changes in indebtedness resulting from refinancing credits and rescheduling operations of existing debt, (iii) to credits from international financial institutions (IFIs), includng credits extended by the Fund.

13. For program purposes, the definition of debt is set forth in point No. 9 of the Guidelines on Performance Criteria with Respect to Foreign Debt (Decision No. 12274, adopted on August 24, 2000 and revised on August 31, 2009). ${ }^{7}$ This definition applies also to commitments contracted or guaranteed for which value has not been received, and to private debt for which official guarantees have been extended and which, therefore, constitute a contingent liability of the public sector. Excluded from this definition are normal importrelated credits, defined as liabilities that arise from the direct extension, during the normal course of trading, of credit from a supplier to a purchaser - that is, when payment of goods and services is made at a time that differs from the time when ownership of the underlying goods or services changes. Normal import credit arrangements covered by this exclusion are self-liquidating; they contain pre-specified limits on the amounts involved and the times at which payments must be made; they do not involve the issuance of securities.

14. For purpose of the program, the guarantee of a debt arises from any explicit legal obligation of the general government or the NBM or any other agency acting on behalf of the general government to service such a debt in the event of nonpayment by the recipient

\footnotetext{
${ }^{7}$ Debt is defined as a current, i.e., not contingent, liability, created under a contractual arrangement through the provision of value in the form of assets (including currency) or services, and which requires the obligor to make one or more payments in the form of assets (including currency) or services, at some future point(s) in time; these payments will discharge the principal and/or interest liabilities incurred under the contract. Arrears, penalties, and judicially awarded damages arising from the failure to make payment under a contractual obligation that constitutes debt are debt. Failure to make payment on an obligation that is not considered debt under this definition (e.g., payment on delivery) will not give rise to debt.
} 
(involving payments in cash or in kind), or from any implicit legal or contractual obligation to finance partially or in full any shortfall incurred by the debtor.

15. Concessionality will be calculated using currency-specific discount rates based on the OECD commercial interest reference rates (CIRRs) and taking into account all details of the loan agreement, inlcuding maturity, grace period, payment schedule, upfront commision, and management fees. The ten-year average of CIRRs will be used as the discount rate to assess the concessionality of loans of an original maturity of at least 15 years, and a sixmonth average of CIRRs will be used to assess the concessionality of loans with original maturities of less than 15 years. To both the ten-year and six-month averages, the following margins will be added: 0.75 percent for repayment periods of less than 15 years; 1 percent for 15-19 years; 1.15 percent for 20-30 years; and 1.25 percent for over 30 years. Grant element of the loan can be calculated using the concessionality calculator available at the IMF web site http://www.imf.org ${ }^{8}$ For program purposes, a debt is concessional if it includes a grant element of at least 35 percent, calculated as follows: the grant element of a debt is the difference between the present value (PV) of debt and its nominal value, expressed as a percentage of the nominal value of the debt. The PV of debt at the time of its contracting is calculated by discounting the future stream of payments of debt service due on this debt. The discount rates used for this purpose are the CIRRs published by the OECD.

16. For the purposes of the program, external payments arrears will consist of all overdue debt service obligations (i.e., payments of principal or interest) arising in respect of any debt contracted or guaranteed or assumed by the central government, or the NBM, or any agency acting on behalf of the central government. The ceiling on new external payments arrears shall apply on a continuous basis throughout the period of the arrangement. It shall not apply to external payments arrears arising from external debt being renegotiated with external creditors, including Paris Club creditors; and more specifically, to external payments arrears in respect of which a creditor has agreed that no payment needs to be made pending negotiations.

17. For the purposes of the program, general government expenditure arrears are defined as non-disputed (in or out of court) payment obligations that are due but not paid for more than 30 days. They can arise on any expenditure item, including transfers, debt service, wages, pensions, energy payments and goods and services. Arrears between the state, local government, and social and health insurance budgets, are not counted towards the expenditure arrears' ceiling on the general government.

\footnotetext{
${ }^{8}$ Currently available at http://www.imf.org/external/np/pdr/conc/calculator/default.aspx.
} 
18. The general government wage bill will be defined as sum of budget spending on wages and salaries of public sector employees. This will include current spending reported in line "Wages" of the general government budget according to the program classification of the annual budget except for salaries of the social and health funds' employees. ${ }^{9}$

19. The priority social spending of the general government is defined as the sum of essential recurrent expenditures for social assistance, unemployment insurance, and pension payments as well as 95 percent of health expenditures.

\section{E. Adjusters}

20. The adjusters set in this TMU apply for assessing compliance with the program's quantitative targets starting from end-March 2010. They will not apply for (i) assessing compliance with the prior actions for the Board consideration of the request for a new program arrangement and (ii) evaluating the actual outcome of 2009.

21. The ceiling on the overall cash deficit of the general government will be increased by the amount paid in cash for recapitalization of the NBM or by the face value of government securities issued for the same purpose.

22. The ceiling on the overall cash deficit of the general government will be adjusted upward (downward) - that is, the deficit target will be increased (reduced) — by the full amount of any shortfall (excess) between actually disbursed and programmed Macro Financial Assistance budgetary grants from the European Commission (EC).

23. The ceiling on the overall cash deficit will be adjusted downward (upward) - that is, the deficit target will be reduced (increased) - for any lower (higher) than programmed disbursement of foreign-financed project loans as specified in Table 2 of the MEFP. Owing to monitoring lags, the downward adjustment is capped at a quarter of the programmed amount of foreign-financed project loans. The upward adjustment is capped at the equivalent of US\$25 million, evaluated at program exchange rates.

24. The ceiling on reserve money will be adjusted downward (upward) and the ceiling on NDA of NBM will be adjusted downward (upward) symmetrically for any reduction (increase) in the required reserve ratio on the deposits of commercial banks denominated in lei. The adjustment amount will be calculated by multiplying the change in the required

\footnotetext{
${ }^{9}$ For the calculation of the total general government wage bill the following accounts for central government, local government, and special funds from the Treasury system in the Ministry of Finance will be used: 111, 112, and 116.
} 
reserve ratio by the amount of commercial banks' deposits and liabilities in lei subject to reserve requirements.

25. The floor on NIR of the NBM will be lowered and the ceiling on NDA of NBM will be raised symmetrically by any shortfall in the official external grants and loans from the EC and World Bank capped up to an equivalent of US\$50 million. For the purpose of this definition, the program exchange rates will apply for calculating the amounts of the grants and loans.

\section{F. Reporting Requirements}

26. Macroeconomic data necessary for assessing compliance with performance criteria and indicative targets and benchmarks will be provided to Fund staff including, but not limited to data as specified in Table 1 . The authorities will transmit promptly to Fund staff any data revisions. 
Table 1. Moldova: Data to be Reported to the IMF

\section{Item}

Fiscal data (to be provided by the MoF)

General budget operations for revenues, expenditure and financing (functional and economic)

General government wage bill

Number of budgetary sector positions by ministry

Number of employees in the budgetary sector by ministry, and their respective wage bill

Expenditure on social assistance as stipulated under activity 457 of social payments paid from the social fund budget

Expenditure on pensions and unemployment benefits, and health expenditures as reported by NSIH and NHIC respectively

Domestic debt

Domestic arrears

Privatization receipts received by the budget (in lei and foreign exchange, net of divestiture transactions costs)

Monetary data (to be provided by the NBM)

Monetary survey of the NBM

Monetary survey for the whole banking system

Net claims on general government (NBM and commercial banks)

Financial indicators of commercial banks (from NBM's

Banking Supervision)

Foreign exchange cash flows

Foreign exchange operations (NBM data)

Foreign exchange market data (volume of trades, interventions, exchange rates)

NBM's sterilization operations

Interbank transactions (volumes, average rates)

\section{Periodicity}

Monthly, within three weeks of the end of each month

Monthly, within three weeks of the end of each month

Monthly, within three weeks of the end of each month

Monthly, within three weeks of the end of each month

Monthly, within three weeks of the end of each month

Monthly, within three weeks of the end of each month

Monthly, within two weeks of the end of each month

Monthly, within three weeks of the end of each month

Monthly within three weeks of the end of each month

Weekly within one week of the end of each week

Weekly within two weeks of the end of each week

Weekly within two weeks of the end of each week

Monthly within four weeks of the end of each month

Monthly, within two weeks of the end of each month

Monthly, within two weeks of the end of each month

Daily within 12 hours of the end of each day

Weekly within one week of the end of each week

Weekly within one week of the end of each week 


\section{Table 1. Moldova: Data to be Reported to the IMF (continued)}

\section{Item}

Balance of Payments (to be provided by the NBM)

Current and capital account data.

Transfers of individuals from abroad through the banking system

External debt data (to be provided by MoF and NBM)

Information on all new external loans contracted by the government or government guarantee.

Total debt service due by creditor, and debt service paid.

Disbursements of grants and loans by creditor

Other data (to be provided by NBS)

Overall consumer price index.

National accounts by sector of production, in nominal and real terms.

Export and import data on value, volume, and unit values, by major categories and countries.

\section{Periodicity}

One quarter after the end of the previous quarter

Monthly within six weeks of the end of each month

Monthly within three weeks of the end of each month

Monthly within three weeks of the end of each month

Monthly, within three weeks of the end of each month

Monthly within two weeks of the end of each month.

Quarterly within three months of the end of each quarter.

Monthly within two months of the end of each month. 


\title{
INTERNATIONAL MONETARY FUND AND INTERNATIONAL DEVELOPMENT ASSOCIATION
}

\author{
REPUBLIC OF MOLDOVA
}

\section{Joint IMF-World Bank Debt Sustainability Analysis}
Prepared by the Staffs of the International Monetary Fund and the International Development Association

Approved by Adam Bennett and Christian Mumssen (IMF) and Carlos Primo Braga and Luca Barbone (IDA)

January 15,2010 


\section{DebT SuStainability ANALYSIS}

The joint IMF-World Bank low-income country debt sustainability analysis (LIC DSA) indicates that Moldova's risk of debt distress is low at present, but additional factors increase vulnerability compared to the previous DSA. Under the baseline scenario, the debt burden will temporarily increase in the medium term as a result of significant financing contracted by the government to counteract the effects of the economic crisis. The indicator of net present value (NPV) of the debt-to-GDP ratio could temporarily breach its indicative threshold under two of the conventional stress tests, but other indicators remain below their respective thresholds. However, large private sector debt and potential large borrowing on non-concessional terms signal elevated risks and warrant a continuing careful approach to external financing.

1. The DSA presented here reflects the macroeconomic framework underlying staff projections under the program supported by a blend of Extended Credit Facility Extended Fund Facility Arrangements (ECF/EFF) and extended until 2029. It assumes that the implementation of prudent macroeconomic and structural policies, including a fiscal framework that aims to reverse recent structural fiscal deterioration, and adoption of the flexible exchange rate policy, will help Moldova recover from the economic crisis and resume sustainable growth. ${ }^{10}$

\section{BACKGROUND}

\section{Reflecting strong growth, Moldova's} total external debt burden has declined in recent years, helped by the shrinking public debt. ${ }^{11}$ At end-2008 public debt was low and was mostly owed to multilaterals and Paris Club creditors on concessional terms, without significant rollover risks. The ratios of debt service to exports and to fiscal revenues more than halved since 2006 and remain reasonably

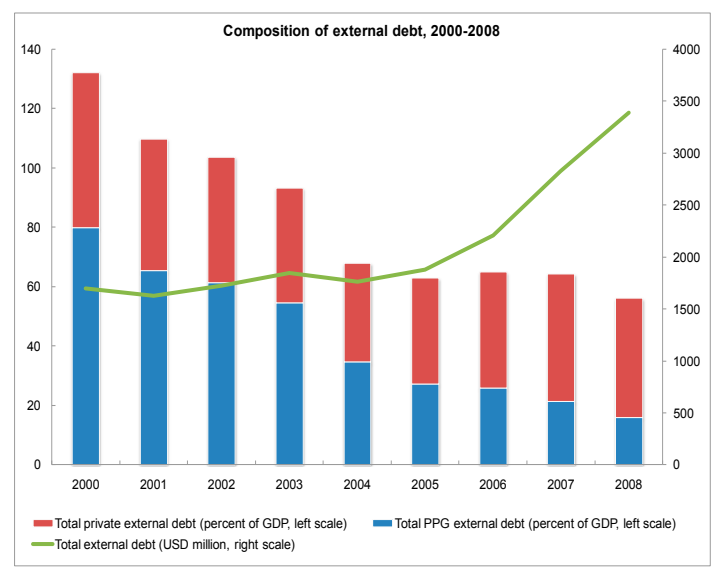

10 The DSA scenarios presented in this document were produced jointly by Fund and Bank staffs following "Staff Guidance Note on the Application of the Joint Fund-Bank Debt Sustainability Framework for LowIncome Countries (LICs)" of October 06, 2008, available at www.imf.org/external/np/pp/eng/2008/070308.pdf and http://siteresources.worldbank.org/INTDEBTDEPT/PolicyPapers/21952151/39748MainExt.pdf .

${ }^{11}$ Consistent with the definition of the general government in the Technical Memorandum of Understanding (TMU) for the new Fund-supported program, the public debt covers gross debt of the general government, while debts of state-owned enterprises are not included unless they are explicitly guaranteed by the government. In the absence of reliable data, both private and general government debt exclude liabilities of Transnistria, though press articles suggest large energy-related external arrears in that region. In line with the DSA guidelines, public debt includes liabilities towards the IMF. 
low. In the recent review of debt limits in Fund-supported programs, Moldova has been classified as a higher-capacity country, reflecting progress made in upgrading capacities for managing domestic and external public debt, reforming public administration, and improving transparency and accountability in the public sector. This progress is important, taking into account the significant borrowing that Moldova intends to contract from its international partners in the next few years.

\section{At the same time, private sector borrowing remains high.}

Between 2004 and 2008, the external private debt increased from 33 to 40 percent of GDP, two-and-a-half times the size of the public and publicly guaranteed (PPG) external debt. This increase was mainly caused by rising external exposure of the banking sector, both on a short-term (currency and deposits) and on a long-term (credits) basis. The majority of non-bank debt is short-term, and consists of trade credits, arrears and other payment liabilities, mostly for the imports of natural resources. The latter emerged in part as a result of heating tariffs set below cost recovery levels, which created a sequence of domestic and (later on) external payment arrears. This debt could potentially become a fiscal liability of up to 3 percent of GDP, since the heat production and distribution companies that are not paying for energy resources are publicly-owned companies.

\section{The long-term debts of the} non-banking sector are loans. Their share in total non-bank debt has been rising since 2005, reflecting mainly the increasing share of foreign-

\begin{tabular}{crrr}
\multicolumn{4}{c}{ Moldova: Composition of External PPG Debt, 2008} \\
\hline USD milion & Percent of GDP & $\begin{array}{r}\text { Net Persent Value in } \\
\text { USD million }\end{array}$ \\
\hline Total external debt & 3386 & 56 & $\ldots$ \\
External private debt & 2433 & 40 & $\ldots$ \\
External public debt & 953 & 16 & 742 \\
Multilaterals & 644 & 11 & 479 \\
IMF & 164 & 3 & 136 \\
World Bank & 436 & 7 & 307 \\
IDA & 311 & 5 & 184 \\
IBRD & 125 & 1 & 123 \\
Others & 44 & 5 & 36 \\
Official bilateral & 276 & 4 & 240 \\
Paris Club & 257 & 0 & 225 \\
Non-Paris Club & 19 & 1 & 16 \\
Commercial & 33 & & 23 \\
\hline
\end{tabular}

Source: Moldovan Ministry of Finance, National Bank of Moldova and Fund staff calculations
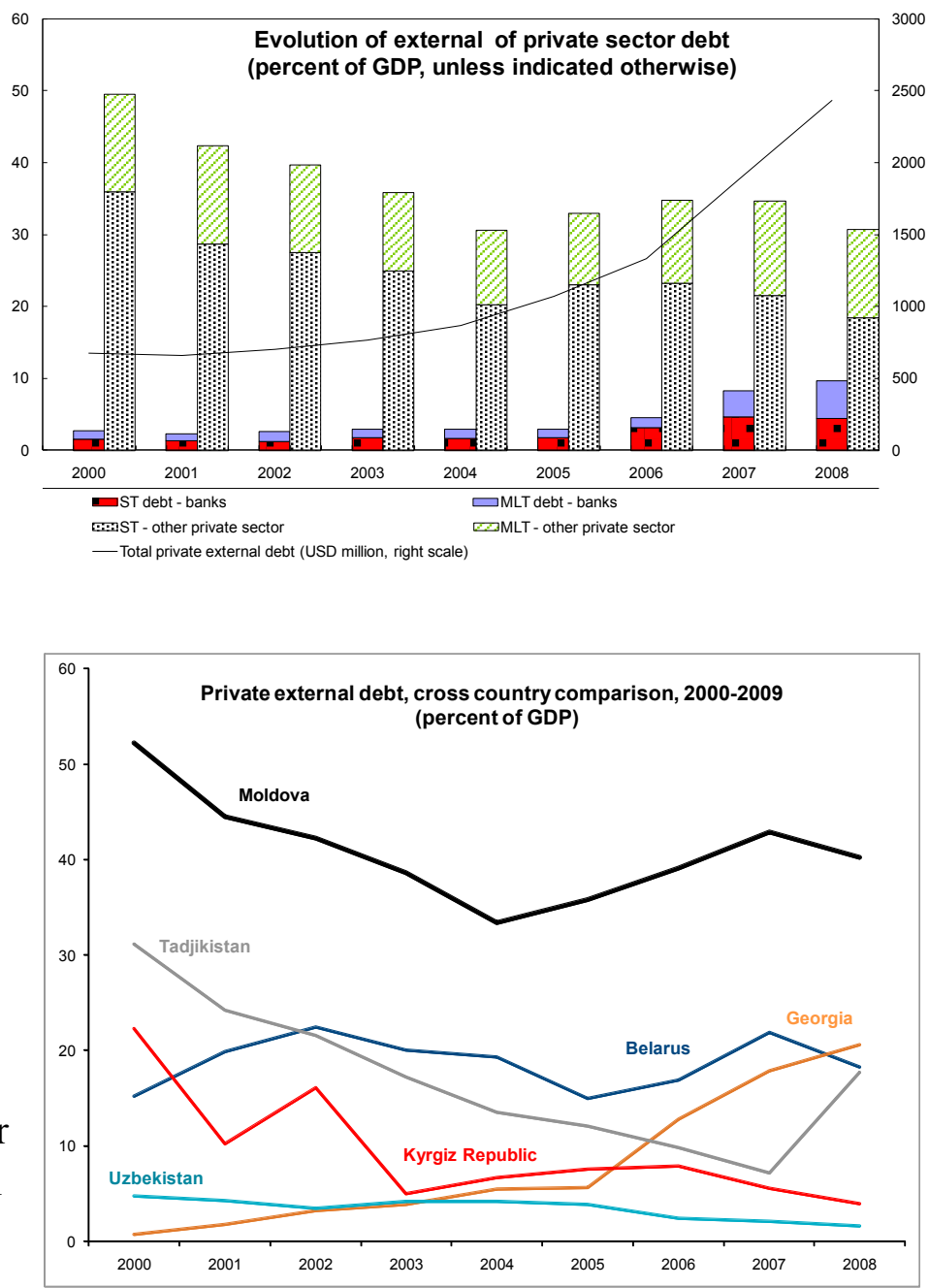
owned companies operating in Moldova. The share of private debt is very high by international standards, significantly exceeding the levels observed in other LICs and developing economies.

\section{MACROECONOMic FrameWork}

\section{The international financial crisis has worsened Moldova's macroeconomic} outlook significantly since the 2008 DSA exercise. In 2009, FDI came to a halt, domestic investment contracted sharply, and recession in the trading partners caused a severe decline in exports and remittances, contributing to a collapse in domestic demand. As a result, the economy went into a recession and external and fiscal financing gaps emerged. The baseline macroeconomic projections in this DSA take into account the expected sizeable fiscal and external adjustment, supported by significant borrowing in the near future in the context of the IMF arrangement (Box 1). ${ }^{12}$ Higher borrowing needed to fill the gaps will temporarily increase debt ratios, reversing recent gains in lowering the debt burden.

6. The macroeconomic assumptions differ with respect to the previous DSA due to the impact of recent global crisis. Key changes include a significantly lower GDP growth in the medium and long term on account of effects of the 2009 crisis, as well as a lower inflation rate. Recent reforms introduced by the government are projected to facilitate exports of agricultural products and exports of services, resulting in higher share of exports to GDP in the long-run than envisaged in the previous DSA, and a smaller current account deficit.

\section{EXTERnAl DebT SustainabiLity Under THE BASEline AND Alternative SCENARIOS}

\section{All external debt ratios remain well below the thresholds under the baseline} scenario, but the PV of the debt-to-GDP ratio temporarily breaches the threshold under two stress scenarios (Figures 1A-2A, Tables 1A-4A). ${ }^{13}$ Large borrowing in the next three years needed to fill the external and fiscal financing gaps will result in a temporary but significant increase in the level of external PPG debt to 35 percent of GDP in 2012. Due to the initial low levels of debt, the PV of the debt will not breach the threshold under the baseline scenario but could exceed it under two stress scenarios (lower non-debt creating

\footnotetext{
${ }^{12}$ The authorities requested a three-year blend of the Extended Credit Facility and the Extended Fund Facility with an access of SDR 369.6 million (300 percent of quota, or US\$588 million), split equally between the two arrangements.

${ }^{13}$ Average Country Policy and Institutional Assessment (CPIA) score for the last three years (2006-08) rates Moldova as a medium performer, and the DSA uses the indicative threshold indicators for countries in this category: 40 percent for the PV of debt-to-GDP ratio, 150 percent for the PV of debt-to-exports ratio, 250 percent for the PV of debt-to-revenue ratio, 20 percent for the debt service-to-exports ratio, and 30 percent for the debt service-to-revenue ratio. At the same time, in 2008 - the last year for which the CPIA score is available - the CPIA rated Moldova as a high performing country.
} 
flows and a decline in the growth of exports). ${ }^{14}$ These extreme scenarios, however, assume that exports do not pick up before 2012 after the 2009 collapse and remittances decline further from the very depressed 2009 levels. Taking into account the ongoing recovery in Moldova's trading partners, as well as the dismantling of trade restrictions by the new government, exports are not likely to remain stagnant. Remittances, which before the 2009 crisis were one of the highest in the world as percentage of GDP, are also unlikely to decrease any further, mitigating the risks.

8. While rebound in exports, fiscal consolidation and significant levels of remittances should ensure that adequate resources for public debt service remain available, certain risks exist. While none of the indicators of debt service breaches the threshold, some liquidity pressures could emerge in the medium term when the repurchases of the ECF and the EFF are falling due as shown by an increase in ratios of debt service to exports and revenues under the extreme scenarios. In addition, significant private external debt implies that private borrowers would compete with the public sector for foreign exchange needed to service their external debt.

\section{Public Debt Sustainability under the Baseline And Alternative Scenarios}

9. Under the baseline, the ratios of total public debt do not signal increased vulnerability at present. Total public debt consists mainly of external PPG debt (72 percent) hence it follows closely the dynamics of its external component. Under the baseline scenario, after the initial increase over the medium-term, the PV of total public debt-to-GDP ratio will decline to 24 percent, while the PV of debt service to revenue ratio would fluctuate around 7 percent.

10. The most significant increase of public debt indicators would materialize if the primary balance remains at the unsustainable 2009 level. Large and persistent primary gap would then lead to an explosive debt dynamics. However, the ongoing process of fiscal consolidation, combined with binding financing constraints should result in a structural reversal of the fiscal position, rendering such scenario less probable.

11. Two stress scenarios could result in a sharp increase of public debt indicators. Permanently lower GDP growth results in an ever-increasing PV of debt-to-GDP ratio reaching 87 percent in 2029, almost three times larger compared to the current level. A sharp real exchange rate depreciation could result in a spike in public sector debt but this increase would be temporary, and debt dynamics would revert to a benign pattern in the medium term.

\footnotetext{
${ }^{14}$ In Moldova, remittances are classified as either current transfers or compensation of employees. Both categories are included in the DSA (under "current transfers").
} 


\section{Box 1. Macroeconomic Assumptions behind the DSA}

During the projection period (2009-2029), real GDP is projected to grow by 3.4 percent on average. After the initial decline of 9 percent in 2009, growth will gradually rise to an average of 2.3 percent in 2010-2011 and to 5 percent in 2012-2014, led by a rebound in exports, investment and remittances. In the long-run, the negative output gap will close and growth will decline to 4 percent.

Inflation is projected to remain in single digits, increasing somewhat in 2010 as the economy recovers, but subsiding to 4 percent in the long-run (as measured by the GDP deflator). This projection assumes sound public sector policies and strong commitment of the National Bank to preserving price stability.

Exports are expected to be an important driver of growth. They are projected to accelerate in the medium term, as the trading partners recover from the recent crisis, internal restrictions on exports of wine and other agricultural products are removed, and Moldova makes full use of the autonomous trade preferences agreement with the EU. In addition, structural reforms aimed at improving the business environment, demonopolizing telecommunication services and expanding access to broadband internet should result in strong growth of services, in particular software development. As a result, exports of goods and services are projected to reach 59 percent of GDP in 2029.

Imports are projected to expand as well, fuelled by intermediate imports needed for the exports sector, as well as driven by the rebound in investment and private consumption. In the long-term, however, growth of imports will subside somewhat as domestic production of tradables expands.

Remittances are projected to gradually recover from the depressed 2009 level in the mediumterm. In the long-term, however, as the economy develops, more employment options are available domestically, and migrants abroad lose ties with the home country, remittances are projected to decline relative to GDP from 32 percent to 29 percent of GDP.

The current account deficit is projected to widen in the short run, and to stabilize around 89 percent of GDP in the medium and long term. It will be financed by FDI that is expected to recover to the pre-boom levels of 7.2 percent of GDP in the long run, supported by structural reforms aimed at improving the business climate.

The primary fiscal deficit is projected to decline in the medium term as a result of fiscal consolidation undertaken by the authorities. In the long run, revenues are projected to increase by 3.2 percent of GDP, while rationalization of primary noninterest expenditures will cause their decline by 4.4 percent of GDP, resulting in a primary surplus of 0.1 percent of GDP.

Borrowing assumptions reflect the gradual shift of Moldova away from concessional financing. Concessional loans are projected to decline from 88 percent of total borrowing in 2015 to 23 percent in 2029. At the same time, borrowing from commercial sources is projected to increase, reaching 55 percent of the total in 2029. 


\section{SCENARIOS WITH ADdITIONAL NON-CONCESSIONAL BORROWING}

12. This section explores alternative scenarios where the government increases its non-concessional borrowing by additional US\$125 million a year over ten years. ${ }^{15}$ The non-concessional resources could be invested in infrastructure upgrades (e.g., roads, energy supply and distribution, water treatment, and agricultural irrigation). It is assumed that the loans will be repaid within 15 years, with 3 year grace period, and will carry an interest rate of 3 percent per annum.

\section{Scenario 1}

13. The first scenario envisages that the additional borrowing will contribute to a higher real growth rate of GDP in the medium and long term than under the baseline scenario. In the absence of specific information on the projects to be financed with new loans, it is conservatively assumed that their "domestic component" will be about 30 percent, while the remaining amount will directly translate into increased imports. Given large spare capacities in the economy after the crisis, the projects' implementation can directly contribute to the GDP growth without rekindling inflation pressures. Therefore in the medium term, annual growth could rise by $0.5-0.6$ percent under a conservative scenario, mainly as a result of higher investment, employment, and consumption during project implementation years. Additional infrastructure investment will also raise the economy's capital stock and boost productivity, ensuring higher growth in the long run (4.5 percent, or 0.5 percentage points higher than under the baseline).

14. Exports and FDI will initially rise only in line with GDP, but then will accelerate in the long run, compared with the baseline scenario. Modest initial growth will reflect lags in improvements in infrastructure. In the long run, better infrastructure will improve Moldova's attractiveness to foreign investors, raising FDI to 9 percent of GDP and exports of goods to 43 percent of GDP.

15. Imports will accelerate substantially over the course of the operation, causing initial deterioration of the current account (Figures $3 \mathrm{~A}-\mathbf{4 A}$, Tables $5 \mathrm{~A}-\mathbf{8 A}$ ). In the long run, however, Moldova's reliance on imported energy will decrease due to new investment, contributing to a stabilization of imports at 79 percent relative to GDP. The current account in the long term will remain broadly unchanged from the baseline scenario (Section III).

\footnotetext{
${ }^{15}$ The amount is in line with the ceiling on nonconcessional external borrowing set in the Fund-supported program for 2010. This ceiling excludes borrowing from international financial institutions (IFIs). Availability of external financing, changes in the macroeconomic framework and the debt sustainability outlook, and the new Fund policy on debt limits will be taken into account in discussions of the quantitative targets on debt accumulation in subsequent years during program reviews.
} 
16. Under these assumptions, the external debt burden indicators generally remain under the indicative thresholds, although the PV of the debt-to-GDP ratio could breach its threshold under some stress tests, signaling increased vulnerabilities. Two tests, however, employ the unlikely assumptions that remittances would experience a further sharp drop in 2010-11 from their already depressed 2009 level. Two other tests are more realistic, assuming respectively that exports will take a long time to recover from the 2009 slump and a sharp real depreciation (30 percent), above levels observed in Moldova during the recent crisis, but comparable to the shocks in the late 1990s. The PV of the debt-to-GDP ratio also breaches the threshold if the financing terms of the new loans are much less favorable than assumed here. The probability of this scenario will strongly depend on the financing terms of the new non-concessional financing. If the authorities succeed in obtaining financing at fixed interest rate, the risk is less likely to materialize. On the other hand, floating interest rates linked to the international interest rates would significantly increase Moldova's debt vulnerability. Overall, this scenario emphasizes the need to contract additional financing needed for development projects on fixed (preferably concessional) terms.

\section{Scenario 2}

17. The second (conservative) scenario assumes a similar schedule of borrowing as in Scenario 1, but no additional increase in growth and exports (Figure 5A-6A, Tables 9A-12A). Additional borrowing will thus only result in increased imports, a wider current account deficit, and higher debt burden in the long run. These conservative assumptions are motivated by instances of international experience with large infrastructure projects, where actual benefits turn out much lower than expected.

18. Under Scenario 2, the baseline external debt burden indicators remain under the thresholds, while the PV of debt-to-GDP ratio exceeds the threshold under some of the extreme tests. The outcome is similar to Scenario 1, indicating that the relatively benign picture in that scenario does not depend on assumptions about rising growth and exports.

\section{DebT Distress Classification ANd ConClusions}

19. The risk of public debt distress in Moldova is low at present, but alternative scenarios indicate certain vulnerabilities. Under the baseline scenario, all indicators of PPG external debt remain in safe territory, except under the most extreme stress tests. Significant borrowing under the new Fund arrangement, combined with financing from other international partners, will increase the debt burden, but only temporarily, and to manageable levels. Stress tests under alternative scenarios, however, point to a potentially significant risk stemming from the interaction of stepped-up non-concessional borrowing with sluggish export growth and interest and exchange rate shocks, highlighting the need to seek financing at concessional terms, or at least at a fixed and low interest rate. 
20. Moreover, the sizeable private external debt, the arrears on energy payments and Moldova's history of debt distress warrant additional caution in public borrowing. Private debt is significantly larger than PPG debt and exceeds by far levels observed in other LICs. It could generate balance of payments pressures by competing with public debt for foreign exchange needed to cover the debt service. Moreover, the existing payment arrears for energy resources are a potential source of fiscal liability, though these risks are mitigated by the authorities' plans to ensure tariff setting at cost-recovery levels. Moldova's previous debt difficulties - the Eurobond rescheduling in 2002 and the Paris Club rescheduling in 2006 - also calls for careful evaluation of the terms and uses of prospective external public borrowing. 
Figure 1A. Moldova: Indicators of Public and Publicly Guaranteed External Debt under Alternative Scenarios, 2009-2029 1/
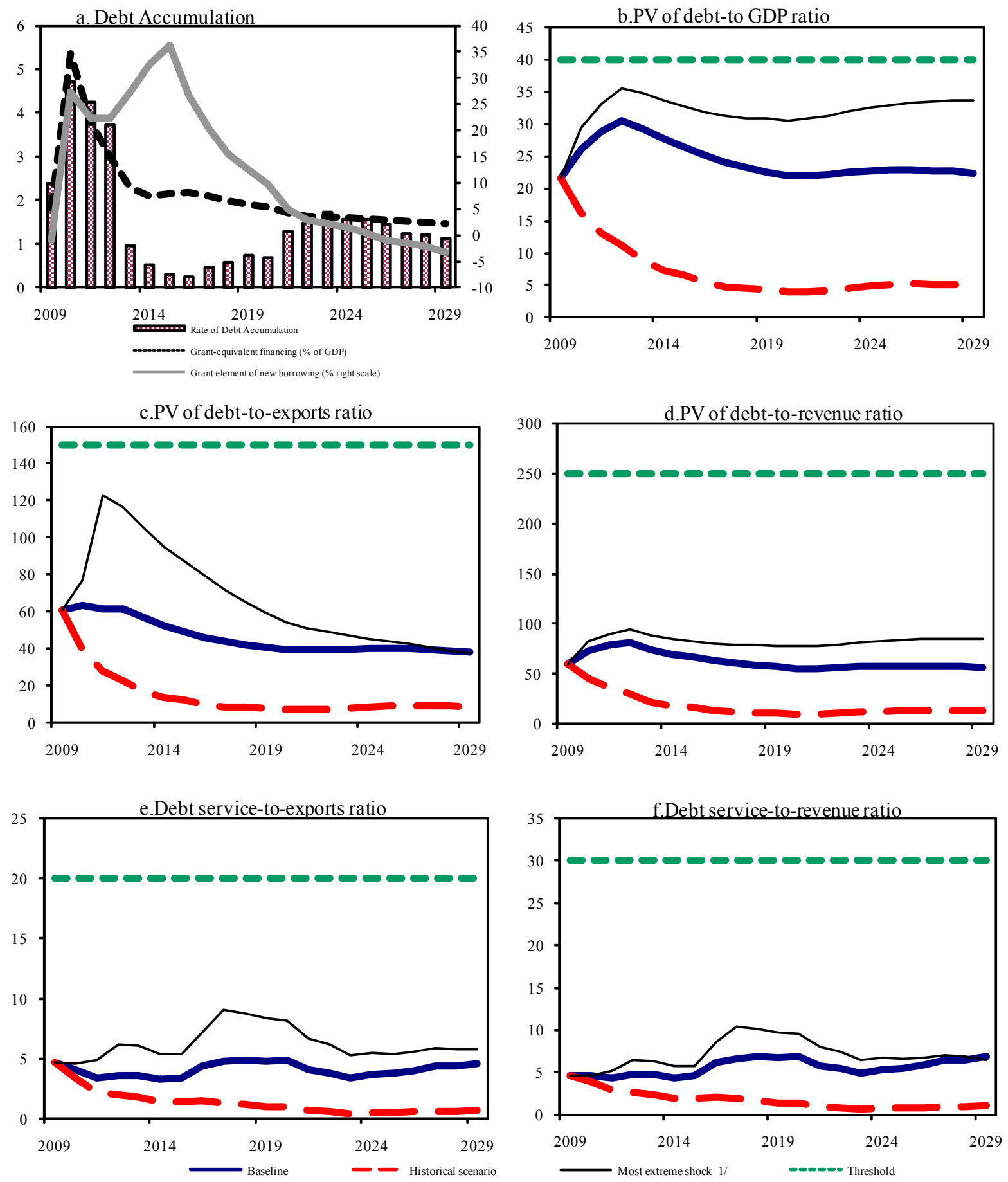

Sources: Country authorities; and staff estimates and projections.

1/ The most extreme stress test is the test that yields the highest ratio in 2019. In figure b. it corresponds to a Terms shock; in c. to a Exports shock; in d. to a Terms shock; in e. to a Exports shock and in figure f. to a Non-debt flows shock 
Figure 2A.Moldova: Indicators of Public Debt Under Alternative Scenarios, 2009-2029 1/

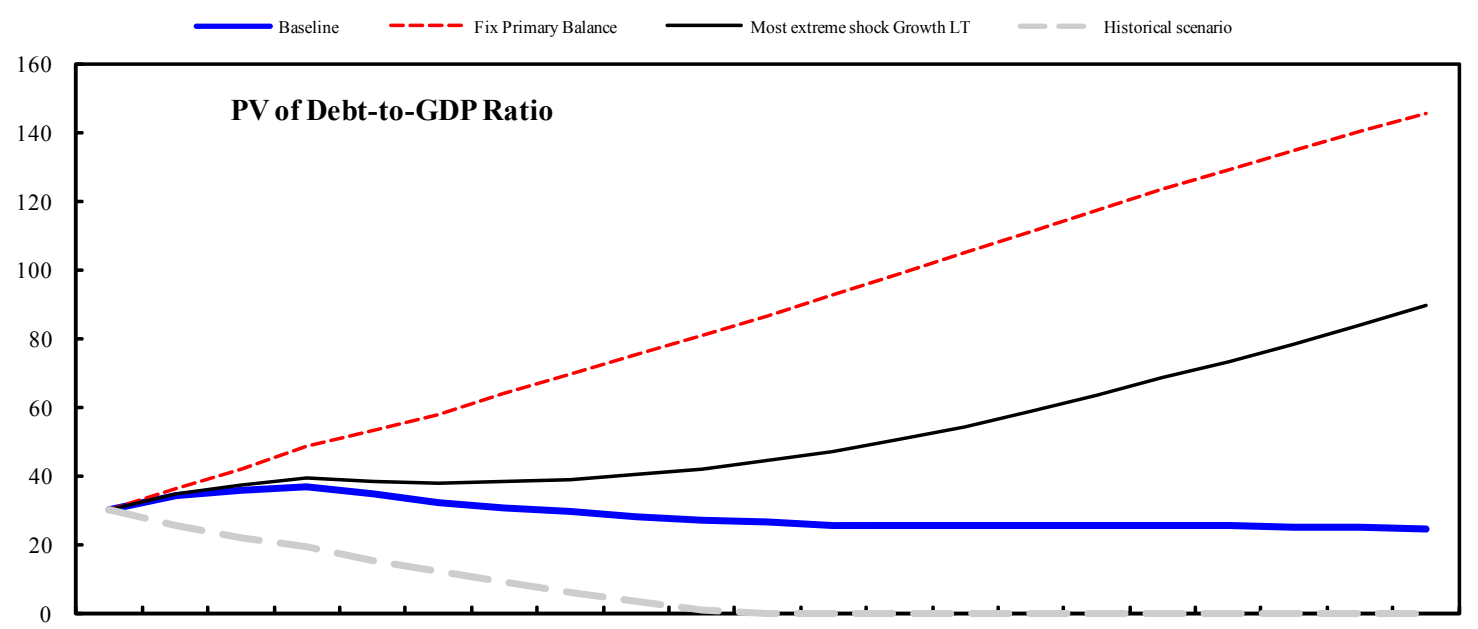

$20092010201120122013201420152016201720182019202020212022 \quad 2023 \quad 202420252026202720282029$

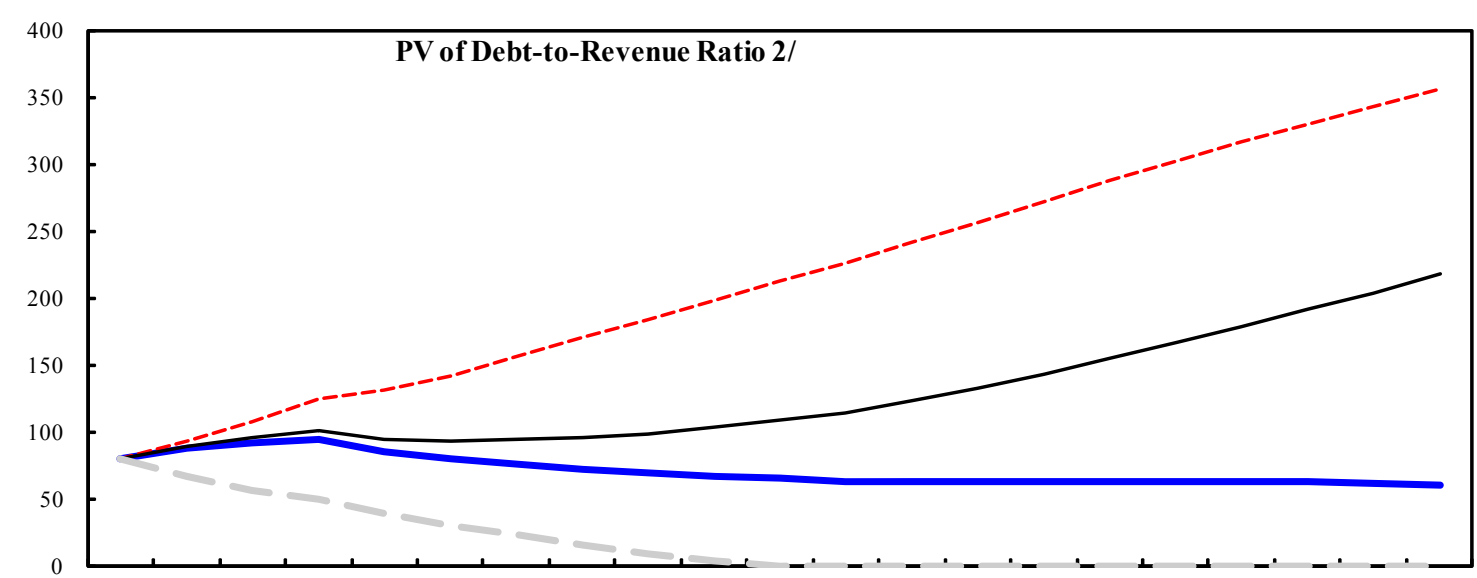

$200920102011201220132014201520162017 \quad 20182019202020212022 \quad 2023202420252026202720282029$

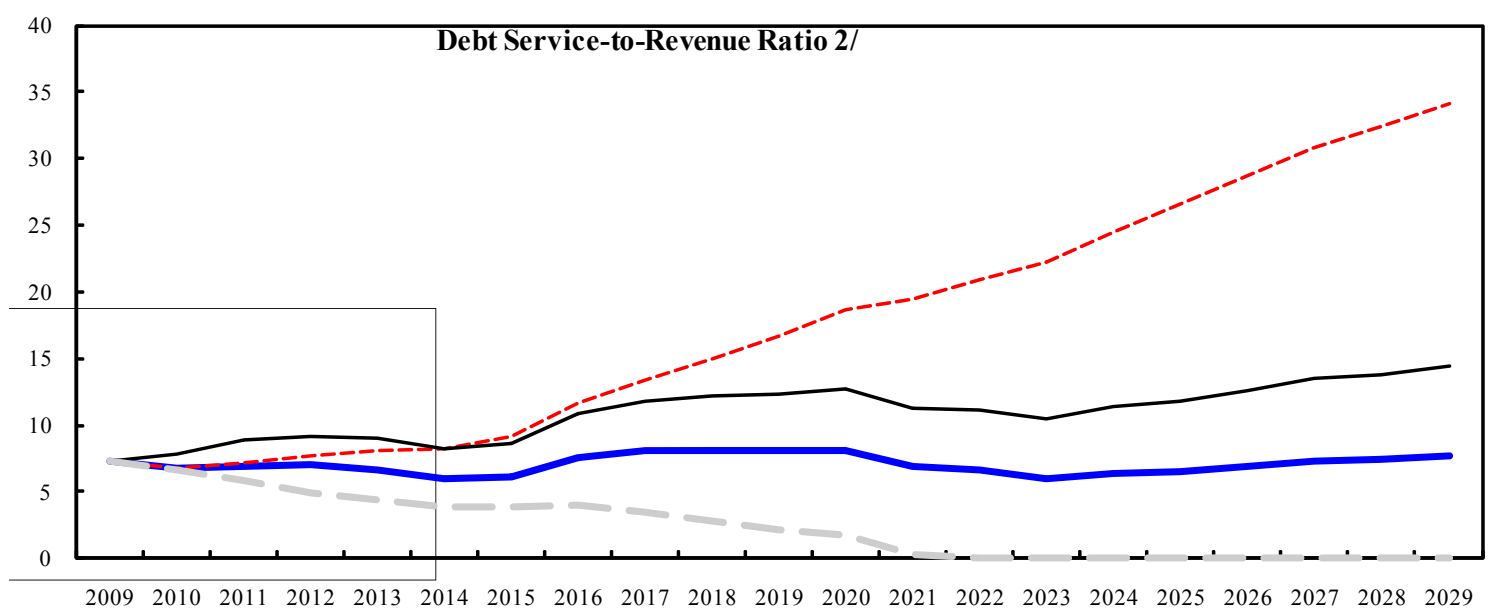

Sources: Country authorities; and staff estimates and projections.

$1 /$ The most extreme stress test is the test that yields the highest ratio in 2019.

2/ Revenues are defined inclusive of grants. 
Figure 3A. Moldova: Indicators of Public and Publicly Guaranteed External Debt with Additional Non-Concessional Borrowing, and Positive Impact on Growth, 2009-2029 1/
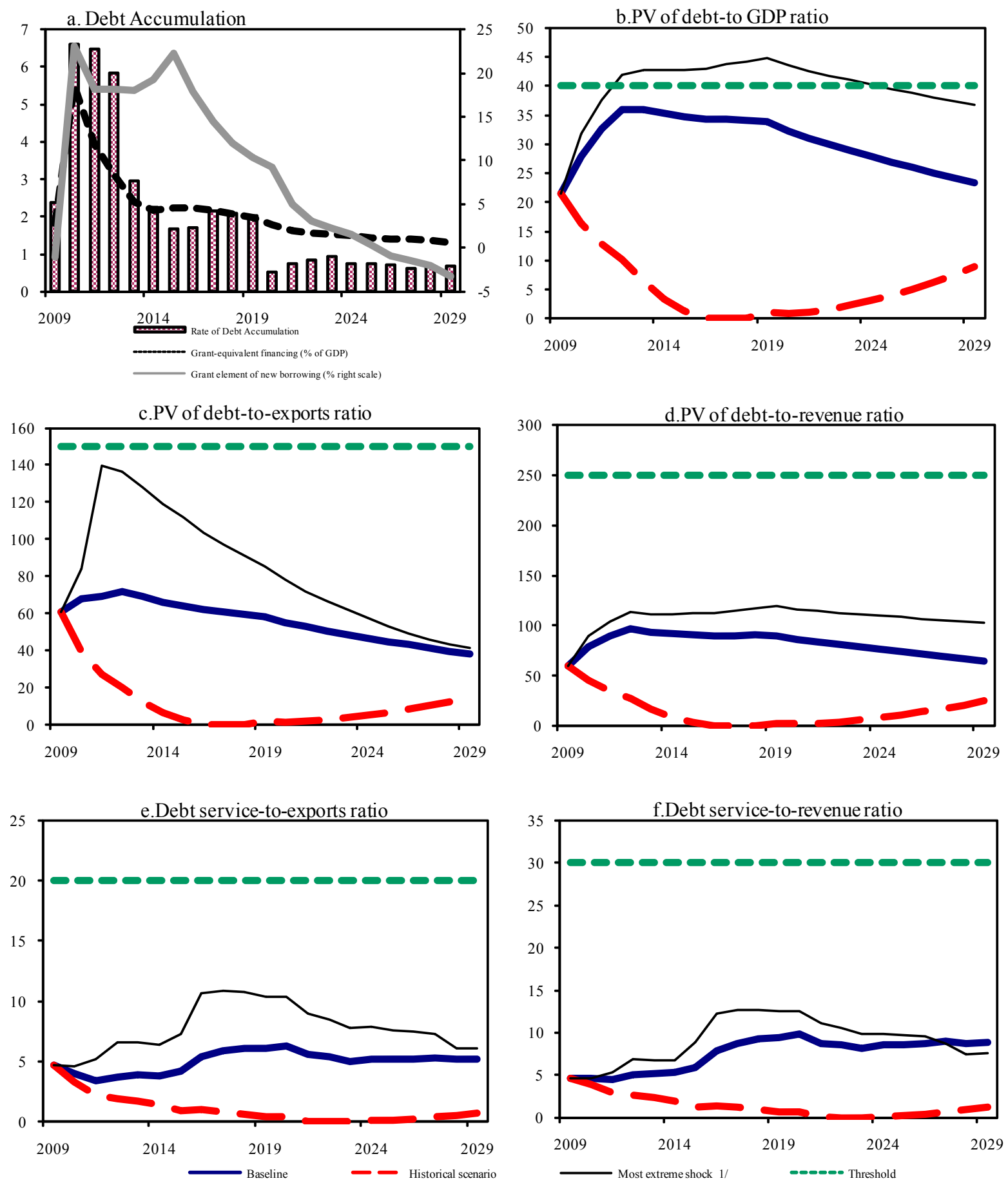

Sources: Country authorities; and staff estimates and projections.

1/ The most extreme stress test is the test that yields the highest ratio in 2019. In figure b. it corresponds to a Terms shock; in c. to a Exports shock; in d. to a Terms shock; in e. to a Exports shock and in figure f. to a Non-debt flows shock 
Figure 4A.Moldova: Indicators of Public Debt with Additional Non-Concessional Borrowing, and Positive Impact on Growth, 2009-2029 1/

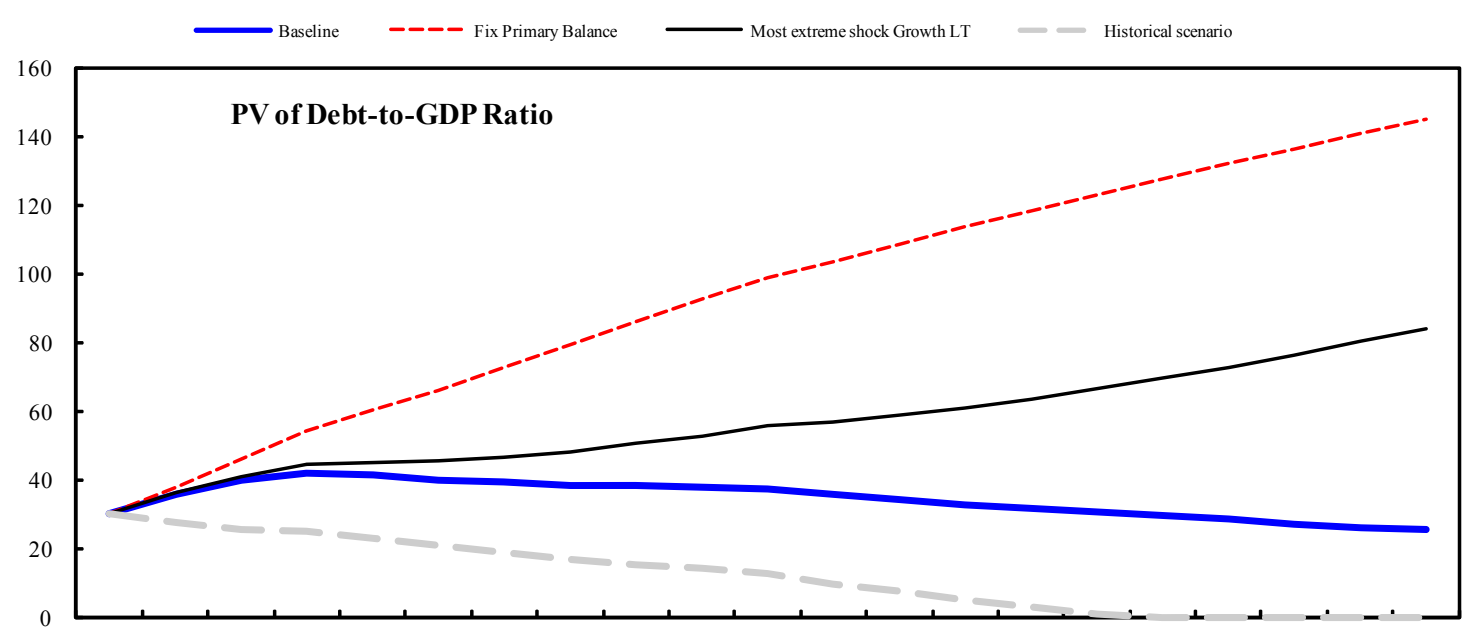

$20092010201120122013201420152016201720182019202020212022 \quad 2023202420252026202720282029$

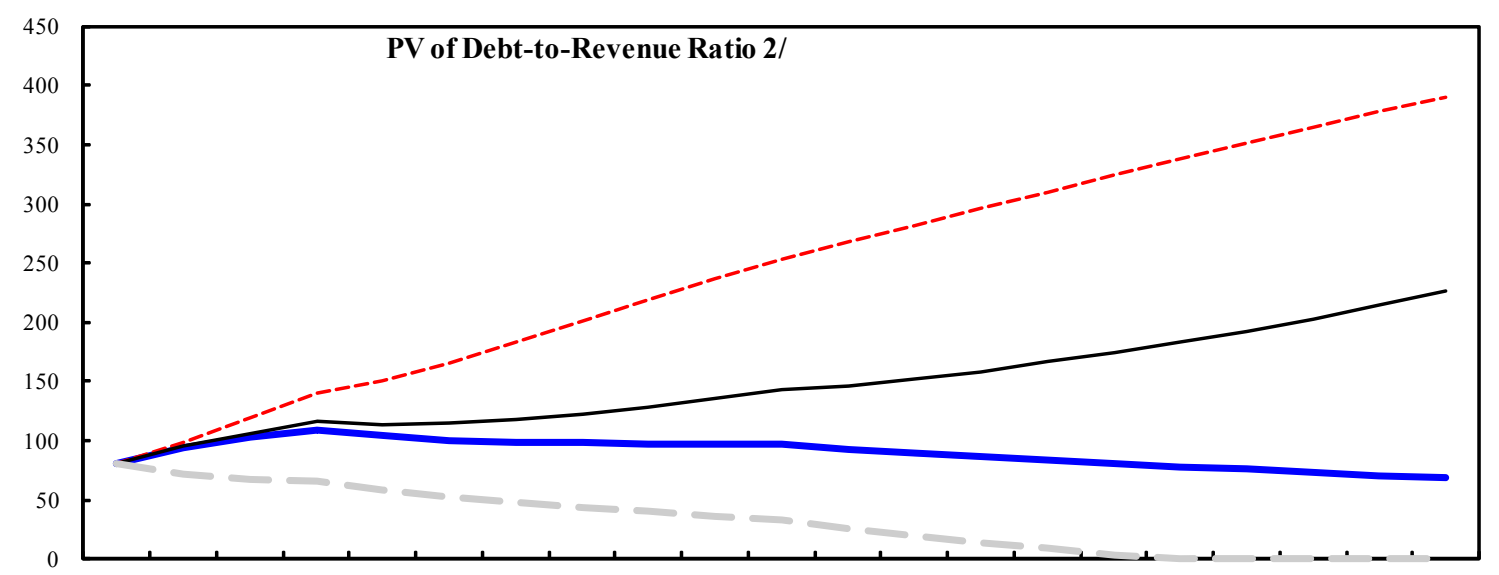

$200920102011201220132014201520162017 \quad 20182019202020212022 \quad 2023202420252026202720282029$

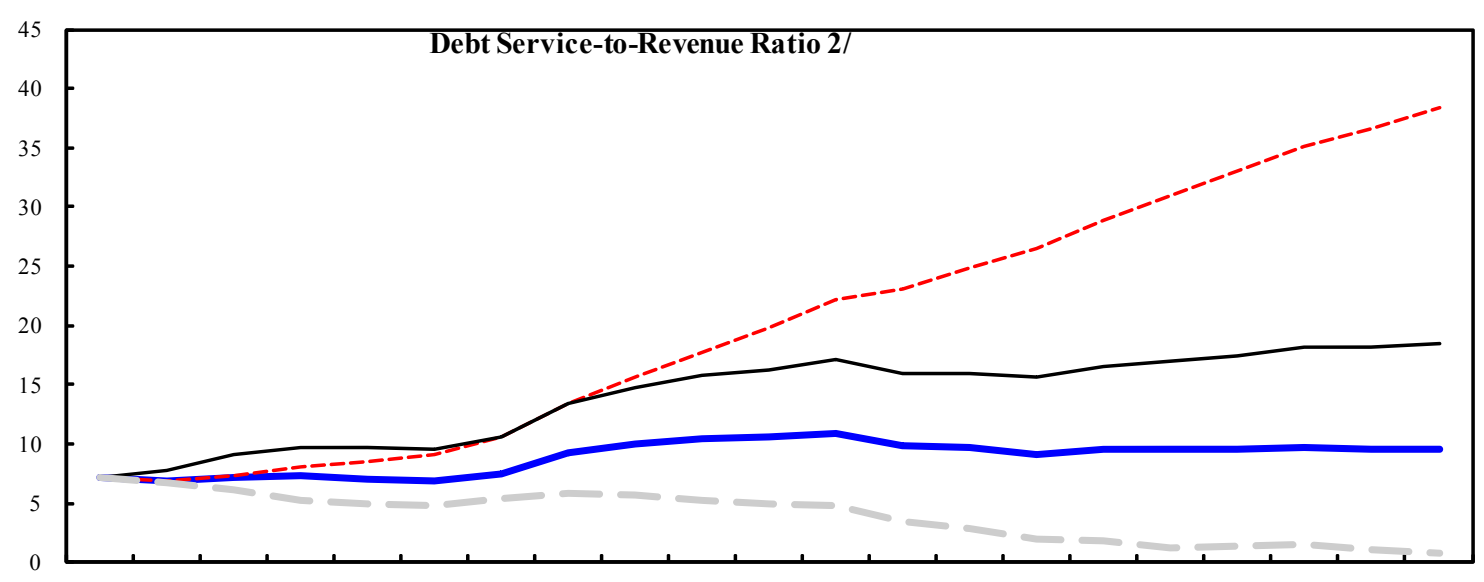

$2009201020112012 \quad 2013201420152016 \quad 2017 \quad 2018 \quad 20192020 \quad 20212022 \quad 2023 \quad 202420252026202720282029$

Sources: Country authorities; and staff estimates and projections.

1/ The most extreme stress test is the test that yields the highest ratio in 2019.

2/ Revenues are defined inclusive of grants. 
Figure 5A. Moldova: Indicators of Public and Publicly Guaranteed External Debt with Additional Non-Concessional Financing and no Increase in Growth, 2009-2029 1/
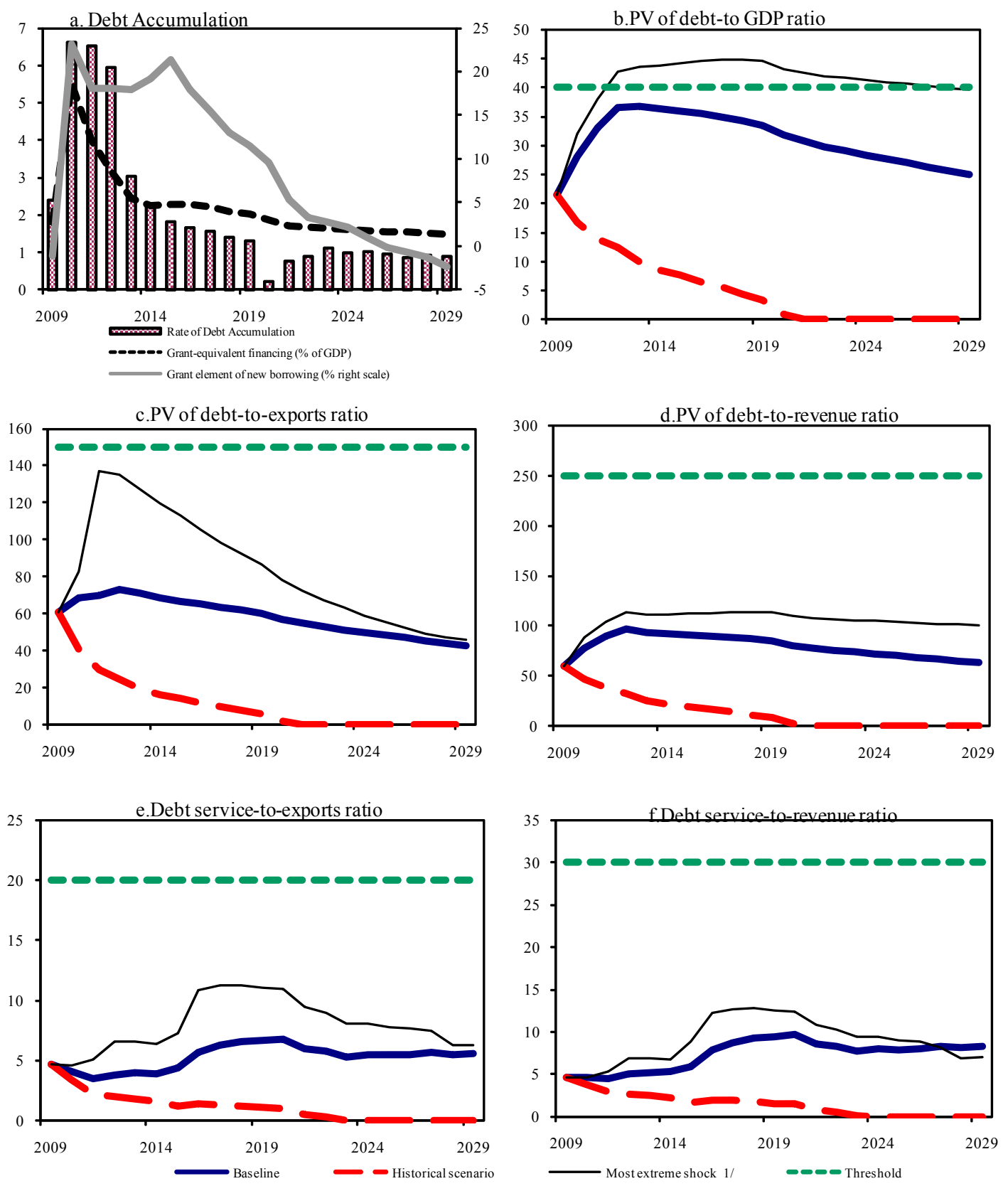

Sources: Country authorities; and staff estimates and projections.

1/ The most extreme stress test is the test that yields the highest ratio in 2019. In figure b. it corresponds to a Terms shock; in c. to a Exports shock; in d. to a Terms shock; in e. to a Exports shock and in figure f. to a Non-debt flows shock 
Figure 6A.Moldova: Indicators of Public Debt with Additional Non-Concessional Financing and no Increase in Growth, 2009-2029 1/

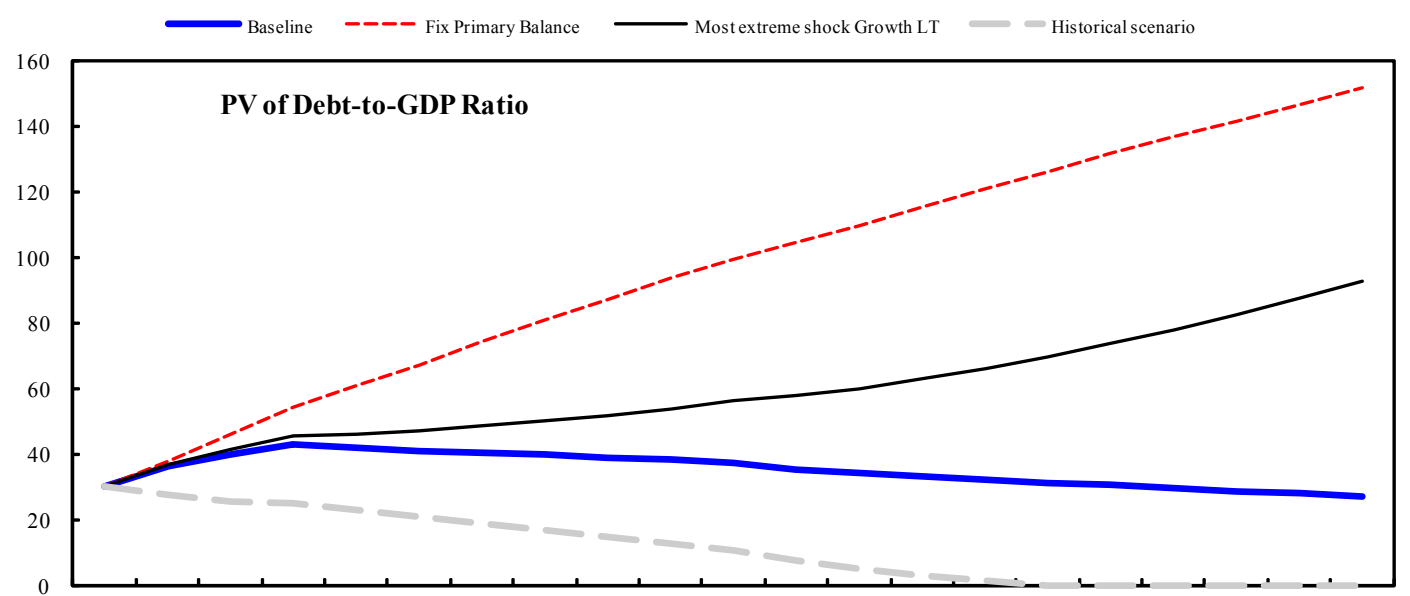

$20092010201120122013201420152016201720182019202020212022 \quad 20232024 \quad 20252026202720282029$

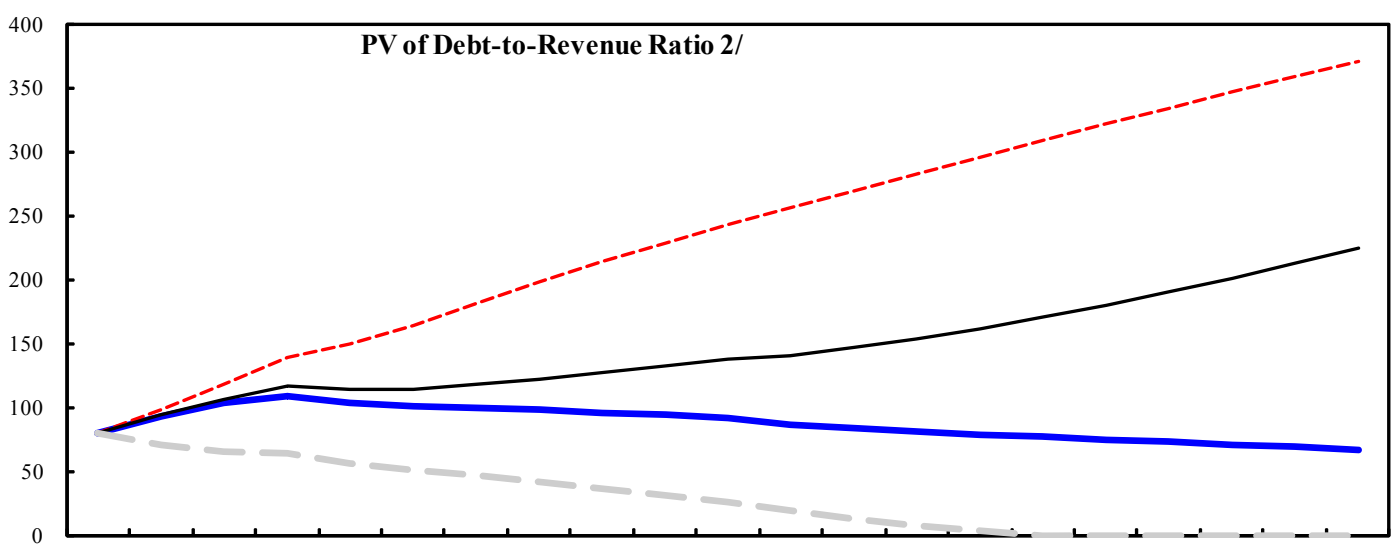

$2009201020112012201320142015201620172018 \quad 20192020 \quad 20212022 \quad 2023202420252026202720282029$

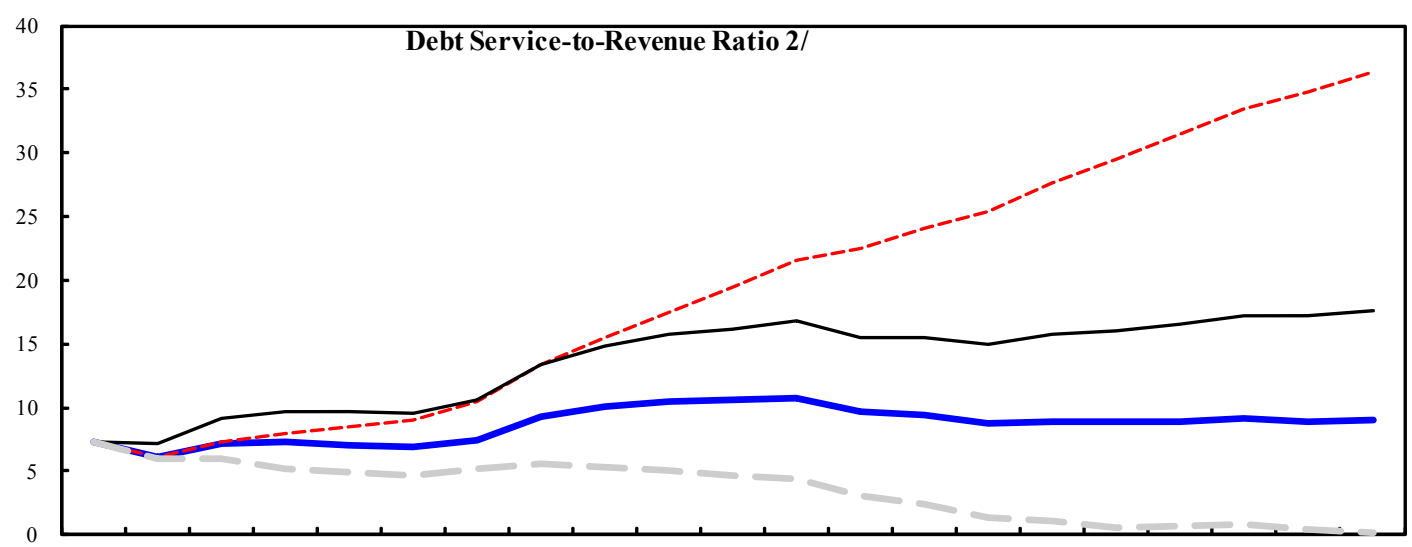

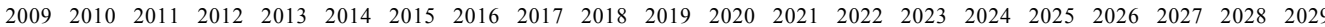

Sources: Country authorities; and staff estimates and projections.

$1 /$ The most extreme stress test is the test that yields the highest ratio in 2019.

$2 /$ Revenues are defined inclusive of grants. 
Table 1A. Moldova: External Debt Sustainability Framework, Baseline Scenario, 2006-2029 1/

\begin{tabular}{|c|c|c|c|c|c|c|c|c|c|c|c|c|c|c|c|}
\hline & \multicolumn{3}{|c|}{ Actual } & \multirow{3}{*}{$\begin{array}{l}\text { Historical } \\
\text { Average }\end{array}$} & \multirow{3}{*}{$\begin{array}{c}\text { Standard } \\
\text { Deviation }\end{array}$} & \multicolumn{5}{|c|}{ Projections } & \multirow{2}{*}{\multicolumn{2}{|c|}{$2009-2014$}} & \multirow[b]{3}{*}{2019} & \multirow[b]{3}{*}{2029} & \multirow{3}{*}{$\begin{array}{c}2015-2029 \\
\text { Average }\end{array}$} \\
\hline & & & & & & & & & & & & & & & \\
\hline & 2006 & 2007 & 2008 & & & 2009 & 2010 & 2011 & 2012 & 2013 & 2014 & Average & & & \\
\hline External debt (nominal) $1 /$ & 64.7 & 62.7 & 55.9 & & & 68.1 & 79.3 & 84.2 & 86.4 & 84.8 & 82.7 & & 76.7 & 67.6 & \\
\hline $\mathrm{o} / \mathrm{w}$ public and publicly guaranteed (PPG) & 25.6 & 19.8 & 15.7 & & & 22.4 & 28.7 & 32.3 & 34.6 & 33.1 & 31.3 & & 25.7 & 23.1 & \\
\hline Change in external debt & 2.5 & -2.0 & -6.8 & & & 12.2 & 11.2 & 4.9 & 2.2 & -1.6 & -2.1 & & -1.2 & -1.0 & \\
\hline Identified net debt-creating flows & -2.1 & -9.3 & -10.7 & & & 13.9 & 6.8 & 5.4 & 2.3 & 0.1 & -0.9 & & -0.2 & -0.4 & \\
\hline Non-interest current account deficit & 10.3 & 15.5 & 16.6 & 6.0 & 6.6 & 7.5 & 7.9 & 8.7 & 7.3 & 6.2 & 5.3 & & 5.5 & 5.4 & 5.5 \\
\hline Deficit in balance of goods and services & 47.1 & 52.5 & 53.0 & & & 33.0 & 37.3 & 38.2 & 37.6 & 37.3 & 37.0 & & 36.0 & 34.0 & \\
\hline Exports & 44.8 & 45.4 & 41.0 & & & 35.6 & 40.8 & 47.0 & 49.6 & 51.5 & 52.8 & & 55.4 & 58.2 & \\
\hline Imports & 91.9 & 97.9 & 94.0 & & & 68.5 & 78.1 & 85.3 & 87.2 & 88.7 & 89.8 & & 91.4 & 92.3 & \\
\hline Net current transfers (negative $=$ inflow) & -39.1 & -40.0 & -38.4 & -29.9 & 8.5 & -27.2 & -32.4 & -32.5 & -33.1 & -33.9 & -34.5 & & -32.6 & -29.2 & -31.6 \\
\hline o/w official & -1.9 & -1.7 & -2.0 & & & -1.5 & -3.2 & -2.5 & -1.8 & -1.7 & -1.6 & & -1.6 & -1.6 & -37.0 \\
\hline Other current account flows (negative $=$ net inflow) & 2.3 & 3.0 & 1.9 & & & 1.7 & 3.1 & 2.9 & 2.9 & 2.9 & 2.8 & & 2.1 & 0.6 & \\
\hline Net FDI (negative $=$ inflow) & -6.9 & -11.9 & -11.4 & -6.5 & 3.4 & -1.7 & -3.8 & -4.8 & -5.2 & -5.9 & -6.5 & & -6.8 & -7.2 & -6.9 \\
\hline Endogenous debt dynamics 2 / & -5.5 & -13.0 & -15.9 & & & 8.1 & 2.7 & 1.6 & 0.1 & -0.3 & 0.3 & & 1.1 & 1.4 & \\
\hline Contribution from nominal interest rate & 2.1 & 1.6 & 1.3 & & & 2.5 & 3.8 & 3.9 & 4.1 & 3.8 & 4.2 & & 4.0 & 3.9 & \\
\hline Contribution from real GDP growth & -2.6 & -1.5 & -3.6 & & & 5.7 & -1.1 & -2.3 & -3.9 & -4.0 & -3.9 & & -2.9 & -2.6 & \\
\hline Contribution from price and exchange rate changes & -5.1 & -13.1 & -13.6 & & & & & & & & & & & & \\
\hline Residual (3-4) 3/ & 4.7 & 7.3 & 3.9 & & & -1.7 & 4.4 & -0.5 & -0.1 & -1.7 & -1.2 & & -1.0 & -0.6 & \\
\hline $\mathrm{o} / \mathrm{w}$ exceptional financing & -2.5 & -3.1 & -2.6 & & & -1.3 & 0.0 & 0.0 & 0.0 & 0.0 & 0.0 & & 0.0 & 0.0 & \\
\hline PV of external debt $4 /$ & $\ldots$ & $\ldots$ & 55.1 & & & 67.4 & 76.6 & 80.7 & 82.3 & 80.9 & 79.1 & & 73.5 & 66.9 & \\
\hline In percent of exports & $\ldots$ & $\ldots$ & 134.3 & & & 189.3 & 187.7 & 171.7 & 165.9 & 157.2 & 149.6 & & 132.8 & 114.9 & \\
\hline PV of PPG external debt & $\ldots$ & $\ldots$ & 14.9 & & & 21.6 & 26.0 & 28.8 & 30.6 & 29.2 & 27.6 & & 22.6 & 22.4 & \\
\hline In percent of exports & $\ldots$ & $\ldots$ & 36.3 & & & 60.7 & 63.7 & 61.3 & 61.6 & 56.8 & 52.3 & & 40.8 & 38.5 & \\
\hline In percent of government revenues & $\ldots$ & $\ldots$ & 38.3 & & & 60.2 & 73.0 & $\mathbf{7 8 . 5}$ & 81.6 & 74.7 & 70.1 & & 57.3 & 56.8 & \\
\hline Debt service-to-exports ratio (in percent) & 15.1 & 13.7 & 16.6 & & & 19 & 21 & 17 & 18 & 17 & 19 & & 24.5 & 24.6 & \\
\hline PPG debt service-to-exports ratio (in percent) & 7.0 & 3.9 & 3.3 & & & 4.7 & 4.1 & 3.4 & 3.6 & 3.6 & 3.3 & & 4.8 & 4.6 & \\
\hline PPG debt service-to-revenue ratio (in percent) & 8.0 & 4.4 & 3.5 & & & 4.6 & 4.7 & 4.3 & 4.7 & 4.8 & 4.4 & & 6.8 & 6.8 & \\
\hline Total gross financing need (Millions of U.S. dollars) & 1118.9 & 1332.9 & 1865.3 & & & 2057.4 & 2003.2 & 1988.1 & 2007.1 & 1918.0 & 1957.8 & & 2446.1 & 3368.6 & \\
\hline Non-interest current account deficit that stabilizes debt ratio & 7.7 & 17.5 & 23.3 & & & -4.7 & -3.3 & 3.8 & 5.1 & 7.9 & 7.4 & & 6.7 & 6.4 & \\
\hline \multicolumn{16}{|l|}{ Key macroeconomic assumptions } \\
\hline Real GDP growth (in percent) & 4.8 & 3.0 & 7.8 & 5.0 & 3.6 & -9.0 & 1.5 & 3.0 & 5.0 & 5.0 & 5.0 & 1.8 & 4.0 & 4.0 & 4.0 \\
\hline GDP deflator in US dollar terms (change in percent) & 8.9 & 25.4 & 27.6 & 9.4 & 15.7 & -2.8 & -6.3 & 1.6 & 1.9 & 2.1 & 2.4 & -0.2 & 2.0 & 2.0 & 2.0 \\
\hline Effective interest rate (percent) $5 /$ & 3.9 & 3.2 & 2.8 & 4.1 & 0.8 & 3.9 & 5.3 & 5.1 & 5.2 & 4.7 & 5.3 & 4.9 & 5.5 & 6.1 & 5.8 \\
\hline Growth of exports of G\&S (US dollar terms, in percent) & 1.5 & 30.9 & 24.3 & 13.2 & 15.7 & -23.2 & 9.1 & 20.5 & 13.0 & 11.2 & 10.4 & 6.8 & 6.6 & 6.6 & 6.8 \\
\hline Growth of imports of G\&S (US dollar terms, in percent) & 15.3 & 37.5 & 32.1 & 18.7 & 20.9 & -35.5 & 8.4 & 14.2 & 9.5 & 9.2 & 8.9 & 2.4 & 6.3 & 6.2 & 6.3 \\
\hline Grant element of new public sector borrowing (in percent) & & & & & $\ldots$ & -1.1 & 27.3 & 22.2 & 22.4 & 27.3 & 32.5 & 21.8 & 12.4 & -3.3 & 8.3 \\
\hline Government revenues (excluding grants, in percent of GDP) & 39.2 & 39.9 & 38.9 & & & 35.9 & 35.6 & 36.7 & 37.5 & 39.1 & 39.4 & & 39.4 & 39.4 & 39.4 \\
\hline Aid flows (in Millions of US dollars) $7 /$ & 46.6 & 126.1 & 127.0 & & & 123.4 & 249.4 & 190.9 & 162.8 & 162.8 & 162.8 & & 188.0 & 254.0 & \\
\hline $\mathrm{o} / \mathrm{w}$ Grants & 24.0 & 79.9 & 102.8 & & & 99.7 & 161.4 & 130.9 & 102.8 & 102.8 & 102.8 & & 138.0 & 249.0 & \\
\hline $\mathrm{o} / \mathrm{w}$ Concessional loans & 22.6 & 46.2 & 24.2 & & & 23.7 & 88.0 & 60.0 & 60.0 & 60.0 & 60.0 & & 50.0 & 5.0 & \\
\hline Grant-equivalent financing (in percent of GDP) $8 /$ & $\ldots$ & $\ldots$ & $\ldots$ & & & 1.8 & 5.4 & 3.8 & 3.0 & 2.3 & 2.1 & & 1.9 & 1.5 & 1.8 \\
\hline Grant-equivalent financing (in percent of external financing) $8 /$ & $\ldots$ & $\ldots$ & $\ldots$ & & & 33.1 & 47.9 & 44.8 & 41.8 & 58.2 & 65.3 & & 43.3 & 33.6 & 42.0 \\
\hline \multicolumn{16}{|l|}{ Memorandum items: } \\
\hline Nominal GDP (Millions of US dollars) & 3408.4 & 4401.1 & 6054.8 & & & 5359.0 & 5094.8 & 5330.4 & 5705.7 & 6119.2 & 6581.8 & & 8841.5 & 15953.7 & \\
\hline Nominal dollar GDP growth & 14.1 & 29.1 & 37.6 & & & -11.5 & -4.9 & 4.6 & 7.0 & 7.2 & 7.6 & 1.7 & 6.1 & 6.1 & 6.1 \\
\hline PV of PPG external debt (in Millions of US dollars) & & & 900.8 & & & 1044.9 & 1298.0 & 1514.4 & 1713.4 & 1768.2 & 1799.2 & & 1976.8 & 3533.7 & \\
\hline (PVt-PVt-1)/GDPt-1 (in percent) & & & & & & 2.4 & 4.7 & 4.2 & 3.7 & 1.0 & 0.5 & 2.8 & 0.7 & 1.1 & 1.0 \\
\hline
\end{tabular}

Sources: Country authorities, and staff estimates and projections.

1/ Includes both public and private sector external debt.
$2 /$ Derived as $[\mathrm{r}-\mathrm{g}-\rho(1+\mathrm{g})](1+\mathrm{g}+\rho+\mathrm{g} \rho)$ times previous period debt ratio, with $\mathrm{r}=$ nominal interest rate; $\mathrm{g}=$ real GDP growth rate, and $\rho=$ growth rate of GDP deflator in U.S. dollar terms.

3 / Includes exceptional financing (i.e., changes in arrears and debt relief); changes in gross foreign assets; and valuation adjustments. For projections also includes contribution from price and exchange rate changes.

$5 /$ Current thear interest pate sector debt is equivalent to its face value.

6/ Historical averages and standard deviations are generally derived over the past 10 years, subject to data availability.

7/ Defined as grants, concessional loans, and debt relief.

8/ Grant-equivalent financing includes grants provided directly to the government and through new borrowing (difference between the face value and the PV of new debt). 
Table 2A.Moldova: Sensitivity Analysis for Key Indicators of Public and Publicly Guaranteed External Debt, 2009-2029 (In percent)

\begin{tabular}{|c|c|c|c|c|c|c|c|c|c|c|c|c|}
\hline & \multicolumn{12}{|c|}{ Projections } \\
\hline & 2009 & 2010 & 2011 & 2012 & 2013 & 2014 & 2015 & 2016 & 2017 & 2018 & 2019 & 2029 \\
\hline \multicolumn{13}{|c|}{ PV of debt-to GDP ratio } \\
\hline Baseline & 22 & 26 & 29 & 31 & 29 & 28 & 26 & 25 & 24 & 23 & 23 & 22 \\
\hline \multicolumn{13}{|l|}{ A. Alternative Scenarios } \\
\hline A1. Key variables at their historical averages in 2009-2029 1/ & 22 & 16 & 13 & 11 & 9 & 7 & 7 & 5 & 5 & 5 & 4 & 5 \\
\hline A2. New public sector loans on less favorable terms in 2009-2029 2 & 22 & 29 & 33 & 36 & 35 & 34 & 33 & 32 & 31 & 31 & 31 & 34 \\
\hline \multicolumn{13}{|l|}{ B. Bound Tests } \\
\hline B1. Real GDP growth at historical average minus one standard deviation in 2010-2011 & 22 & 24 & 26 & 27 & 25 & 22 & 20 & 18 & 16 & 15 & 14 & 14 \\
\hline B2. Export value growth at historical average minus one standard deviation in 2010-2011 3/ & 22 & 28 & 42 & 42 & 39 & 36 & 34 & 31 & 28 & 26 & 24 & 16 \\
\hline B3. US dollar GDP deflator at historical average minus one standard deviation in 2010-2011 & 22 & 24 & 28 & 28 & 26 & 24 & 21 & 19 & 17 & 16 & 15 & 15 \\
\hline B4. Net non-debt creating flows at historical average minus one standard deviation in 2010-2011 4/ & 22 & 35 & 49 & 48 & 46 & 42 & 40 & 37 & 33 & 30 & 28 & 17 \\
\hline B5. Combination of B1-B4 using one-half standard deviation shocks & 22 & 25 & 36 & 36 & 34 & 31 & 29 & 27 & 24 & 22 & 20 & 14 \\
\hline B6. One-time 30 percent nominal depreciation relative to the baseline in $20105 /$ & 22 & 35 & 38 & 39 & 36 & 32 & 29 & 26 & 23 & 22 & 20 & 21 \\
\hline
\end{tabular}

PV of debt-to-exports ratio

Baseline

\section{A. Alternative Scenarios}

A1. Key variables at their historical averages in 2009-2029 1/

A2. New public sector loans on less favorable terms in 2009-2029 2

\section{B. Bound Tests}

B1. Real GDP growth at historical average minus one standard deviation in 2010-2011

B2. Export value growth at historical average minus one standard deviation in 2010-2011 3/

B3. US dollar GDP deflator at historical average minus one standard devition in 2010-2011

B4. Net non-debt reting flows a tisticlave

B5. Combination of B1-B4 using one-half standard deviation shock

B6. One-time 30 percent nominal depreciation relative to the baseline in $20105 /$

\section{Baseline}

\section{A. Alternative Scenario}

A1. Key variables at their historical averages in 2009-2029 1/

A2. New public sector loans on less favorable terms in 2009-2029

\section{B. Bound Tests}

B1. Real GDP growth at historical average minus one standard deviation in 2010-2011

B2. Expot value

B3. US dollar GDP deflator at historical average minus one standard deviation in 2010-201

B4. Net non-debt creating flows at historical average minus one standard deviation in 2010-2011

B5. Combination of B1-B4 using one-half standard deviation shocks

B6. One-time 30 percent nominal depreciation relative to the baseline in $2010 \mathrm{~s} /$

61

$61 \quad 59$

61

$61 \quad 59$

$61 \quad 71$

61

PV of debt-to-revenue ratio

60

60

60

\section{0}

\section{1}

$\begin{array}{rrrrrrrrrrrr}60 & 68 & 71 & 71 & 63 & 56 & 51 & 45 & 41 & 38 & \mathbf{3 5} & 37 \\ 60 & 79 & 114 & 112 & 101 & 92 & 86 & 79 & 72 & 66 & \mathbf{6 1} & 40 \\ 60 & 67 & 76 & 76 & 67 & 60 & 54 & 48 & 44 & 40 & \mathbf{3 8} & 39 \\ 60 & 98 & 132 & 129 & 117 & 108 & 101 & 93 & 85 & 77 & \mathbf{7 1} & 42 \\ 60 & 71 & 98 & 96 & 87 & 79 & 74 & 68 & 62 & 56 & \mathbf{5 2} & 36 \\ 60 & 99 & 103 & 103 & 91 & 82 & 73 & 66 & 60 & 55 & \mathbf{5 1} & 53\end{array}$


Table 2A.Moldova: Sensitivity Analysis for Key Indicators of Public and Publicly Guaranteed External Debt, 2009-2029 (continued) (In percent)

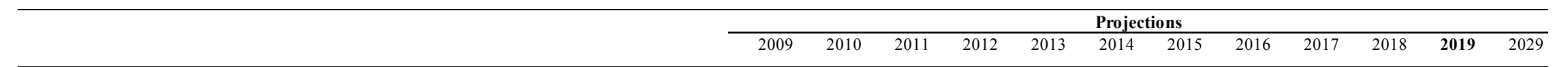

Debt service-to-exports ratio

Baseline

\section{A. Alternative Scenarios}

A1. Key variables at their historical averages in 2009-2029 1/

A2. New public sector loans on less favorable terms in 2009-2029 2

\section{B. Bound Tests}

B1. Real GDP growth at historical average minus one standard deviation in 2010-2011

B2. Export value growth at historical average minus one standard deviation in 2010-2011 3/

B3. US dollar GDP deflator at historical average minus one standard deviation in 2010-2011

B4. Net non-debt creating flows at historical average minus one standard deviation in 2010-2011 4 /

B5. Combination of B1-B4 using one-half standard deviation shocks

B6. One-time 30 percent nominal depreciation relative to the baseline in 2010 5/

$\begin{array}{lllllllllllll}5 & 4 & 3 & 4 & 4 & 3 & 3 & 4 & 5 & 5 & \mathbf{5} & 5 \\ & & & & & & & & & & & \\ 5 & 3 & 2 & 2 & 2 & 1 & 1 & 2 & 1 & 1 & \mathbf{1} & 1 \\ 5 & 4 & 4 & 4 & 4 & 4 & 4 & 5 & 5 & 5 & \mathbf{4} & 6 \\ & & & & & & & & & & & \\ 5 & 4 & 3 & 3 & 3 & 3 & 3 & 4 & 4 & 4 & \mathbf{4} & 3 \\ 5 & 5 & 5 & 6 & 6 & 5 & 5 & 7 & 9 & 9 & \mathbf{8} & 6 \\ 5 & 4 & 3 & 3 & 3 & 3 & 3 & 4 & 4 & 4 & \mathbf{4} & 3 \\ 5 & 4 & 4 & 5 & 5 & 4 & 4 & 6 & 8 & 7 & 7 & 4 \\ 5 & 4 & 4 & 5 & 5 & 4 & 4 & 6 & 7 & 7 & 7 & 5 \\ 5 & 4 & 3 & 3 & 3 & 3 & 3 & 4 & 4 & 4 & \mathbf{4} & 3\end{array}$

\section{Baseline}

\section{A. Alternative Scenarios}

A1. Key variables at their historical averages in 2009-2029 1/

A2. New public sector loans on less favorable terms in 2009-2029 2

\section{B. Bound Tests}

B1. Real GDP growth at historical average minus one standard deviation in 2010-2011

B2. Export value growth at historical average minus one standard deviation in 2010-2011 3/

B3. US dollar GDP deflator at historical average minus one standard deviation in 2010-2011

B4. Net non-debt creating flows at historical average minus one standard deviation in 2010-2011 4

B5. Combination of B1-B4 using one-half standard deviation shocks

B6. One-time 30 percent nominal depreciation relative to the baseline in 20105 /

Debt service-to-revenue ratio

Memorandum item:

Grant element assumed on residual financing (i.e., financing required above baseline) 6 /

Sources: Country authorities; and staff estimates and projections.

1/ Variables include real GDP growth, growth of GDP deflator (in U.S. dollar terms), non-interest current account in percent of GDP, and non-debt creating flows .

$2 /$ Assumes that the interest rate on new borrowing is by 2 percentage points higher than in the baseline., while grace and maturity periods are the same as in the baseline.

3/ Exports values are assumed to remain permanently at the lower level, but the current account as a share of GDP is assumed to return to its baseline level after the shock (implicitly assuming

an offsetting adjustment in import levels).

$4 /$ Includes official and private transfers and FDI.

5/ Depreciation is defined as percentage decline in dollar/local currency rate, such that it never exceeds 100 percent.

6/ Applies to all stress scenarios except for A2 (less favorable financing) in which the terms on all new financing are as specified in footnote 2. 
Table 3A.Moldova: Public Sector Debt Sustainability Framework, Baseline Scenario, 2006-2029

(In percent of GDP, unless otherwise indicated)

\begin{tabular}{|c|c|c|c|c|c|c|c|c|c|c|c|c|c|c|c|}
\hline & \multicolumn{3}{|c|}{ Actual } & \multirow[b]{2}{*}{ Average } & \multirow[b]{2}{*}{$\begin{array}{l}\text { Standard } \\
\text { Deviation }\end{array}$} & \multicolumn{5}{|l|}{ Estimate } & \multicolumn{5}{|c|}{ Projections } \\
\hline & 2006 & 2007 & 2008 & & & 2009 & 2010 & 2011 & 2012 & 2013 & 2014 & $\begin{array}{l}2009-14 \\
\text { Average }\end{array}$ & 2019 & 2029 & $\begin{array}{r}2015-29 \\
\text { Average }\end{array}$ \\
\hline $\begin{array}{l}\text { Public sector debt } 1 / \\
\text { o/w foreign-currency denominated }\end{array}$ & $\begin{array}{l}34.0 \\
25.6\end{array}$ & $\begin{array}{l}26.8 \\
19.8\end{array}$ & $\begin{array}{l}21.3 \\
15.7\end{array}$ & & & $\begin{array}{l}30.9 \\
22.4\end{array}$ & $\begin{array}{l}37.0 \\
28.7\end{array}$ & $\begin{array}{l}39.7 \\
32.3\end{array}$ & $\begin{array}{l}41.0 \\
34.6\end{array}$ & $\begin{array}{l}38.7 \\
33.1\end{array}$ & $\begin{array}{l}36.2 \\
31.3\end{array}$ & & $\begin{array}{l}29.7 \\
25.7\end{array}$ & $\begin{array}{l}25.5 \\
23.1\end{array}$ & \\
\hline Change in public sector debt & -3.6 & -7.2 & -5.5 & & & 9.6 & 6.0 & 2.7 & 1.3 & -2.3 & -2.5 & & -1.0 & -0.6 & \\
\hline Identified debt-creating flows & -6.0 & -8.5 & -6.3 & & & 12.9 & 5.1 & 2.4 & -0.3 & -2.3 & -2.0 & & -0.9 & -0.7 & \\
\hline Primary deficit & -1.3 & -1.0 & -0.2 & -2.0 & 3.5 & 7.5 & 5.5 & 3.6 & 1.7 & 0.3 & -0.2 & 3.1 & -0.1 & -0.2 & -0.2 \\
\hline Revenue and grants & 39.9 & 41.7 & 40.6 & & & 37.8 & 38.8 & 39.2 & 39.3 & 40.8 & 41.0 & & 41.0 & 41.0 & \\
\hline of which: grants & 0.7 & 1.8 & 1.7 & & & 1.9 & 3.2 & 2.5 & 1.8 & 1.7 & 1.6 & & 1.6 & 1.6 & \\
\hline Primary (noninterest) expenditure & 38.6 & 40.8 & 40.4 & & & 45.3 & 44.3 & 42.8 & 41.0 & 41.0 & 40.8 & & 40.8 & 40.7 & \\
\hline Automatic debt dynamics & -4.3 & -7.3 & -4.2 & & & 5.8 & 0.2 & -0.6 & -1.4 & -1.9 & -1.7 & & -0.8 & -0.4 & \\
\hline Contribution from interest rate/growth differential & -3.7 & -6.0 & -5.3 & & & 3.9 & 2.1 & -0.4 & -1.5 & -1.7 & -1.7 & & -0.8 & -0.4 & \\
\hline of which: contribution from average real interest rate & -2.0 & -5.0 & -3.4 & & & 1.7 & 2.5 & 0.6 & 0.4 & 0.3 & 0.1 & & 0.4 & 0.6 & \\
\hline of which: contribution from real GDP growth & -1.7 & -1.0 & -1.9 & & & 2.1 & -0.5 & -1.1 & -1.9 & -2.0 & -1.8 & & -1.2 & -1.0 & \\
\hline Contribution from real exchange rate depreciation & -0.6 & -1.3 & 1.1 & & & 2.0 & -1.9 & -0.2 & 0.1 & -0.2 & 0.0 & & $\ldots$ & $\ldots$ & \\
\hline Other identified debt-creating flows & -0.4 & -0.3 & -1.9 & & & -0.4 & -0.6 & -0.6 & -0.6 & -0.7 & -0.1 & & 0.0 & 0.0 & \\
\hline Privatization receipts (negative) & -0.4 & -0.3 & -1.9 & & & -0.4 & -0.6 & -0.6 & -0.6 & -0.7 & -0.1 & & 0.0 & 0.0 & \\
\hline Recognition of implicit or contingent liabilities & 0.0 & 0.0 & 0.0 & & & 0.0 & 0.0 & 0.0 & 0.0 & 0.0 & 0.0 & & 0.0 & 0.0 & \\
\hline Debt relief (HIPC and other) & 0.0 & 0.0 & 0.0 & & & 0.0 & 0.0 & 0.0 & 0.0 & 0.0 & 0.0 & & 0.0 & 0.0 & \\
\hline Other (specify, e.g. bank recapitalization) & 0.0 & 0.0 & 0.0 & & & 0.0 & 0.0 & 0.0 & 0.0 & 0.0 & 0.0 & & 0.0 & 0.0 & \\
\hline Residual, including asset changes & 2.3 & 1.3 & 0.8 & & & -3.3 & 0.9 & 0.4 & 1.6 & 0.0 & -0.5 & & 0.0 & 0.1 & \\
\hline \multicolumn{16}{|l|}{ Other Sustainability Indicators } \\
\hline PV of public sector debt & 8.5 & 7.0 & 20.5 & & & 30.2 & 34.3 & 36.2 & 37.0 & 34.9 & 32.6 & & 26.6 & 24.8 & \\
\hline $\mathrm{o} / \mathrm{w}$ foreign-currency denominated & 0.0 & 0.0 & 14.9 & & & 21.6 & 26.0 & 28.8 & 30.6 & 29.2 & 27.6 & & 22.6 & 22.4 & \\
\hline $\mathrm{o} / \mathrm{w}$ external & $\ldots$ & $\ldots$ & 14.9 & & & 21.6 & 26.0 & 28.8 & 30.6 & 29.2 & 27.6 & & 22.6 & 22.4 & \\
\hline PV of contingent liabilities (not included in public sector debt) & $\ldots$ & $\ldots$ & & & & $\ldots$ & $\ldots$ & $\ldots$ & & $\ldots$ & $\ldots$ & & $\ldots$ & $\ldots$ & \\
\hline Gross financing need $2 /$ & 6.9 & 4.8 & 4.9 & & & 12.9 & 15.2 & 13.3 & 10.7 & 8.7 & 7.3 & & 6.9 & 5.2 & \\
\hline $\mathrm{PV}$ of public sector debt-to-revenue and grants ratio (in percent) & 21.2 & 16.8 & 50.4 & & & 79.9 & 88.3 & 92.4 & 94.2 & 85.5 & 79.4 & & 64.9 & 60.6 & \\
\hline $\mathrm{PV}$ of public sector debt-to-revenue ratio (in percent) & 21.6 & 17.6 & 52.7 & & & 84.1 & 96.2 & 98.5 & 98.7 & 89.2 & 82.6 & & 67.5 & 63.0 & \\
\hline o/w external 3/ & & & 38.3 & & & 60.2 & 73.0 & 78.5 & 81.6 & 74.7 & 70.1 & & 57.3 & 56.8 & \\
\hline Debt service-to-revenue and grants ratio (in percent) $4 /$ & 9.2 & 6.9 & 6.0 & & & 7.2 & 6.8 & 7.0 & 7.0 & 6.6 & 6.0 & & 8.0 & 7.6 & \\
\hline Debt service-to-revenue ratio (in percent) $4 /$ & 9.3 & 7.2 & 6.3 & & & 7.6 & 7.4 & 7.4 & 7.3 & 6.9 & 6.2 & & 8.3 & 7.9 & \\
\hline Primary deficit that stabilizes the debt-to-GDP ratio & 2.3 & 6.2 & 5.3 & & & -2.1 & -0.5 & 0.8 & 0.3 & 2.6 & 2.3 & & 0.8 & 0.3 & \\
\hline \multicolumn{16}{|l|}{ Key macroeconomic and fiscal assumptions } \\
\hline Real GDP growth (in percent) & 4.8 & 3.0 & 7.8 & 5.0 & 3.6 & -9.0 & 1.5 & 3.0 & 5.0 & 5.0 & 5.0 & 1.8 & 4.0 & 4.0 & 4.0 \\
\hline Average nominal interest rate on forex debt (in percent) & 3.3 & 1.9 & 1.8 & 3.3 & 1.1 & 2.3 & 2.5 & 2.1 & 2.1 & 1.9 & 1.9 & 2.2 & 2.2 & 3.5 & 2.6 \\
\hline Average real interest rate on domestic debt (in percent) & -6.6 & -3.8 & 5.4 & -0.5 & 4.8 & 13.8 & 5.5 & 6.1 & 5.5 & 5.5 & $5.8^{\circ}$ & 7.0 & 7.6 & 10.0 & 8.3 \\
\hline Real exchange rate depreciation (in percent, + indicates depreciation) & -2.4 & -6.5 & 7.6 & -3.7 & 10.5 & 10.8 & $\ldots$ & & & $\ldots$ & $\ldots$ & $\ldots$ & $\ldots$ & $\ldots$ & $\ldots$ \\
\hline Inflation rate (GDP deflator, in percent) & 13.4 & 15.9 & 9.2 & 16.0 & 10.1 & 3.9 & 6.5 & 4.7 & 4.7 & 4.3 & 4.0 & 4.7 & 4.0 & 4.0 & 4.0 \\
\hline Growth of real primary spending (deflated by GDP deflator, in percent) & 0.1 & 0.1 & 0.1 & 0.1 & 0.2 & 0.0 & 0.0 & 0.0 & 0.0 & 0.1 & 0.0 & 0.0 & 0.0 & 0.0 & 0.0 \\
\hline Grant element of new external borrowing (in percent) & $\ldots$ & $\ldots$ & $\ldots$ & $\ldots$ & & -1.1 & 27.3 & 22.2 & 22.4 & 27.3 & 32.5 & 21.8 & 12.4 & -3.3 & $\ldots$ \\
\hline
\end{tabular}

Sources: Country authorities; and staff estimates and projection.

$2 /$ Gross financing need is defined as the primary deficit plus debt service plus the stock of short-term debt at the end of the last period.

3 Revenues excluding grants.

terest and amortization of medium and long-term deb.

5/ Historical averages and standard deviations are generally derived over the past 10 years, subject to data availability. 
Table 4A.Moldova: Sensitivity Analysis for Key Indicators of Public Debt 2009-2029

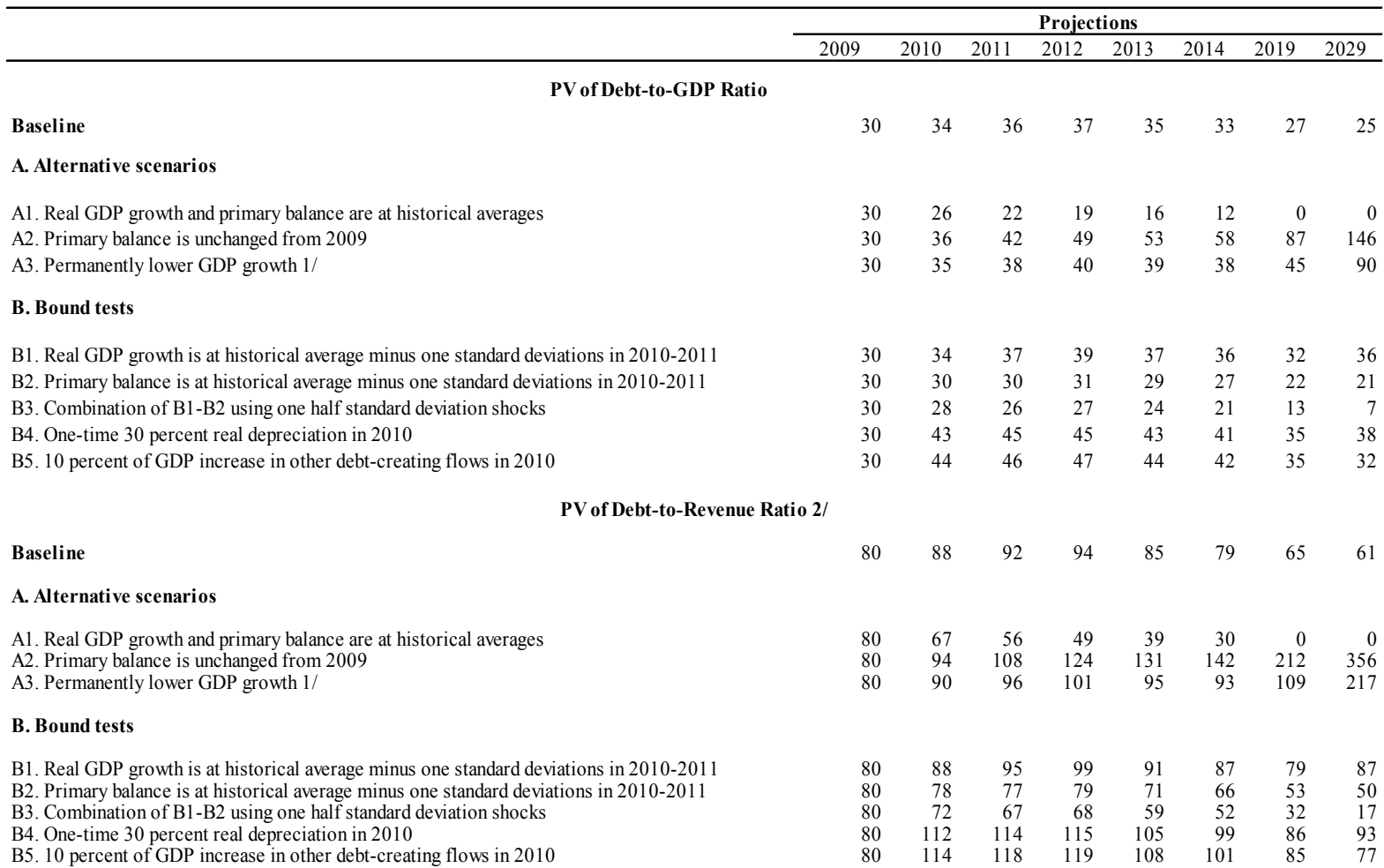

Debt Service-to-Revenue Ratio 2/

Baseline

\section{A. Alternative scenarios}

A1. Real GDP growth and primary balance are at historical averages

A2. Primary balance is unchanged from 2009

A3. Permanently lower GDP growth $1 /$

$\begin{array}{rrrrrrrr}7 & 7 & 6 & 5 & 4 & 4 & 2 & 0 \\ 7 & 7 & 7 & 8 & 8 & 8 & 17 & 34 \\ 7 & 7 & 7 & 7 & 7 & 7 & 11 & 20\end{array}$

B. Bound tests

B1. Real GDP growth is at historical average minus one standard deviations in 2010-2011

B2. Primary balance is at historical average minus one standard deviations in 2010-2011

B3. Combination of B1-B2 using one half standard deviation shocks

B4. One-time 30 percent real depreciation in 2010

B5. 10 percent of GDP increase in other debt-creating flows in 2010

$\begin{array}{rrrrrrrr}7 & 7 & 7 & 7 & 7 & 6 & 9 & 10 \\ 7 & 7 & 7 & 6 & 6 & 5 & 7 & 7 \\ 7 & 7 & 6 & 6 & 5 & 5 & 5 & 3 \\ 7 & 8 & 9 & 9 & 9 & 8 & 12 & 14 \\ 7 & 7 & 8 & 9 & 8 & 7 & 10 & 9\end{array}$

Sources: Country authorities; and staff estimates and projections.

1/ Assumes that real GDP growth is at baseline minus one standard deviation divided by the square root of the length of the projection period.

2/ Revenues are defined inclusive of grants. 
Table 5A: External Debt Sustainability Framework with Additional Non-Concessional Borrowing, and Positive Impact on Growth, 2009-2029 1/

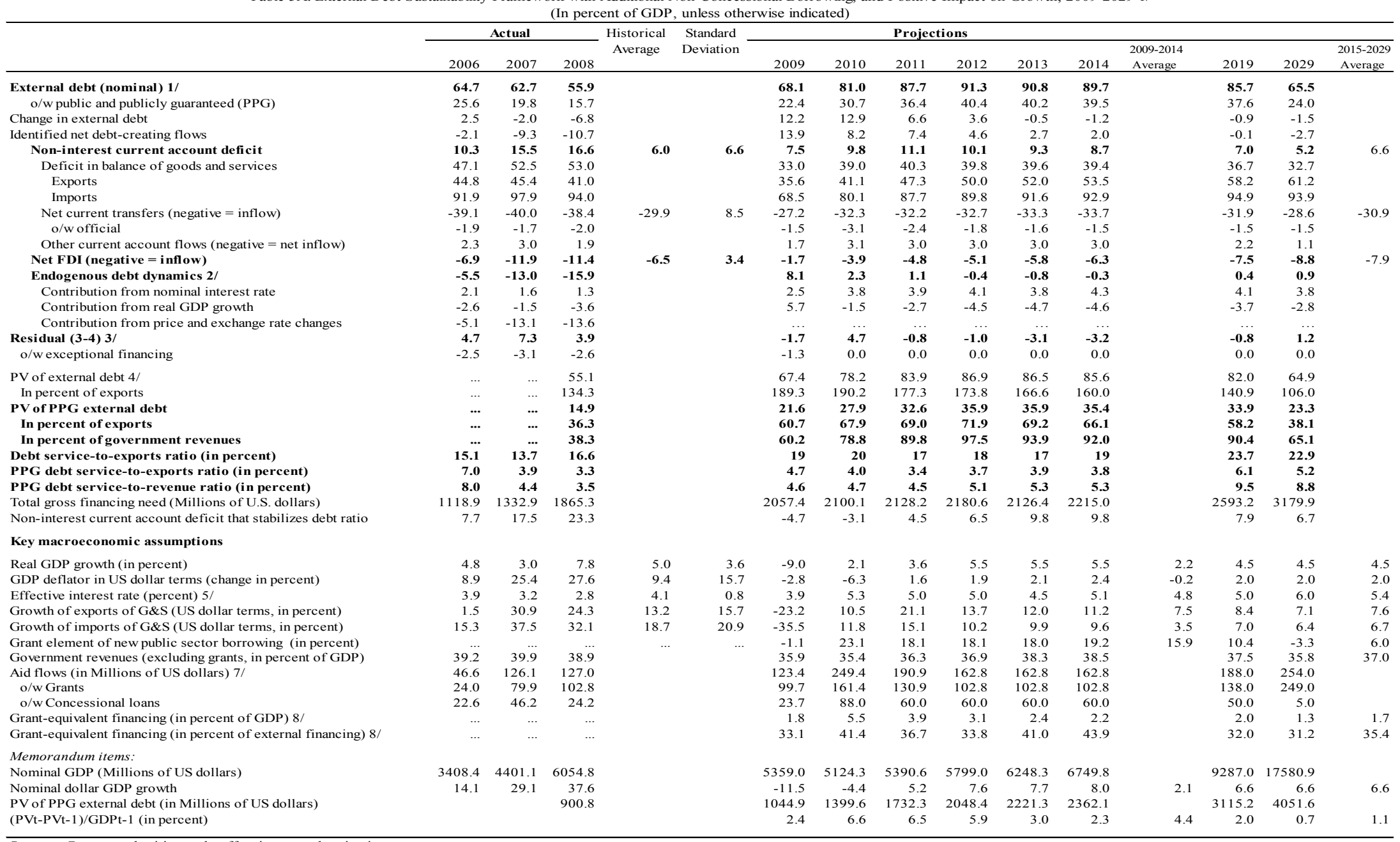

Sources: Country authorities; and staff estimates and projections

1/ Includes both public and private sector external debt.

2/ Derived as $[\mathrm{r}-\mathrm{g}-\rho(1+\mathrm{g})](1+\mathrm{g}+\rho+\mathrm{g} \rho)$ times previous period debt ratio, with $\mathrm{r}=$ nominal interest rate; $\mathrm{g}=$ real GDP growth rate, and $\rho=$ growth rate of GDP deflator in U.S. dollar terms.

3/ Includes exceptional financing (i.e., changes in arrears and debt relief); changes in gross foreign assets; and valuation adjustments. For projections also includes contribution from price and exchange rate changes.

4/ Assumes that PV of private sector debt is equivalent to its face value.

6/ Historical averages and standard deviations are generally derived over the past 10 years, subject to data availability.

8/ Grant-equivalent financing includes grants provided directly to the government and through new borrowing (difference between the face value and the PV of new debt). 
Table 6A.Moldova: Sensitivity Analysis for Key Indicators of Public and Publicly Guaranteed External Debt with Additional Non-Concessional Borrowing, and Positive Impact on Growth, 2009-2029 1/ (In percent)

\begin{tabular}{|c|c|c|c|c|c|c|c|c|c|c|c|c|}
\hline & \multicolumn{12}{|c|}{ Projections } \\
\hline & 2009 & 2010 & 2011 & 2012 & 2013 & 2014 & 2015 & 2016 & 2017 & 2018 & 2019 & 2029 \\
\hline \multicolumn{13}{|c|}{ PV of debt-to GDP ratio } \\
\hline Baseline & 22 & 28 & 33 & 36 & 36 & 35 & 35 & 34 & 34 & 34 & 34 & 23 \\
\hline \multicolumn{13}{|l|}{ A. Alternative Scenarios } \\
\hline A1. Key variables at their historical averages in 2009-2029 1/ & 22 & 16 & 13 & 10 & 6 & 3 & 1 & 0 & 0 & 0 & 1 & 9 \\
\hline A2. New public sector loans on less favorable terms in 2009-2029 2 & 22 & 32 & 38 & 42 & 43 & 43 & 43 & 43 & 44 & 44 & 45 & 37 \\
\hline \multicolumn{13}{|l|}{ B. Bound Tests } \\
\hline B1. Real GDP growth at historical average minus one standard deviation in 2010-2011 & 22 & 26 & 30 & 32 & 32 & 30 & 29 & 28 & 27 & 27 & 27 & 17 \\
\hline B2. Export value growth at historical average minus one standard deviation in 2010-2011 3/ & 22 & 30 & 47 & 48 & 47 & 45 & 43 & 41 & 39 & 37 & 35 & 18 \\
\hline B3. US dollar GDP deflator at historical average minus one standard deviation in 2010-2011 & 22 & 26 & 32 & 34 & 34 & 32 & 31 & 30 & 29 & 28 & 28 & 18 \\
\hline B4. Net non-debt creating flows at historical average minus one standard deviation in 2010-2011 4/ & 22 & 37 & 52 & 54 & 52 & 50 & 47 & 44 & 42 & 40 & 38 & 18 \\
\hline B5. Combination of B1-B4 using one-half standard deviation shocks & 22 & 28 & 41 & 43 & 42 & 40 & 38 & 36 & 34 & 33 & 31 & 16 \\
\hline B6. One-time 30 percent nominal depreciation relative to the baseline in 20105 / & 22 & 38 & 43 & 47 & 46 & 44 & 42 & 40 & 39 & 39 & 38 & 25 \\
\hline
\end{tabular}

\section{Baseline}

\section{A. Alternative Scenarios}

A1. Key variables at their historical averages in 2009-2029 $1 /$

A2. New public sector loans on less favorable terms in 2009-2029 2

\section{B. Bound Tests}

B1. Real GDP growth at historical average minus one standard deviation in 2010-2011

B2. Export value growth at historical average minus one standard deviation in 2010-2011 3/

B3. US dollar GDP deflator at historical average minus one standard deviation in 2010-2011

B4. Net non-debt creating flows at historical average minus one standard deviation in 2010-2011 4

B5. Combination of B1-B4 using one-half standard deviation shocks

B6. One-time 30 percent nominal depreciation relative to the baseline in $20105 /$

\section{Baseline}

A. Alternative Scenarios

A1. Key variables at their historical averages in 2009-2029 1/

A2. New public sector loans on less favorable terms in 2009-2029 2

\section{B. Bound Tests}

B1. Real GDP growth at historical average minus one standard deviation in 2010-2011

B2. Export value growth at historical average minus one standard deviation in 2010-20113/

B3. US dollar GDP deflator at historical average minus one standard deviation in 2010-2011

B4. Net non-debt creating flows at historical average minus one standard deviation in 2010-2011 4

B5. Combination of B1-B4 using one-half standard deviation shocks

B6. One-time 30 percent nominal depreciation relative to the baseline in 2010

\section{PV of debt-to-exports ratio}

$\begin{array}{rrrrrrrrrrrr}61 & 68 & 69 & 72 & 69 & 66 & 64 & 62 & 61 & 60 & \mathbf{5 8} & 38 \\ & & & & & & & & & & & \\ 61 & 40 & 27 & 20 & 12 & 6 & 3 & 0 & 0 & 0 & \mathbf{2} & 15 \\ 61 & 77 & 80 & 84 & 82 & 80 & 79 & 78 & 78 & 77 & \mathbf{7 7} & 60 \\ & & & & & & & & & & & \\ 61 & 63 & 62 & 63 & 60 & 55 & 52 & 49 & 47 & 46 & \mathbf{4 5} & 28 \\ 61 & 84 & 140 & 136 & 128 & 119 & 112 & 103 & 97 & 91 & \mathbf{8 6} & 42 \\ 61 & 63 & 62 & 63 & 60 & 55 & 52 & 49 & 47 & 46 & \mathbf{4 5} & 28 \\ 61 & 90 & 110 & 107 & 100 & 94 & 87 & 80 & 75 & 70 & \mathbf{6 5} & 30 \\ 61 & 77 & 115 & 113 & 106 & 98 & 92 & 85 & 80 & 76 & \mathbf{7 1} & 35 \\ 61 & 63 & 62 & 63 & 60 & 55 & 52 & 49 & 47 & 46 & \mathbf{4 5} & 28\end{array}$

PV of debt-to-revenue ratio

$\begin{array}{rrrrrrrrrrrr}60 & 79 & 90 & 98 & 94 & 92 & 91 & 90 & 90 & 90 & \mathbf{9 0} & 65 \\ & & & & & & & & & & & \\ 60 & 46 & 35 & 27 & 17 & 9 & 4 & 0 & 0 & 1 & \mathbf{3} & 25 \\ 60 & 90 & 104 & 114 & 112 & 111 & 112 & 113 & 115 & 117 & \mathbf{1 1 9} & 103 \\ & & & & & & & & & & & \\ 60 & 73 & 83 & 88 & 83 & 79 & 76 & 74 & 72 & 72 & \mathbf{7 1} & 49 \\ 60 & 86 & 129 & 131 & 123 & 118 & 113 & 107 & 102 & 98 & \mathbf{9 4} & 51 \\ 60 & 73 & 88 & 93 & 88 & 84 & 80 & 78 & 76 & 76 & \mathbf{7 5} & 51 \\ 60 & 104 & 144 & 145 & 136 & 130 & 124 & 117 & 111 & 106 & \mathbf{1 0 1} & 51 \\ 60 & 78 & 114 & 116 & 109 & 104 & 100 & 94 & 90 & 87 & \mathbf{8 4} & 46 \\ 60 & 107 & 119 & 126 & 119 & 113 & 109 & 105 & 104 & 103 & \mathbf{1 0 2} & 69\end{array}$


Table 6A.Moldova: Sensitivity Analysis for Key Indicators of Public and Publicly Guaranteed External Debt with Additional Non-Concessional Borrowing, and Positive Impact on Growth, 2009-2029 (continued) 1/ (In percent)

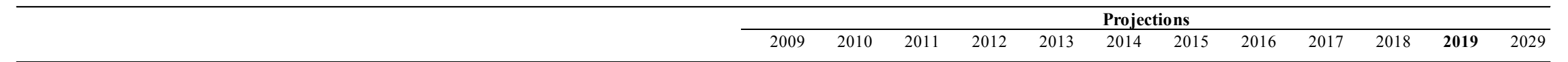

Debt service-to-exports ratio

\section{Baseline}

A. Alternative Scenarios

A1. Key variables at their historical averages in 2009-2029 1/

A2. New public sector loans on less favorable terms in 2009-2029 2

\section{B. Bound Tests}

B1. Real GDP growth at historical average minus one standard deviation in 2010-2011

B2. Export value growth at historical average minus one standard deviation in 2010-2011 3/

B3. US dollar GDP deflator at historical average minus one standard deviation in 2010-2011

B4. Net non-debt creating flows at historical average minus one standard deviation in 2010-2011 4

B5. Combination of B1-B4 using one-half standard deviation shocks

B6. One-time 30 percent nominal depreciation relative to the baseline in 2010 /

\section{Debt service-to-revenue ratio}

\section{Baseline}

\section{A. Alternative Scenarios}

A1. Key variables at their historical averages in 2009-2029 1/

A2. New public sector loans on less favorable terms in 2009-2029

\section{B. Bound Tests}

B1. Real GDP growth at historical average minus one standard deviation in 2010-2011

B2. Export value growth at historical average minus one standard deviation in 2010-2011 3/

B3. US dollar GDP deflator at historical average minus one standard deviation in 2010-2011

B4. Net non-debt creating flows at historical average minus one standard deviation in 2010-2011 4 /

B5. Combination of B1-B4 using one-half standard deviation shocks

B6. One-time 30 percent nominal depreciation relative to the baseline in $20105 /$

Memorandum item:

Grant element assumed on residual financing (i.e., financing required above baseline) 6/

$\begin{array}{rrrrrrrrrrrr}5 & 4 & 3 & 4 & 4 & 4 & 4 & 5 & 6 & 6 & \mathbf{6} & 5 \\ & & & & & & & & & & & \\ 5 & 3 & 2 & 2 & 2 & 1 & 1 & 1 & 1 & 1 & \mathbf{0} & 1 \\ 5 & 4 & 4 & 5 & 5 & 5 & 5 & 7 & 7 & 6 & \mathbf{6} & 7 \\ & & & & & & & & & & & \\ 5 & 4 & 3 & 4 & 4 & 4 & 4 & 5 & 5 & 5 & \mathbf{5} & 4 \\ 5 & 5 & 5 & 7 & 7 & 6 & 7 & 11 & 11 & 11 & \mathbf{1 0} & 6 \\ 5 & 4 & 3 & 4 & 4 & 4 & 4 & 5 & 5 & 5 & \mathbf{5} & 4 \\ 5 & 4 & 4 & 5 & 5 & 5 & 6 & 8 & 9 & 8 & \mathbf{8} & 4 \\ 5 & 4 & 4 & 6 & 6 & 5 & 6 & 9 & 9 & 9 & \mathbf{9} & 5 \\ 5 & 4 & 3 & 4 & 4 & 4 & 4 & 5 & 5 & 5 & \mathbf{5} & 4\end{array}$

\section{Sources: Country authorities; and staff estimates and projections.}

1/ Variables include real GDP growth, growth of GDP deflator (in U.S. dollar terms), non-interest current account in percent of GDP, and non-debt creating flows.

2/ Assumes that the interest rate on new borrowing is by 2 percentage points higher than in the baseline., while grace and maturity periods are the same as in the baseline.

3/ Exports values are assumed to remain permanently at the lower level, but the current account as a share of GDP is assumed to return to its baseline level after the shock (implicitly assuming

an offsetting adjustment in import levels).

4/ Includes official and private transfers and FDI

5/ Depreciation is defined as percentage decline in dollar/local currency rate, such that it never exceeds 100 percent.

6/ Applies to all stress scenarios except for A2 (less favorable financing) in which the terms on all new financing are as specified in footnote 2. 
Table 7A.Moldova: Public Sector Debt Sustainability Framework, Baseline Scenario with Additional Non-Concessional Borrowing, and Positive Impact on Growth, 2009-2029 1/ (In percent of GDP, unless otherwise indicated)

\begin{tabular}{|c|c|c|c|c|c|c|c|c|c|c|c|c|c|c|c|}
\hline & \multicolumn{3}{|c|}{ Actual } & \multirow[b]{2}{*}{ Average } & \multirow[b]{2}{*}{$\begin{array}{l}\text { Standard } \\
\text { Deviation }\end{array}$} & \multicolumn{5}{|l|}{ Estimate } & \multicolumn{5}{|c|}{ Projections } \\
\hline & 2006 & 2007 & 2008 & & & 2009 & 2010 & 2011 & 2012 & 2013 & 2014 & $\begin{array}{l}2009-14 \\
\text { Average }\end{array}$ & 2019 & 2029 & $\begin{array}{l}2015-29 \\
\text { Average } \\
\end{array}$ \\
\hline $\begin{array}{l}\text { Public sector debt } 1 / \\
\text { o/w foreign-currency denominated }\end{array}$ & $\begin{array}{l}34.0 \\
25.6\end{array}$ & $\begin{array}{l}26.8 \\
19.8\end{array}$ & $\begin{array}{l}21.3 \\
15.7\end{array}$ & & & $\begin{array}{l}30.9 \\
22.4\end{array}$ & $\begin{array}{l}38.9 \\
30.7\end{array}$ & $\begin{array}{l}43.7 \\
36.4\end{array}$ & $\begin{array}{l}46.7 \\
40.4\end{array}$ & $\begin{array}{l}45.8 \\
40.2\end{array}$ & $\begin{array}{l}44.3 \\
39.5\end{array}$ & & $\begin{array}{l}41.4 \\
37.6\end{array}$ & $\begin{array}{l}26.2 \\
24.0\end{array}$ & \\
\hline Change in public sector debt & -3.6 & -7.2 & -5.5 & & & 9.6 & 8.0 & 4.7 & 3.0 & -0.9 & -1.5 & & -0.5 & -1.2 & \\
\hline Identified debt-creating flows & -6.0 & -8.5 & -6.3 & & & 12.9 & 4.9 & 2.0 & -0.8 & -3.0 & -2.7 & & -1.8 & -0.9 & \\
\hline Primary deficit & -1.3 & -1.0 & -0.2 & -2.0 & 3.5 & 7.5 & 5.5 & 3.5 & 1.5 & 0.1 & -0.4 & 2.9 & -0.5 & -0.3 & -0.4 \\
\hline Revenue and grants & 39.9 & 41.7 & 40.6 & & & 37.8 & 38.6 & 38.8 & 38.6 & 39.9 & 40.0 & & 39.0 & 37.2 & \\
\hline of which: grants & 0.7 & 1.8 & 1.7 & & & 1.9 & 3.1 & 2.4 & 1.8 & 1.6 & 1.5 & & 1.5 & 1.4 & \\
\hline Primary (noninterest) expenditure & 38.6 & 40.8 & 40.4 & & & 45.3 & 44.0 & 42.2 & 40.2 & 40.0 & 39.6 & & 38.5 & 36.8 & \\
\hline Automatic debt dynamics & -4.3 & -7.3 & -4.2 & & & 5.8 & 0.0 & -0.9 & -1.7 & -2.4 & -2.2 & & -1.4 & -0.6 & \\
\hline Contribution from interest rate/growth differential & -3.7 & -6.0 & -5.3 & & & 3.9 & 1.9 & -0.7 & -1.8 & -2.1 & -2.2 & & -1.4 & -0.6 & \\
\hline of which: contribution from average real interest rate & -2.0 & -5.0 & -3.4 & & & 1.7 & 2.5 & 0.7 & 0.5 & 0.3 & 0.2 & & 0.5 & 0.6 & \\
\hline of which: contribution from real GDP growth & -1.7 & -1.0 & -1.9 & & & 2.1 & -0.6 & -1.3 & -2.3 & -2.4 & -2.4 & & -1.8 & -1.2 & \\
\hline Contribution from real exchange rate depreciation & -0.6 & -1.3 & 1.1 & & & 2.0 & -1.9 & -0.2 & 0.1 & -0.3 & 0.0 & & $\ldots$ & $\ldots$ & \\
\hline Other identified debt-creating flows & -0.4 & -0.3 & -1.9 & & & -0.4 & -0.6 & -0.6 & -0.6 & -0.7 & -0.1 & & 0.0 & 0.0 & \\
\hline Privatization receipts (negative) & -0.4 & -0.3 & -1.9 & & & -0.4 & -0.6 & -0.6 & -0.6 & -0.7 & -0.1 & & 0.0 & 0.0 & \\
\hline Recognition of implicit or contingent liabilities & 0.0 & 0.0 & 0.0 & & & 0.0 & 0.0 & 0.0 & 0.0 & 0.0 & 0.0 & & 0.0 & 0.0 & \\
\hline Debt relief (HIPC and other) & 0.0 & 0.0 & 0.0 & & & 0.0 & 0.0 & 0.0 & 0.0 & 0.0 & 0.0 & & 0.0 & 0.0 & \\
\hline Other (specify, e.g. bank recapitalization) & 0.0 & 0.0 & 0.0 & & & 0.0 & 0.0 & 0.0 & 0.0 & 0.0 & 0.0 & & 0.0 & 0.0 & \\
\hline Residual, including asset changes & 2.3 & 1.3 & 0.8 & & & -3.3 & 3.1 & 2.7 & 3.8 & 2.0 & 1.3 & & 1.3 & -0.2 & \\
\hline \multicolumn{16}{|l|}{ Other Sustainability Indicators } \\
\hline PV of public sector debt & 8.5 & 7.0 & 20.5 & & & 30.2 & 36.1 & 39.9 & 42.3 & 41.5 & 40.2 & & 37.7 & 25.5 & \\
\hline $\mathrm{o} / \mathrm{w}$ foreign-currency denominated & 0.0 & 0.0 & 14.9 & & & 21.6 & 27.9 & 32.6 & 35.9 & 35.9 & 35.4 & & 33.9 & 23.3 & \\
\hline $\mathrm{o} / \mathrm{w}$ external & $\ldots$ & $\ldots$ & 14.9 & & & 21.6 & 27.9 & 32.6 & 35.9 & 35.9 & 35.4 & & 33.9 & 23.3 & \\
\hline PV of contingent liabilities (not included in public sector debt) & $\ldots$ & $\ldots$ & $\ldots$ & & & $\ldots$ & & $\ldots$ & ... & $\ldots$ & $\ldots$ & & $\ldots$ & & \\
\hline Gross financing need $2 /$ & 6.9 & 4.8 & 4.9 & & & 12.9 & 15.1 & 13.1 & 10.6 & 8.5 & 7.2 & & 7.2 & 5.3 & \\
\hline $\begin{array}{l}\mathrm{PV} \text { of public sector debt-to-revenue and grants ratio (in percent) } \\
\text { (a) }\end{array}$ & 21.2 & 16.8 & 50.4 & & & 79.9 & 93.6 & 103.0 & 109.4 & 103.8 & 100.5 & & 96.7 & 68.6 & \\
\hline $\mathrm{PV}$ of public sector debt-to-revenue ratio (in percent) & 21.6 & 17.6 & 52.7 & & & 84.1 & 101.9 & 109.8 & 114.7 & 108.3 & 104.5 & & 100.5 & 71.3 & \\
\hline o/w external 3/ & & & 38.3 & & & 60.2 & 78.8 & 89.8 & 97.5 & 93.9 & 92.0 & & 90.4 & 65.1 & \\
\hline Debt service-to-revenue and grants ratio (in percent) $4 /$ & 9.2 & 6.9 & 6.0 & & & 7.2 & 6.8 & 7.1 & 7.3 & 7.1 & 6.9 & & 10.6 & 9.5 & \\
\hline Debt service-to-revenue ratio (in percent) $4 /$ & 9.3 & 7.2 & 6.3 & & & 7.6 & 7.4 & 7.6 & 7.6 & 7.4 & 7.1 & & 11.0 & 9.9 & \\
\hline Primary deficit that stabilizes the debt-to-GDP ratio & 2.3 & 6.2 & 5.3 & & & -2.1 & -2.5 & -1.3 & -1.5 & 1.0 & 1.0 & & 0.1 & 0.9 & \\
\hline \multicolumn{16}{|l|}{ Key macroeconomic and fiscal assumptions } \\
\hline Real GDP growth (in percent) & 4.8 & 3.0 & 7.8 & 5.0 & 3.6 & -9.0 & 2.1 & 3.6 & 5.5 & 5.5 & 5.5 & 2.2 & 4.5 & 4.5 & 4.5 \\
\hline Average nominal interest rate on forex debt (in percent) & 3.3 & 1.9 & 1.8 & 3.3 & 1.1 & 2.3 & 2.5 & 2.2 & 2.2 & 2.1 & 2.1 & 2.2 & 2.5 & $3.5^{\circ}$ & 2.8 \\
\hline Average real interest rate on domestic debt (in percent) & -6.6 & -3.8 & 5.4 & -0.5 & 4.8 & 13.8 & 5.5 & 6.1 & 5.5 & 5.5 & $5.8^{\circ}$ & 7.0 & 7.6 & $10.0^{\circ}$ & 8.3 \\
\hline Real exchange rate depreciation (in percent, + indicates depreciation) & -2.4 & -6.5 & 7.6 & -3.7 & 10.5 & 10.8 & $\ldots$ & $\ldots$ & $\ldots$ & & $\ldots$ & $\ldots$ & $\ldots$ & $\ldots$ & $\ldots$ \\
\hline Inflation rate (GDP deflator, in percent) & 13.4 & 15.9 & 9.2 & 16.0 & 10.1 & 3.9 & 6.5 & 4.7 & 4.7 & 4.3 & 4.0 & 4.7 & 4.0 & 4.0 & 4.0 \\
\hline Growth of real primary spending (deflated by GDP deflator, in percent) & 0.1 & 0.1 & 0.1 & 0.1 & 0.2 & 0.0 & 0.0 & 0.0 & 0.0 & 0.1 & 0.0 & 0.0 & 0.0 & 0.0 & 0.0 \\
\hline Grant element of new external borrowing (in percent) & $\ldots$ & $\ldots$ & $\ldots$ & & & -1.1 & 23.1 & 18.1 & 18.1 & 18.0 & 19.2 & 15.9 & 10.4 & -3.3 & \\
\hline
\end{tabular}

Sources: Country authorities; and staff estimates and projections.

/ Gross financing need is defined as the primary deficit plus debt service plus the stock of short-term debt at the end of the last period.

3/ Revenues excluding grants.

(1)

$5 /$ Historical averages and standard deviations are generally derived over the past 10 years, subject to data availability. 
Table 8A.Moldova: Sensitivity Analysis for Key Indicators of Public Debt 2009-2029

\begin{tabular}{|c|c|c|c|c|c|c|c|c|}
\hline & \multicolumn{8}{|c|}{ Projections } \\
\hline & 2009 & 2010 & 2011 & 2012 & 2013 & 2014 & 2019 & 2029 \\
\hline \multicolumn{9}{|c|}{ PV of Debt-to-GDP Ratio } \\
\hline Baseline & 30 & 36 & 40 & 42 & 41 & 40 & 38 & 26 \\
\hline \multicolumn{9}{|l|}{ A. Alternative scenarios } \\
\hline A1. Real GDP growth and primary balance are at historical averages & 30 & 28 & 26 & 25 & 23 & 21 & 13 & 0 \\
\hline A2. Primary balance is unchanged from 2009 & 30 & 38 & 46 & 54 & 60 & 66 & 99 & 145 \\
\hline A3. Permanently lower GDP growth $1 /$ & 30 & 37 & 41 & 45 & 45 & 46 & 56 & 84 \\
\hline \multicolumn{9}{|l|}{ B. Bound tests } \\
\hline B1. Real GDP growth is at historical average minus one standard deviations in $2010-2011$ & 30 & 37 & 42 & 46 & 46 & 46 & 48 & 42 \\
\hline B2. Primary balance is at historical average minus one standard deviations in $2010-2011$ & 30 & 32 & 34 & 37 & 36 & 35 & 33 & 22 \\
\hline B3. Combination of B1-B2 using one half standard deviation shocks & 30 & 30 & 30 & 33 & 32 & 31 & 28 & 16 \\
\hline B4. One-time 30 percent real depreciation in 2010 & 30 & 45 & 48 & 50 & 49 & 48 & 47 & 41 \\
\hline B5. 10 percent of GDP increase in other debt-creating flows in 2010 & 30 & 46 & 50 & 52 & 51 & 49 & 46 & 32 \\
\hline
\end{tabular}

PV of Debt-to-Revenue Ratio 2/

Baseline

$\begin{array}{lllllll}80 & 94 & 103 & 109 & 104 & 101 & 97\end{array}$

69

A. Alternative scenarios

A1. Real GDP growth and primary balance are at historical averages

A2. Primary balance is unchanged from 2009

A3. Permanently lower GDP growth $1 /$

$\begin{array}{rrrrrrrr}80 & 72 & 67 & 65 & 58 & 53 & 33 & 0 \\ 80 & 99 & 119 & 140 & 151 & 166 & 254 & 391\end{array}$

B. Bound tests

B1. Real GDP growth is at historical average minus one standard deviations in 2010-2011

B2. Primary balance is at historical average minus one standard deviations in 2010-2011

B3. Combination of B1-B2 using one half standard deviation shocks

B4. One-time 30 percent real depreciation in 2010

B5. 10 percent of GDP increase in other debt-creating flows in 2010

Debt Service-to-Revenue Ratio 2/

Baseline

\section{A. Alternative scenarios}

A1. Real GDP growth and primary balance are at historical averages

A2. Primary balance is unchanged from 2009

A3. Permanently lower GDP growth $1 /$

$\begin{array}{rrrrrrrr}7 & 7 & 6 & 5 & 5 & 5 & 5 & 1 \\ 7 & 7 & 7 & 8 & 9 & 9 & 20 & 38 \\ 7 & 7 & 7 & 8 & 7 & 7 & 13 & 22\end{array}$

\section{B. Bound test}

B1. Real GDP growth is at historical average minus one standard deviations in 2010-2011

B2. Primary balance is at historical average minus one standard deviations in 2010-2011

B3. Combination of B1-B2 using one half standard deviation shocks

B4. One-time 30 percent real depreciation in 2010

B5. 10 percent of GDP increase in other debt-creating flows in 2010

\begin{tabular}{rrrrrrrr}
7 & 7 & 7 & 8 & 8 & 7 & 12 & 14 \\
7 & 7 & 7 & 6 & 6 & 6 & 9 & 8 \\
7 & 7 & 6 & 6 & 6 & 6 & 8 & 7 \\
7 & 8 & 9 & 10 & 10 & 10 & 16 & 19 \\
7 & 7 & 8 & 9 & 8 & 8 & 13 & 11 \\
\hline
\end{tabular}

Sources: Country authorities; and staff estimates and projections.

1/ Assumes that real GDP growth is at baseline minus one standard deviation divided by the square root of the length of the projection period.

2/ Revenues are defined inclusive of grants. 
Table 9A. Moldova: External Debt Sustainability Framework with Additional Non-Concessional Borrowing and no Impact on Growth, 2006-2029 1/

\begin{tabular}{|c|c|c|c|c|c|c|c|c|c|c|c|c|c|c|c|}
\hline & \multicolumn{3}{|c|}{ Actual } & \multirow{3}{*}{$\begin{array}{l}\text { Historical } \\
\text { Average }\end{array}$} & \multirow{3}{*}{$\begin{array}{c}\text { Standard } \\
\text { Deviation }\end{array}$} & \multicolumn{6}{|c|}{ Projections } & \multirow[b]{2}{*}{$2009-2014$} & \multirow{3}{*}{2019} & \multirow{3}{*}{2029} & \multirow{3}{*}{$\begin{array}{c}2015-2029 \\
\text { Average }\end{array}$} \\
\hline & & & & & & & & & & & & & & & \\
\hline & 2006 & 2007 & 2008 & & & 2009 & 2010 & 2011 & 2012 & 2013 & 2014 & Average & & & \\
\hline External debt (nominal) 1/ & 64.7 & 62.7 & 55.9 & & & 68.1 & 81.5 & 88.7 & 92.8 & 92.8 & 92.0 & & 87.8 & 69.5 & \\
\hline $\mathrm{o} / \mathrm{w}$ public and publicly guaranteed $(\mathrm{PPG})$ & 25.6 & 19.8 & 15.7 & & & 22.4 & 30.9 & 36.8 & 41.0 & 41.1 & 40.5 & & 37.3 & 25.9 & \\
\hline Change in external debt & 2.5 & -2.0 & -6.8 & & & 12.2 & 13.4 & 7.2 & 4.1 & 0.0 & -0.8 & & -1.6 & -1.4 & \\
\hline Identified net debt-creating flows & -2.1 & -9.3 & -10.7 & & & 13.9 & 8.3 & 7.2 & 3.9 & 1.5 & 0.4 & & 1.0 & 1.2 & \\
\hline Non-interest current account deficit & 10.3 & 15.5 & 16.6 & 6.0 & 6.6 & 7.5 & 9.4 & 10.5 & 9.0 & 7.8 & 6.8 & & 6.9 & 7.1 & 7.0 \\
\hline Deficit in balance of goods and services & 47.1 & 52.5 & 53.0 & & & 33.0 & 38.8 & 39.9 & 39.1 & 38.7 & 38.3 & & 37.4 & 35.4 & \\
\hline Exports & 44.8 & 45.4 & 41.0 & & & 35.6 & 41.0 & 47.2 & 49.8 & 51.7 & 53.0 & & 55.5 & 58.4 & \\
\hline Imports & 91.9 & 97.9 & 94.0 & & & 68.5 & 79.9 & 87.1 & 88.9 & 90.4 & 91.3 & & 93.0 & 93.8 & \\
\hline Net current transfers (negative $=$ inflow) & -39.1 & -40.0 & -38.4 & -29.9 & 8.5 & -27.2 & -32.4 & -32.5 & -33.1 & -33.9 & -34.5 & & -32.6 & -29.2 & -31.6 \\
\hline o/w official & -1.9 & -1.7 & -2.0 & & & -1.5 & -3.2 & -2.5 & -1.8 & -1.7 & -1.6 & & -1.6 & -1.6 & \\
\hline Net FDI (negative $=$ inflow) & -6.9 & -11.9 & -11.4 & -6.5 & 3.4 & -1.7 & -3.9 & -4.9 & -5.2 & -6.0 & -6.4 & & -6.8 & -7.2 & -6.9 \\
\hline Endogenous debt dynamics 2/ & -5.5 & -13.0 & -15.9 & & & 8.1 & 2.7 & 1.6 & 0.0 & -0.4 & 0.1 & & 0.9 & 1.3 & \\
\hline Contribution from nominal interest rate & 2.1 & 1.6 & 1.3 & & & 2.5 & 3.8 & 3.9 & 4.2 & 3.9 & 4.4 & & 4.2 & 3.9 & \\
\hline Contribution from real GDP growth & -2.6 & -1.5 & -3.6 & & & 5.7 & -1.1 & -2.3 & -4.1 & -4.3 & -4.3 & & -3.4 & -2.7 & \\
\hline Contribution from price and exchange rate changes & -5.1 & -13.1 & -13.6 & & & & & & & & & & 0.7 & & \\
\hline Residual (3-4) 3/ & 4.7 & 7.3 & 3.9 & & & -1.7 & 5.1 & 0.0 & 0.3 & -1.5 & -1.2 & & -2.6 & -2.6 & \\
\hline $\mathrm{o} / \mathrm{w}$ exceptional financing & -2.5 & -3.1 & -2.6 & & & -1.3 & 0.0 & 0.0 & 0.0 & 0.0 & 0.0 & & 0.0 & 0.0 & \\
\hline PV of external debt 4/ & $\ldots$ & $\ldots$ & 55.1 & & & 67.4 & 78.7 & 84.9 & 88.3 & 88.4 & 87.7 & & 84.0 & 68.6 & \\
\hline In percent of exports & $\ldots$ & $\ldots$ & 134.3 & & & 189.3 & 191.7 & 179.6 & 177.2 & 171.1 & 165.5 & & 151.2 & 117.5 & \\
\hline PV of PPG external debt & $\ldots$ & $\ldots$ & 14.9 & & & 21.6 & 28.1 & 33.0 & 36.5 & 36.7 & 36.3 & & 33.5 & 25.1 & \\
\hline In percent of exports & $\ldots$ & $\ldots$ & 36.3 & & & 60.7 & 68.4 & 69.8 & 73.3 & 71.0 & 68.4 & & 60.3 & 42.9 & \\
\hline In percent of government revenues & ... & ... & 38.3 & & & 60.2 & 78.2 & 89.8 & 97.5 & 93.9 & 92.0 & & 85.1 & 63.6 & \\
\hline Debt service-to-exports ratio (in percent) & 15.1 & 13.7 & 16.6 & & & 19 & 21 & 17 & 19 & 17 & 20 & & 26.2 & 24.9 & \\
\hline Total gross financing need (Millions of U.S. dollars) & 1118.9 & 1332.9 & 1865.3 & & & 2057.4 & 2078.7 & 2086.2 & 2108.5 & 2022.9 & 2079.0 & & 2659.3 & 3679.6 & \\
\hline Non-interest current account deficit that stabilizes debt ratio & 7.7 & 17.5 & 23.3 & & & -4.7 & -3.9 & 3.3 & 4.9 & 7.9 & 7.6 & & 8.5 & 8.5 & \\
\hline \multicolumn{16}{|l|}{ Key macroeconomic assumptions } \\
\hline Real GDP growth (in percent) & 4.8 & 3.0 & 7.8 & 5.0 & 3.6 & -9.0 & 1.5 & 3.0 & 5.0 & 5.0 & 5.0 & 1.8 & 4.0 & 4.0 & 4.0 \\
\hline GDP deflator in US dollar terms (change in percent) & 8.9 & 25.4 & 27.6 & 9.4 & 15.7 & -2.8 & -6.3 & 1.6 & 1.9 & 2.1 & 2.4 & -0.2 & 2.0 & 2.0 & 2.0 \\
\hline Effective interest rate (percent) $5 /$ & 3.9 & 3.2 & 2.8 & 4.1 & 0.8 & 3.9 & 5.3 & 5.0 & 5.0 & 4.5 & 5.1 & 4.8 & 5.0 & 5.9 & 5.4 \\
\hline Growth of exports of G\&S (US dollar terms, in percent) & 1.5 & 30.9 & 24.3 & 13.2 & 15.7 & -23.2 & 9.6 & 20.4 & 12.9 & 11.2 & 10.4 & 6.9 & 6.6 & 6.6 & 6.8 \\
\hline Growth of imports of G\&S (US dollar terms, in percent) & 15.3 & 37.5 & 32.1 & 18.7 & 20.9 & -35.5 & 10.8 & 14.2 & 9.3 & 9.0 & 8.7 & 2.7 & 6.3 & 6.2 & 6.3 \\
\hline Grant element of new public sector borrowing (in percent) & & & & & $\ldots$ & -1.1 & 23.1 & 18.1 & 18.1 & 18.0 & 19.2 & 15.9 & 11.5 & -2.4 & 6.6 \\
\hline Government revenues (excluding grants, in percent of GDP) & 39.2 & 39.9 & 38.9 & & & 35.9 & 35.9 & 36.7 & 37.5 & 39.1 & 39.4 & & 39.4 & 39.4 & 39.4 \\
\hline Aid flows (in Millions of US dollars) $7 /$ & 46.6 & 126.1 & 127.0 & & & 123.4 & 249.4 & 191.0 & 162.8 & 162.8 & 162.8 & & 188.0 & 254.1 & \\
\hline $\mathrm{o} / \mathrm{w}$ Grants & 24.0 & 79.9 & 102.8 & & & 99.7 & 161.4 & 131.0 & 102.8 & 102.8 & 102.8 & & 138.0 & 249.1 & \\
\hline $\mathrm{o} / \mathrm{w}$ Concessional loans & 22.6 & 46.2 & 24.2 & & & 23.7 & 88.0 & 60.0 & 60.0 & 60.0 & 60.0 & & 50.0 & 5.0 & \\
\hline Grant-equivalent financing (in percent of GDP) $8 /$ & ... & & $\ldots$ & & & 1.8 & 5.5 & 4.0 & 3.2 & 2.5 & 2.2 & & 2.0 & 1.5 & 1.8 \\
\hline Grant-equivalent financing (in percent of external financing) $8 /$ & $\ldots$ & $\ldots$ & $\ldots$ & & & 33.1 & 41.4 & 36.7 & 33.8 & 41.0 & 43.9 & & 35.8 & 32.2 & 36.6 \\
\hline \multicolumn{16}{|l|}{ Memorandum items: } \\
\hline Nominal GDP (Millions of US dollars) & 3408.4 & 4401.1 & 6054.8 & & & 5359.0 & 5094.8 & 5330.4 & 5705.7 & 6119.2 & 6581.8 & & 8841.5 & 15953.7 & \\
\hline Nominal dollar GDP growth & 14.1 & 29.1 & 37.6 & & & -11.5 & -4.9 & 4.6 & 7.0 & 7.2 & 7.6 & 1.7 & 6.1 & 6.1 & 6.1 \\
\hline PV of PPG external debt (in Millions of US dollars) & & & 900.8 & & & 1044.9 & 1399.6 & 1732.3 & 2048.4 & 2221.3 & 2362.1 & & 2931.3 & 3955.0 & \\
\hline (PVt-PVt-1)/GDPt-1 (in percent) & & & & & & 2.4 & 6.6 & 6.5 & 5.9 & 3.0 & 2.3 & 4.5 & 1.3 & 0.9 & 1.1 \\
\hline
\end{tabular}

Sources: Country authorities; and staff estimates and projections

1/ Includes both public and private sector external debt.
$2 /$ Derived as $[r-g-\rho(1+g)](1+g+\rho+g \rho)$ times previous period debt ratio, with $r=$ nominal interest rate; $g=$ real GDP growth rate, and $\rho=$ growth rate of GDP deflator in U.S. dollar term

3/ Includes exceptional financing (i.e., changes in arrears and debt relief); changes in gross foreign assets; and valuation adjustments. For projections also includes contribution from price and exchange rate changes.

4/ Assumes that $P$ or private sector debt is equivalent to its face value.

$6 /$ Historical averages and standard deviations are generally derived over the past 10 years, subject to data availability.

8/ Grant-equivalent financing includes grants provided directly to the government and through new borrowing (difference between the face value and the PV of new debt) 
Table 10A.Moldova: Sensitivity Analysis for Key Indicators of Public and Publicly Guaranteed External Debt with Additional Non-Concessional Borrowing and no Impact on Growth, 2009-2029 (In percent)

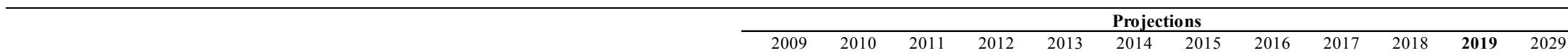

\section{PV of debt-to GDP ratio}

\section{Baseline}

\section{A. Alternative Scenarios}

A1. Key variables at their historical averages in 2009-2029 1/

A2. New public sector loans on less favorable terms in 2009-2029 2

\section{B. Bound Tests} B2. Export value growth at historical average minus one standard deviation in 2010-2011 3 / B3. US dollar GDP deflator at historical average minus one standard deviation in 2010-2011 B5. Combination of B1-B4 using one-half standard deviation shocks

B6. One-time 30 percent nominal depreciation relative to the baseline in $20105 /$
B1. Real GDP growth at historical average minus one standard deviation in 2010-201 B4. Net non-debt creating flows at historical average minus one standard deviation in 2010-2011 4 /

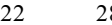

$\begin{array}{lllll}22 & 17 & 14 & 12 & 10 \\ 22 & 32 & 38 & 43 & 44 \\ & & & & \\ 22 & 26 & 30 & 33 & 32 \\ 22 & 30 & 47 & 48 & 47 \\ 22 & 26 & 32 & 35 & 34 \\ 22 & 37 & 53 & 55 & 54 \\ 22 & 27 & 40 & 42 & 41 \\ 22 & 38 & 44 & 47 & 47\end{array}$

PV of debt-to-exports ratio

\section{Baseline}

A. Alternative Scenarios

A1. Key variables at their historical averages in 2009-2029 1/

2. New public sector loans on less favorable terms in 2009-2029 2

\section{B. Bound Test}

B1. Real GDP growth at historical average minus one standard deviation in 2010-2011

B2. Export value growth at historical average minus one standard deviation in 2010-2011 3/

B. US dollar GDP deflator at historical average minus one standard deviation in 2010-2011

4. Net non-debt creating flows at historical average minus one standard deviation in 2010-2011 4/

B5. Combination of Bl-B4 using one-half standard deviation shocks

B6. One-time 30 percent nominal depreciation relative to the baseline in $20105 /$

\section{Baseline}

\section{A. Alternative Scenarios}

A1. Key variables at their historical averages in 2009-2029 1/

A2. New public sector loans on less favorable terms in 2009-2029 2

\section{B. Bound Test}

B1. Real GDP growth at historical average minus one standard deviation in 2010-2011

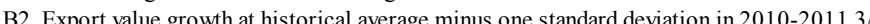

B3. US dollar GDP deflator at historical average minus one standard deviation in 2010-2011

B4. Net non-debt creating flows at historical average minus one standard deviation in 2010-2011

B5. Combination of B1-B4 using one-half standard deviation shocks

B6. One-time 30 percent nominal depreciation relative to the baseline in $20105 /$

$\begin{array}{rrrrrrrrrrrr}61 & 68 & 70 & 73 & 71 & 68 & 67 & 65 & 63 & 62 & \mathbf{6 0} & 43 \\ & & & & & & & & & & & \\ 61 & 41 & 30 & 25 & 19 & 16 & 14 & 12 & 10 & 8 & \mathbf{6} & 0 \\ 61 & 78 & 81 & 86 & 84 & 83 & 82 & 82 & 81 & 81 & \mathbf{8 0} & 68 \\ & & & & & & & & & & & \\ 61 & 63 & 63 & 65 & 61 & 57 & 54 & 52 & 49 & 47 & \mathbf{4 5} & 31 \\ 61 & 83 & 137 & 135 & 127 & 119 & 113 & 106 & 98 & 92 & \mathbf{8 6} & 46 \\ 61 & 63 & 63 & 65 & 61 & 57 & 54 & 52 & 49 & 47 & \mathbf{4 5} & 31 \\ 61 & 91 & 112 & 110 & 104 & 97 & 91 & 85 & 79 & 73 & \mathbf{6 8} & 34 \\ 61 & 76 & 112 & 111 & 105 & 98 & 93 & 87 & 81 & 76 & \mathbf{7 2} & 39 \\ 61 & 63 & 63 & 65 & 61 & 57 & 54 & 52 & 49 & 47 & \mathbf{4 5} & 31\end{array}$

\section{PV of debt-to-revenue ratio}

$\begin{array}{rrrrrrrrrrrr}60 & 78 & 90 & 98 & 94 & 92 & 91 & 90 & 89 & 87 & \mathbf{8 5} & 64 \\ & & & & & & & & & & & \\ 60 & 47 & 38 & 33 & 26 & 22 & 20 & 17 & 14 & 11 & \mathbf{8} & 0 \\ 60 & 89 & 104 & 114 & 112 & 111 & 112 & 113 & 114 & 113 & \mathbf{1 1 3} & 101 \\ & & & & & & & & & & & \\ 60 & 73 & 82 & 87 & 82 & 78 & 76 & 73 & 70 & 67 & \mathbf{6 5} & 47 \\ 60 & 84 & 127 & 129 & 121 & 116 & 111 & 105 & 99 & 93 & \mathbf{8 8} & 49 \\ 60 & 73 & 88 & 93 & 88 & 84 & 81 & 77 & 75 & 72 & \mathbf{6 9} & 50 \\ 60 & 104 & 144 & 146 & 137 & 131 & 125 & 117 & 110 & 103 & \mathbf{9 6} & 50 \\ 60 & 76 & 110 & 112 & 106 & 101 & 97 & 91 & 86 & 81 & \mathbf{7 7} & 44 \\ 60 & 107 & 119 & 126 & 119 & 114 & 109 & 105 & 101 & 97 & \mathbf{9 4} & 67\end{array}$


Table 10A.Moldova: Sensitivity Analysis for Key Indicators of Public and Publicly Guaranteed External Debt with Additional Non-Concessional Borrowing and no Impact on Growth, $2009-2029$ (continued) (In percent)

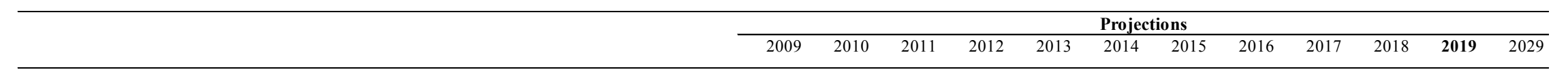

Debt service-to-exports ratio

\section{Baseline}

\section{A. Alternative Scenarios}

A1. Key variables at their historical averages in 2009-2029 1/

A2. New public sector loans on less favorable terms in 2009-2029 2

\section{B. Bound Tests}

B1. Real GDP growth at historical average minus one standard deviation in 2010-2011

B2. Export value growth at historical average minus one standard deviation in 2010-2011 3/

B3. US dollar GDP deflator at historical average minus one standard deviation in 2010-2011

B4. Net non-debt creating flows at historical average minus one standard deviation in 2010-2011 4 /

B5. Combination of B1-B4 using one-half standard deviation shocks

B6. One-time 30 percent nominal depreciation relative to the baseline in 20105 /

\section{Baseline}

\section{A. Alternative Scenarios}

A1. Key variables at their historical averages in 2009-2029 1/

A2. New public sector loans on less favorable terms in 2009-2029 2

\section{B. Bound Tests}

B1. Real GDP growth at historical average minus one standard deviation in 2010-2011

B2. Export value growth at historical average minus one standard deviation in 2010-2011 3/

B3. US dollar GDP deflator at historical average minus one standard deviation in 2010-2011

B4. Net non-debt creating flows at historical average minus one standard deviation in 2010-2011

B5. Combination of B1-B4 using one-half standard deviation shocks

B6. One-time 30 percent nominal depreciation relative to the baseline in 20105 /

Memorandum item

Grant element assumed on residual financing (i.e., financing required above baseline) 6

\section{Debt service-to-revenue ratio}

$\begin{array}{rrrrrrrrrrrrr}5 & 4 & 3 & 4 & 4 & 4 & 4 & 6 & 6 & 7 & \mathbf{7} & 6 \\ & & & & & & & & & & & \\ 5 & 3 & 2 & 2 & 2 & 2 & 1 & 1 & 1 & 1 & \mathbf{1} & 0 \\ 5 & 4 & 4 & 5 & 5 & 5 & 5 & 7 & 7 & 7 & \mathbf{7} & 8 \\ & & & & & & & & & & & \\ 5 & 4 & 3 & 4 & 4 & 4 & 4 & 5 & 5 & 6 & \mathbf{6} & 4 \\ 5 & 5 & 5 & 7 & 7 & 6 & 7 & 11 & 11 & 11 & \mathbf{1 1} & 6 \\ 5 & 4 & 3 & 4 & 4 & 4 & 4 & 5 & 5 & 6 & \mathbf{6} & 4 \\ 5 & 4 & 4 & 5 & 5 & 5 & 6 & 9 & 9 & 9 & \mathbf{9} & 5 \\ 5 & 4 & 4 & 5 & 6 & 5 & 6 & 9 & 9 & 9 & \mathbf{9} & 5 \\ 5 & 4 & 3 & 4 & 4 & 4 & 4 & 5 & 5 & 6 & \mathbf{6} & 4\end{array}$

Sources: Country authorities; and staff estimates and projections.

1/ Variables include real GDP growth, growth of GDP deflator (in U.S. dollar terms), non-interest current account in percent of GDP, and non-debt creating flows.

2/ Assumes that the interest rate on new borrowing is by 2 percentage points higher than in the baseline., while grace and maturity periods are the same as in the baseline.

3/ Exports values are assumed to remain permanently at the lower level, but the current account as a share of GDP is assumed to return to its baseline level after the shock (implicitly assuming

an offsetting adjustment in import levels).

4/ Includes official and private transfers and FDI.

5/ Depreciation is defined as percentage decline in dollar/local currency rate, such that it never exceeds 100 percent.

6/ Applies to all stress scenarios except for A2 (less favorable financing) in which the terms on all new financing are as specified in footnote 2. 
Table 11A.Moldova: Public Sector Debt Sustainability Framework, Baseline Scenario with Additional Non-Concessional Borrowing and no Impact on Growth, 2009-2029 (n percent of GDP, unless otherwise indicated)

\begin{tabular}{|c|c|c|c|c|c|c|c|c|c|c|c|c|c|c|c|}
\hline & & Actual & & & & Estimate & & & & & Projection & & & & \\
\hline & 2006 & 2007 & 2008 & Average & $\begin{array}{l}\text { Standard } \\
\text { Deviation }\end{array}$ & 2009 & 2010 & 2011 & 2012 & 2013 & 2014 & $\begin{array}{l}2009-14 \\
\text { Average }\end{array}$ & 2019 & 2029 & $\begin{array}{l}2015-29 \\
\text { Average }\end{array}$ \\
\hline $\begin{array}{l}\text { Public sector debt } 1 / \\
\text { o/w foreign-currency denominated }\end{array}$ & $\begin{array}{l}34.0 \\
25.6\end{array}$ & $\begin{array}{l}26.8 \\
19.8\end{array}$ & $\begin{array}{l}21.3 \\
15.7\end{array}$ & & & $\begin{array}{l}30.9 \\
22.4\end{array}$ & $\begin{array}{l}39.2 \\
30.9\end{array}$ & $\begin{array}{l}44.2 \\
36.8\end{array}$ & $\begin{array}{l}47.5 \\
41.0\end{array}$ & $\begin{array}{l}46.7 \\
41.1\end{array}$ & $\begin{array}{l}45.4 \\
40.5\end{array}$ & & $\begin{array}{l}41.3 \\
37.3\end{array}$ & $\begin{array}{l}28.3 \\
25.9\end{array}$ & \\
\hline Change in public sector debt & -3.6 & -7.2 & -5.5 & & & 9.6 & 8.2 & 5.0 & 3.3 & -0.7 & -1.3 & & -1.1 & -1.0 & \\
\hline Identified debt-creating flows & -6.0 & -8.5 & -6.3 & & & 12.9 & 5.1 & 2.2 & -0.6 & -2.8 & -2.6 & & -1.6 & -0.8 & \\
\hline Primary deficit & -1.3 & -1.0 & -0.2 & -2.0 & 3.5 & 7.5 & 5.7 & 3.5 & 1.6 & 0.1 & -0.4 & 3.0 & -0.5 & -0.3 & -0.4 \\
\hline Revenue and grants & 39.9 & 41.7 & 40.6 & & & 37.8 & 39.0 & 39.2 & 39.3 & 40.8 & 41.0 & & 41.0 & 41.0 & \\
\hline of which: grants & 0.7 & 1.8 & 1.7 & & & 1.9 & 3.2 & 2.5 & 1.8 & 1.7 & 1.6 & & 1.6 & 1.6 & \\
\hline Primary (noninterest) expenditure & 38.6 & 40.8 & 40.4 & & & 45.3 & 44.7 & 42.7 & 40.8 & 40.8 & 40.5 & & 40.5 & 40.6 & \\
\hline Automatic debt dynamics & -4.3 & -7.3 & -4.2 & & & 5.8 & 0.0 & -0.7 & -1.6 & -2.2 & -2.0 & & -1.2 & -0.5 & \\
\hline Contribution from interest rate/growth differential & -3.7 & -6.0 & -5.3 & & & 3.9 & 1.8 & -0.5 & -1.6 & -1.9 & -2.0 & & -1.2 & -0.5 & \\
\hline of which: contribution from average real interest rate & -2.0 & -5.0 & -3.4 & & & 1.7 & 2.3 & 0.7 & 0.5 & 0.3 & 0.2 & & 0.5 & 0.6 & \\
\hline of which: contribution from real GDP growth & -1.7 & -1.0 & -1.9 & & & 2.1 & -0.5 & -1.1 & -2.1 & -2.3 & -2.2 & & -1.6 & -1.1 & \\
\hline Contribution from real exchange rate depreciation & -0.6 & -1.3 & 1.1 & & & 2.0 & -1.9 & -0.2 & 0.1 & -0.3 & 0.0 & & $\ldots$ & $\ldots$ & \\
\hline Other identified debt-creating flows & -0.4 & -0.3 & -1.9 & & & -0.4 & -0.6 & -0.6 & -0.6 & -0.7 & -0.1 & & 0.0 & 0.0 & \\
\hline Privatization receipts (negative) & -0.4 & -0.3 & -1.9 & & & -0.4 & -0.6 & -0.6 & -0.6 & -0.7 & -0.1 & & 0.0 & 0.0 & \\
\hline Recognition of implicit or contingent liabilities & 0.0 & 0.0 & 0.0 & & & 0.0 & 0.0 & 0.0 & 0.0 & 0.0 & 0.0 & & 0.0 & 0.0 & \\
\hline Debt relief (HIPC and other) & 0.0 & 0.0 & 0.0 & & & 0.0 & 0.0 & 0.0 & 0.0 & 0.0 & 0.0 & & 0.0 & 0.0 & \\
\hline Other (specify, e.g. bank recapitalization) & 0.0 & 0.0 & 0.0 & & & 0.0 & 0.0 & 0.0 & 0.0 & 0.0 & 0.0 & & 0.0 & 0.0 & \\
\hline Residual, including asset changes & 2.3 & 1.3 & 0.8 & & & -3.3 & 3.1 & 2.8 & 3.9 & 2.1 & 1.3 & & 0.5 & -0.1 & \\
\hline Other Sustainability Indicators & & & & & & & & & & & & & & & \\
\hline PV of public sector debt & 8.5 & 7.0 & 20.5 & & & 30.2 & 36.3 & 40.4 & 43.0 & 42.3 & 41.2 & & 37.5 & 27.5 & \\
\hline $\mathrm{o} / \mathrm{w}$ foreign-currency denominated & 0.0 & 0.0 & 14.9 & & & 21.6 & 28.1 & 33.0 & 36.5 & 36.7 & 36.3 & & 33.5 & 25.1 & \\
\hline o/w external & $\ldots$ & $\ldots$ & 14.9 & & & 21.6 & 28.1 & 33.0 & 36.5 & 36.7 & 36.3 & & 33.5 & 25.1 & \\
\hline PV of contingent liabilities (not included in public sector debt) & $\ldots$ & $\ldots$ & $\ldots$ & & & $\ldots$ & & & $\ldots$ & $\ldots$ & $\ldots$ & & & & \\
\hline Gross financing need $2 /$ & 6.9 & 4.8 & 4.9 & & & 12.9 & 15.2 & 13.2 & 10.7 & 8.7 & 7.4 & & 7.6 & 5.6 & \\
\hline $\mathrm{PV}$ of public sector debt-to-revenue and grants ratio (in percent) & 21.2 & 16.8 & 50.4 & & & 79.9 & 93.0 & 103.0 & 109.4 & 103.9 & 100.6 & & 91.5 & 67.1 & \\
\hline $\mathrm{PV}$ of public sector debt-to-revenue ratio (in percent) & 21.6 & 17.6 & 52.7 & & & 84.1 & 101.3 & 109.9 & 114.7 & 108.3 & 104.5 & & 95.2 & 69.8 & \\
\hline o/w external 3/ & & & 38.3 & & & 60.2 & 78.2 & 89.8 & 97.5 & 93.9 & 92.0 & & 85.1 & 63.6 & \\
\hline Debt service-to-revenue and grants ratio (in percent) $4 /$ & 9.2 & 6.9 & 6.0 & & & 7.2 & 6.1 & 7.1 & 7.3 & 7.1 & 6.9 & & 10.6 & 9.0 & \\
\hline Debt service-to-revenue ratio (in percent) $4 /$ & 9.3 & 7.2 & 6.3 & & & 7.6 & 6.7 & 7.6 & 7.6 & 7.4 & 7.2 & & 11.0 & 9.3 & \\
\hline Primary deficit that stabilizes the debt-to-GDP ratio & 2.3 & 6.2 & 5.3 & & & -2.1 & -2.5 & -1.5 & -1.7 & 0.8 & 0.8 & & 0.6 & 0.6 & \\
\hline Key macroeconomic and fiscal assumptions & & & & & & & & & & & & & & & \\
\hline Real GDP growth (in percent) & 4.8 & 3.0 & 7.8 & 5.0 & 3.6 & -9.0 & 1.5 & 3.0 & 5.0 & 5.0 & 5.0 & 1.8 & 4.0 & 4.0 & 4.0 \\
\hline Average nominal interest rate on forex debt (in percent) & 3.3 & 1.9 & 1.8 & 3.3 & 1.1 & 2.3 & 2.5 & 2.2 & 2.2 & 2.1 & 2.1 & 2.2 & 2.4 & $3.4^{7}$ & 2.7 \\
\hline Average real interest rate on domestic debt (in percent) & -6.6 & -3.8 & 5.4 & -0.5 & 4.8 & 13.8 & 2.6 & 6.1 & 5.5 & 5.5 & $5.8^{\prime}$ & 6.5 & 7.6 & $10.0^{\circ}$ & 8.3 \\
\hline Real exchange rate depreciation (in percent,+ indicates depreciation) & -2.4 & -6.5 & 7.6 & -3.7 & 10.5 & 10.8 & & & $\ldots$ & $\ldots$ & $\ldots$ & $\ldots$ & $\ldots$ & $\ldots$ & \\
\hline Inflation rate (GDP deflator, in percent) & 13.4 & 15.9 & 9.2 & 16.0 & 10.1 & 3.9 & 6.5 & 4.7 & 4.7 & 4.3 & 4.0 & 4.7 & 4.0 & 4.0 & 4.0 \\
\hline Growth of real primary spending (deflated by GDP deflator, in percent) & 0.1 & 0.1 & 0.1 & 0.1 & 0.2 & 0.0 & 0.0 & 0.0 & 0.0 & 0.1 & 0.0 & 0.0 & 0.0 & 0.0 & 0.0 \\
\hline Grant element of new external borrowing (in percent) & $\ldots$ & $\ldots$ & $\ldots$ & $\ldots$ & $\ldots$ & -1.1 & 23.1 & 18.1 & 18.1 & 18.0 & 19.2 & 15.9 & 11.5 & -2.4 & $\ldots$ \\
\hline
\end{tabular}

Sources: Country authorities; and staff estimates and projections.
$1 /$ General government gross debt, excluding debt of state-owned enterprises.

1/ General government gross debt, excluding debt of state-owned enterprises.
2/ Gross financing need is defined as the primary deficit plus debt service plus the stock of short-term debt at the end of the last period.

$2 /$ Gross financing need is

$4 /$ Debt service is defined as the sum of interest and amortization of medium and long-term debt.

$5 /$ Historical averages and standard deviations are generally derived over the past 10 years, subject to data availability. 
Table 12A.Moldova: Sensitivity Analysis for Key Indicators of Public Debt with Additional Non-Concessional Borrowing and no Impact on Growth, 20092029

Projections

$\begin{array}{llllllll}2009 & 2010 & 2011 & 2012 & 2013 & 2014 & 2019 & 2029\end{array}$

PV of Debt-to-GDP Ratio

\section{Baseline}

\section{A. Alternative scenarios}

A1. Real GDP growth and primary balance are at historical averages A2. Primary balance is unchanged from 2009

A3. Permanently lower GDP growth $1 /$

\section{B. Bound tests}

B1. Real GDP growth is at historical average minus one standard deviations in 2010-201

B2. Primary balance is at historical average minus one standard deviations in 2010-2011

B3. Combination of B1-B2 using one half standard deviation shocks

B4. One-time 30 percent real depreciation in 2010

B5. 10 percent of GDP increase in other debt-creating flows in 2010

$\begin{array}{rrrrrrrr}30 & 36 & 40 & 43 & 42 & 41 & 37 & 28 \\ & & & & & & & \\ 30 & 28 & 26 & 25 & 23 & 21 & 11 & 0 \\ 30 & 38 & 46 & 55 & 61 & 67 & 100 & 152 \\ 30 & 37 & 42 & 46 & 46 & 47 & 56 & 93\end{array}$

$\begin{array}{llllllll}30 & 36 & 42 & 45 & 45 & 44 & 43 & 38\end{array}$

$\begin{array}{llllllll}30 & 32 & 34 & 37 & 37 & 36 & 32 & 23\end{array}$

$\begin{array}{llllllll}30 & 30 & 30 & 32 & 31 & 30 & 24 & 10\end{array}$

$\begin{array}{llllllll}30 & 45 & 49 & 51 & 50 & 49 & 47 & 44\end{array}$

$\begin{array}{llllllll}30 & 46 & 50 & 53 & 52 & 50 & 46 & 34\end{array}$

$\begin{array}{llllllll}80 & 93 & 103 & 109 & 104 & 101 & 92 & 67\end{array}$

Baseline

PV of Debt-to-Revenue Ratio 2/

\section{A. Alternative scenarios}

A1. Real GDP growth and primary balance are at historical averages

A2. Primary balance is unchanged from 2009

A3. Permanently lower GDP growth $1 /$

,

B. Bound tests

B1. Real GDP growth is at historical average minus one standard deviations in 2010-2011

B2. Primary balance is at historical average minus one standard deviations in 2010-2011

B3. Combination of B1-B2 using one half standard deviation shocks

B4. One-time 30 percent real depreciation in 2010

B5. 10 percent of GDP increase in other debt-creating flows in 2010

Debt Service-to-Revenue Ratio 2

\section{Baseline}

\section{A. Alternative scenarios}

A1. Real GDP growth and primary balance are at historical averages

A2. Primary balance is unchanged from 2009

A3. Permanently lower GDP growth 1/

$\begin{array}{rrrrrrrr}7 & 6 & 6 & 5 & 5 & 5 & 5 & 0 \\ 7 & 6 & 7 & 8 & 8 & 9 & 19 & 36 \\ 7 & 6 & 7 & 8 & 7 & 7 & 13 & 22\end{array}$

\section{B. Bound tests}

B1. Real GDP growth is at historical average minus one standard deviations in 2010-2011

B2. Primary balance is at historical average minus one standard deviations in 2010-2011

B3. Combination of B1-B2 using one half standard deviation shocks

B4. One-time 30 percent real depreciation in 2010

B5. 10 percent of GDP increase in other debt-creating flows in 2010

Sources: Country authorities; and staff estimates and projections.

1/ Assumes that real GDP growth is at baseline minus one standard deviation divided by the square root of the length of the projection period.

$2 /$ Revenues are defined inclusive of grants. 


\section{Statement by the IMF Staff Representative on the Republic of Moldova January 29, 2010}

21. This statement provides additional information on the implementation of the prior actions and presents new data on economic developments in Moldova that became available since the issuance of the staff report. The additional information does not change the thrust of the staff appraisal.

22. All prior actions for the Board consideration of the program have been carried out (MEFP Table 3). In December Parliament passed a 2010 budget in line with the Memorandum of Economic and Financial Policies (MEFP). Parliament also amended the 2009 Budget and the law on wages of budgetary sector employees in line with the MEFP. The National Bank of Moldova raised its net international reserves to US\$1341 million as of end-2009. Finally, Parliament transferred the authority for setting heating tariffs to the independent energy regulator (ANRE), which subsequently increased the tariffs by 29 percent, thus covering amortization and all variable costs.

23. The latest available data are broadly in line with staff's projections. The economy registered a $73 / 4$ percent year-on-year GDP decline in the third quarter of 2009, essentially unchanged from the January-June average, suggesting that output decline for the full 2009 could be limited to $8-8 \frac{1}{2}$ percent. End-2009 CPI inflation came at 0.4 percent, owing mainly to slower than anticipated effect of exchange rate depreciation on consumer prices. Inflation is however expected to pick up pace in the first quarter of 2010. The current account deficit in Q3 was 8.3 percent of period GDP, with some pick up in workers' remittances in the third quarter likely to limit the current account deficit for the full year to slightly below staff's projection of 8.9 percent of GDP. The fiscal data for November confirmed that the 2009 budget remained on track to keep the overall deficit below 9 percent of GDP. The banking system's capital adequacy ratio remained virtually unchanged in December at 32.3 percent, while the NPL ratio declined slightly to 16.3 percent.

24. The authorities have consented to the publication of the staff report, the MEFP, and the TMU for 2010-12. 
Press Release No.10/21

FOR IMMEDIATE RELEASE

January 29, 2010
International Monetary Fund

Washington, D.C. 20431 USA

\section{IMF Executive Board Approves US\$574 million ECF/EFF Arrangements for Moldova}

The Executive Board of the International Monetary Fund (IMF) today approved three-year arrangements for the Republic of Moldova under the Extended Credit Facility and the Extended Fund Facility. 16 With each facility providing an equal amount, the combined financial assistance will be equivalent to SDR 369.6 million (about US\$574.4 million) to support the country's economic program aimed at restoring fiscal and external sustainability, preserving financial stability, reducing poverty, and raising growth.

The approval makes an amount equivalent to SDR 60 million (about US\$93.2 million) immediately available, with the remainder available in installments subject to semiannual reviews.

The new arrangements follow a three-year program supported by a Poverty Reduction and Growth Facility, which was approved by the IMF Executive Board in May 2006 and expired in May 2009 (see Press Release No. 06/91).

${ }^{16}$ The Extended Credit Facility (ECF) has replaced the Poverty Reduction and Growth Facility (PRGF) as the Fund's main tool for medium-term financial support to low-income countries by providing a higher level of access to financing, more concessional terms, enhanced flexibility in program design features, and more focused streamlined conditionality. Financing under the ECF carries a zero interest rate, with a grace period of $5 \frac{1}{2}$ years, and a final maturity of 10 years. The Fund reviews the level of interest rates for all concessional facilities every two years. The Extended Fund Facility (EFF) carries an annual interest rate equal to the SDR basic rate of charge, and is repayable over 10 years with a 4.5 -year grace period on principal payments. 
Following the Executive Board discussion, Mr. Takatoshi Kato, Deputy Managing Director and Acting Chairman, said:

"The global economic crisis led to a rapid deterioration in the Moldovan economy in 2009. Falling demand in trading partners caused a severe downturn in exports and workers' remittances; FDI and other capital inflows fell dramatically as well. As a result, domestic demand collapsed, causing a sharp GDP contraction and deflationary pressures. Fiscal pressures were exacerbated by pre-election spending hikes.

“The authorities' program for 2010-12 aims to restore fiscal and external sustainability and boost growth. Fiscal policy targets a gradual return to a sustainable position by 2012, or earlier if possible. Monetary policy will focus on maintaining price stability. Structural reforms will support the recovery, including by increasing the flexibility of the highly regulated economy. The program will also increase spending for essential social services and poverty reduction.

"Fiscal policy in 2010 seeks to balance a much-needed adjustment with large public investment and social spending needs. To reduce the deficit, the authorities have rescheduled unaffordable wage increases and rationalized spending on materials and subsidies. At the same time, the budget envisages a rise in targeted social assistance spending to help protect vulnerable households.

"Taking advantage of low inflation, monetary policy will be supportive of the nascent economic recovery. To ensure that the exchange rate is in line with fundamentals, intervention in the foreign exchange market will be limited to smoothing short-run fluctuations. The central bank is closely monitoring banks' financial soundness and stands ready to take preemptive action if needed.

"Structural reforms are designed to unlock the economy's growth potential and support the fiscal program. A wide-ranging program of liberalization and deregulation is aimed at stimulating competition and fostering private sector-led growth. To keep the social insurance system financially sustainable, early retirement privileges of the civil servants will be gradually phased out, and sick leave compensation will be revamped. The authorities will also address the large quasi-fiscal arrears in the heating sector."

\section{ANNEX}

\section{Recent Economic Developments}

The global economic crisis hit Moldova hard, leading to a sharp weakening of the economy. In the first half of 2009, falling demand in trading partners led to a severe downturn in exports and remittances. While GDP dropped by nearly 8 percent over the same period, 
domestic demand declined even faster, and the current account deficit contracted to about 11 percent of period GDP. At the same time, the balance of payments moved from a surplus to a large deficit as Foreign Direct Investments (FDI) and other capital inflows fell dramatically. Deflation pressures persisted for most of 2009.

\section{Program Summary}

The program aims to restore fiscal and external sustainability, preserve financial stability, and raise growth. To facilitate the adjustment, the program provides for adequate budget financing.

Specifically, program objectives include:

(i) reversing the structural fiscal deterioration that occurred in 2008-2009 while safeguarding funds for public investment and priority social spending;

(ii) keeping inflation under control while rebuilding foreign reserves to cushion the economy from external shocks;

(iii)ensuring financial stability by enabling early detection of problems and strengthening the framework for bank rehabilitation and resolution; and

(iv)raising the economy's potential through structural reforms.

The program will also help mobilize resources for successful implementation of the poverty reduction agenda. To promote poverty reduction, the program sets a floor on priority social spending. Moreover, social assistance spending will be increased by 36 percent in 2010 relative to 2009 to support vulnerable households. 
Republic of Moldova: Selected Economic Indicators, 2007-10 1/

\begin{tabular}{rrrrr}
\hline & 2007 & 2008 & 2009 & 2010 \\
\cline { 3 - 4 } & & Est. & Proj. \\
\hline
\end{tabular}

I. Real sector indicators

Gross domestic product

Real growth rate

Demand

Consumption

Gross fixed capital formation

Nominal GDP (billions of Moldovan lei)

Consumer price index (end of period)

Average monthly wage (Moldovan lei)

Saving-investment balance

Foreign saving

National saving

Gross investment

II. Fiscal indicators (general government)

Primary balance (cash)

Overall balance (cash)

Stock of general government debt

III. Financial indicators

Broad money (M3)

Velocity (GDP/end-period M3; ratio)

Reserve money

Credit to the economy

IV. External sector indicators

Current account balance

Current account balance (percent of GDP)

Remittances and compensation of employees (net)

Gross official reserves

Gross official reserves (months of imports)

Real effective exchange rate, end-year, change (percent)

External debt (percent of GDP)

Debt service (percent of exports of goods and services)
(Percent change, unless otherwise indicated)

$\begin{array}{rrrr}3.0 & 7.8 & -9.0 & 1.5 \\ 7.8 & 5.7 & -18.9 & 2.3 \\ 3.8 & 5.7 & -7.0 & -1.7 \\ 25.5 & 2.2 & -38.4 & 6.1 \\ 53.4 & 62.9 & 59.5 & 64.3 \\ 13.1 & 7.4 & 2.5 & 5.0 \\ 2,063 & 2,529 & 2,750 & \ldots \\ & \text { (Percent of GDP) } & \\ 16.5 & 17.3 & 8.9 & 10.2 \\ 17.6 & 16.7 & 13.1 & 14.5 \\ 34.1 & 34.0 & 22.0 & 24.7 \\ & & & \\ 1.0 & 0.2 & -7.6 & -5.7 \\ -0.2 & -1.0 & -9.0 & -7.0 \\ 26.8 & 21.3 & 30.9 & 36.9\end{array}$

(End of period percent change, unless otherwise indicated)

$\begin{array}{rrrr}39.8 & 15.9 & -0.2 & 9.3 \\ 2.0 & 2.0 & 1.9 & 1.9 \\ 46.4 & 22.0 & -6.3 & 7.4 \\ 51.7 & 20.3 & -7.1 & 10.7\end{array}$

(Millions of U.S. dollars, unless otherwise indicated)

$\begin{array}{rrrr}-726 & -1,049 & -478 & -518 \\ -16.5 & -17.3 & -8.9 & -10.2 \\ 1,419 & 1,796 & 1,135 & 1,237 \\ 1,334 & 1,672 & 1,456 & 1,695 \\ 2.8 & 5.5 & 4.4 & 4.5 \\ 16.0 & 23.3 & -19.7 & \ldots \\ 64.2 & 55.9 & 66.0 & 78.6 \\ 13.7 & 16.6 & 19.2 & 20.8\end{array}$

Sources: Moldovan authorities; and IMF staff estimates and projections.

1/ Data exclude Transnistria. 


\section{Statement by Age Bakker, Executive Director for the Republic of Moldova and Victor Yotzov, Advisor to the Executive Director January 29, 2010}

The Moldovan authorities appreciate the constructive dialogue with the Fund management and staff over the years. They attach great value to the close relationship with the Fund, whose advice continues to support the policy formation process in Moldova.

\section{Recent Developments and Outlook}

After several years of strong growth, in 2009 Moldova was severely hit by the global economic crisis. Moreover, the economic crisis overlapped with a political crisis causing a major contraction of the economy. GDP is estimated to have decreased by about 9 percent, compared to a close to 8 percent growth in 2008. This huge swing was mostly result of a weak demand in trading partners, which caused a severe downturn in exports and worker remittances. The BOP moved from a surplus to a sizable deficit as FDI and other capital inflows fell dramatically. Even though deflation pressures emerged and persisted for a good part of 2009, monetary policy remained rather tight as the central bank was defending the leu, thus tightening liquidity and depleting its foreign reserves. On top of that, the crisis and pre-election spending hikes brought about a large and widening fiscal gap as revenue dropped, notably the VAT and foreign trade taxes, while current fiscal expenditure was substantially increased in the run-up of the elections.

The financial system, dominated by the banking sector, remains stable, although the recession has negatively affected the quality of the credit portfolio in many banks. The significant decline in credit, along with capital increases in a number of banks, has brought the average risk-weighted capital adequacy ratio (CAR) of the system to nearly three-times the required minimum of 12 percent. However, the nonperforming loans are on the rise, even though their doubtful and loss components remain low. One medium-sized bank became insolvent in June, mostly because of a high portfolio concentration in recession-hit sectors, as well as some risk management irregularities. The National Bank of Moldova (NBM) has responded by stepping up supervision and regulation. Banks have been requested to undergo a diagnostic study performed by an independent reputable audit firm to assess the quality of their assets and review their risk management methods. The NBM has also initiated close and enhanced monitoring of bank activity on the basis of monthly bank reports on financial soundness indicators and stress tests.

With regard to 2010 , the macroeconomic objectives are influenced by the expected gradual recovery in Moldova's trading partners. The authorities expect the recession to be over by the first quarter of 2010, allowing the annual growth rate to rebound to 1.5 percent. Monetary policy is aimed to achieve (and sustain over the next few years) inflation around 5 percent. The current account deficit is expected to widen modestly to slightly above 10 percent of GDP as imports are boosted by higher prices of imported energy and a recovery in investment.

Over the medium term, the economy is expected to recover to its potential, with growth reaching about 5 percent by 2012. Inflation is projected to remain stable in mid-single digits, anchored by growing credibility and transparency of monetary policy. Remittances will grow slowly back to their 2008 level over the medium term. The current account deficit is expected 
to stabilize around 10 percent of GDP - a level that should be readily financed by FDI and official assistance, which would also help reserves grow to adequate levels.

\section{Program Objectives}

While it could be argued that the economic crisis was triggered by an external shock, the new government acknowledges that the policies ran in the first half of 2009 were unsustainable and led to a sharp deterioration of the economy's fiscal and external position. The stated overarching objective of the authorities is to improve the well-being of the population by promoting sustainable growth and reducing poverty. At the same time, the authorities realize that in the current juncture they need to undertake urgent measures to stabilize and rebalance the economy. To this end, the authorities request Fund-supported arrangements to restore fiscal and external sustainability, preserve financial stability, and support growth. In addition, the authorities are committed to wide and far-reaching structural reforms to raise the economy's potential.

The economic crisis, exacerbated by a political turmoil following the April elections, led to delays in implementation of market-oriented reforms and a properly targeted social safety net for the poor. A number of sectors of the national economy are still excessively regulated with high barriers to entry and low competition. In response to the economic crisis, the new government launched an Economic Stabilization and Recovery Plan, which puts forward strong measures intended to help enterprises and households overcome the economic crisis.

The measures envisaged in the plan, a large part of which are included in the MEFP, aim to stabilize public finances, restart economic growth, and protect vulnerable households. In particular, to create a modern European public administration, a series of reforms have been launched to streamline and enhance the efficiency of the civil service. To provide a boost to trade and promote competition, the authorities have removed many formal and informal export and import restrictions.

\section{Fiscal Policy}

The Moldovan authorities are firmly committed to a prudent and transparent fiscal policy. They are fully aware that this is a challenging task given that the program should seek to balance the needed sizable fiscal adjustment with large public investment and social spending needs. In this regard, the authorities are struggling to avoid any further build-up of fiscal pressures, while ensuring smooth execution of the budget so to prevent distortion of the economic activity. In order to quickly fix some of the problems, the new government adopted a number of policy decisions to reduce the budget deficit for 2009 as much as feasible. A first step in this direction was postponing wage increases for employees of the budgetary sector as envisaged in the Budget System Wage Law.

Realizing that the share of public sector employment (over 20 percent) is too high even compared to higher-income emerging market countries, the new government decided to stick to a previous action plan aimed at optimizing the number of employees in the budgetary sector. This plan envisages reducing staff by about 10 thousand people in the period 2008-10.

Another important objective of the authorities' program is to reduce spending on goods and services and to cut low-priority subsidies to enterprises. On the revenue side, the government has raised excises on a number of products (including gasoline, diesel, tobacco products, 
liquor, cosmetics, luxury cars, etc.) and raised the VAT rate on natural gas. In addition, the corporate income tax for reinvested profits, which in 2008 was set at a zero rate, will be reinstated with a low single rate (10 percent) and a broad, uniform base across sectors and regions. In order not to raise the tax burden on companies before the economy recovers from the crisis, this measure will apply to income earned from 2012 onwards.

To ensure appropriate budget financing in 2010, the authorities request that a moderate fraction of the access under the Fund arrangements be used for direct budget support. Moldova lacks access to international markets, and the domestic market for government securities has limited capacity, as experience in mid-2009 showed. The absence of Fund financing would imply either additional sharp expenditure cuts, detrimental to the economic recovery or the program's social assistance policies, or accumulation of arrears.

\section{Monetary and Exchange Rate Policy}

The authorities' intention is to leave monetary policy relaxed as long as the inflation outlook is in line with the NBM's target. In that sense, there is scope for further easing in the short run. Concomitantly, the NBM has approved a new strategy upholding price stability as the primary objective of monetary policy and the NBM's base rate as the main policy instrument. The NBM will restart open market operations, work toward enhancing its forecasting and analytical capacity, and promote transparency through regular communication with the public regarding its inflation forecast and policy stance. Also, the NBM plans to start publishing quarterly reports providing analyses of monetary policy performance as well as forecasts of inflation and key macroeconomic indicators.

Within its new framework, the NBM is committed to promote a flexible exchange rate with interventions in the foreign exchange market limited to only preventing excessive fluctuations without resisting trends. In addition, the NBM is preparing to take advantage of the easing of global financial tensions and to replenish its foreign exchange reserves to a more appropriate level. More specifically, the goal is to build a comfortable buffer against external shocks in the range of 70-90 percent of next year's current account deficit and maturing external debt. This coverage is believed to be appropriate given the still high downside risks to external stability.

\section{Structural Reform}

To better direct its limited resources to the most vulnerable households, the new government is determined to give priority to a targeted compensation system, based on the households' income and assets. Currently, the social assistance system covers only a quarter of the vulnerable households and any abrupt removal of nominal compensations would produce undesirable social effects. The authorities realize that for the new system to become operational, a series of concrete steps will need to be taken. In particular, the authorities will terminate, as of the beginning of 2010, granting the right to nominal compensation; freezing the nominal amount of compensations granted; and gradual elimination of nominal compensations categories. In the second stage, by end-March 2010, the government will approve a plan for a prompt expansion of the new targeted social assistance system with a view to cover at least $2 / 3$ of all eligible recipients by end-2010. An intensive communication 
campaign is already underway to promote enrollment of eligible households into the new system.

The government attaches high priority to eliminating quasi-fiscal expenditure. In this regard, it's the authorities' understanding that heating tariffs should be kept away from the political debate and be based on cost-recovery levels. Accordingly, the current legislation has been amended to ensure de-politicization of the tariff-setting system and strengthening of the role of the independent National Agency for Energy Regulation (ANRE).

Longer-term sustainability of the pension system is another area that needs reforms. The ultimate goal is a smooth transition from the current pay-as-you-go system to a contributionbased system. To this end, the authorities are preparing concrete measures, including phasing out early retirement options for certain professional categories; extending the requirement to pay state social insurance contributions to all persons employed in Moldova; and improving the mechanism for granting the right to compensation for temporary disability. These measures aim at strengthening of the first pillar of the future pension system. With regard to the implementation of the second pillar, the authorities recognize that it will involve substantial financial costs, which will need to be viewed in the context of wider financial and technical assistance from development partners.

There are numerous measures that the authorities are already undertaking to simplify business regulations and improve the business climate. Amongst the most important are: revising the classification of regulated goods that are subject to mandatory compliance certification; implementation of a true one-stop shop for business registration; and improving the access to financing through institutionalization of an operational system for storing and disseminating credit information. The authorities strongly believe that the successful implementation of these reforms will be crucial for jump-starting the economic recovery.

Finally, the Moldovan authorities believe that the policies set forth in the MEFP are well designed and sufficient to achieve the objectives of the program. At the same time, they stand ready to take any further measures that may become necessary. In accordance with the Fund's policy, the authorities will consult with the Fund on the adoption of these measures, and in advance of revisions to the policies contained in the MEFP. 